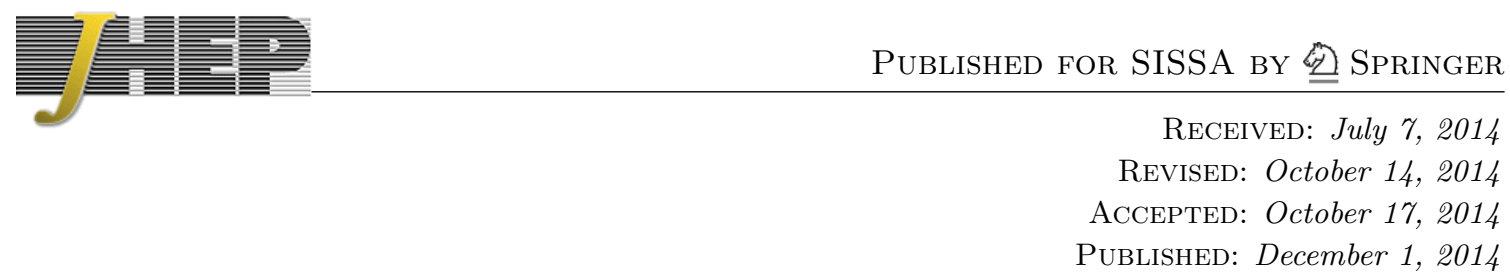

\title{
Massive nonplanar two-loop maximal unitarity
}

\section{Mads Søgaard and Yang Zhang}

Niels Bohr International Academy and Discovery Center, Niels Bohr Institute, University of Copenhagen, Blegdamsvej 17, DK-2100 Copenhagen, Denmark

E-mail: madss@nbi.dk, zhang@nbi.dk

ABSTRACT: We explore maximal unitarity for nonplanar two-loop integrals with up to four massive external legs. In this framework, the amplitude is reduced to a basis of master integrals whose coefficients are extracted from maximal cuts. The hepta-cut of the nonplanar double box defines a nodal algebraic curve associated with a multiply pinched genus-3 Riemann surface. All possible configurations of external masses are covered by two distinct topological pictures in which the curve decomposes into either six or eight Riemann spheres. The procedure relies on consistency equations based on vanishing of integrals of total derivatives and Levi-Civita contractions. Our analysis indicates that these constraints are governed by the global structure of the maximal cut. Lastly, we present an algorithm for computing generalized cuts of massive integrals with higher powers of propagators based on the Bezoutian matrix method.

KEYwords: Scattering Amplitudes, Differential and Algebraic Geometry

ARXIV EPRINT: 1406.5044 


\section{Contents}

1 Introduction 1

2 Maximal unitarity 3

2.1 Multivariate residues 4

3 Parametrization of hepta-cut solutions $\quad 6$

$\begin{array}{lll}3.1 & \text { Mutually projected kinematics } & 7\end{array}$

$\begin{array}{llr}3.2 & \text { Four-mass hepta-cut equations } & 9\end{array}$

$\begin{array}{lll}3.3 & \text { Massless limits } & 12\end{array}$

$\begin{array}{ll}3.4 & \text { Classification of kinematic solutions } \\ \end{array}$

4 Residues of the loop integrand and topological structure $\quad 15$

$\begin{array}{ll}4.1 \text { Hextuply pinched genus-3 curve } & 17\end{array}$

$\begin{array}{ll}4.2 & \text { Octuply pinched genus-3 curve } 20\end{array}$

5 Master integral projectors $\quad 21$

5.1 One-mass projectors 23

$\begin{array}{lll}5.2 & \text { Two-mass projectors } & 26\end{array}$

5.3 Three-mass projectors 30

$\begin{array}{lll}5.4 & \text { Four-mass projectors } & 32\end{array}$

6 Reduction of integrals with doubled propagators $\quad 34$

6.1 Unitarity cut algorithm: Bezoutian matrix method 34

6.2 Example: one-mass two-loop crossed box 39

7 Discussion and conclusion $\quad 42$

A Explicit parametrization of hepta-cut solutions $\quad 44$

A.1 The four-mass case and smooth massless limits 45

$\begin{array}{lll}\text { A.2 Degenerate massless limits } & 46\end{array}$

$\begin{array}{ll}\text { B Structure of global poles } & 47\end{array}$

\section{Introduction}

Contemporary experimental high energy physics is concentrated on the Large Hadron Collider (LHC) at CERN. Our ability to utilize the huge amount of data delivered by the experiment towards further scientific progress relies on a quantitative understanding of all relevant scattering processes in the Standard Model. Otherwise, we are unable to extract 
signals of new physics from the background. Precise theoretical predictions in Quantum Chromodynamics (QCD) at the LHC require not only amplitudes at leading order (LO) and next-to-leading order (NLO), but also next-to-next-to leading order (NNLO) corrections to comply with the level of accuracy of the data. For some processes, two-loop amplitudes are important already at NLO because the LO terms begin at one loop.

The text book approach to perturbative scattering amplitudes is through Feynman rules and diagrams. Although it tracks interactions of particles very intuitively and in principle always works, this method suffers from severe computational problems with increasing loop level or number of external legs. The main reason is that the gauge redundancy of the theory introduces virtual intermediate states that are off-shell. Not even very powerful computers are able to deal with many of the phenomenologically interesting processes without new clever ways to attack the problem.

The last two decades have seen many attempts to surmount the computational bottleneck. The lesson is to exploit analyticity and unitarity of the scattering matrix. Analyticity allows for amplitudes to be reconstructed from their singularity structure, whereas by unitarity, residues at the poles factorize onto products of simple amplitudes. Two of the most successful advances are the original unitarity method for loop amplitudes developed by Bern, Dixon, Dunbar and Kosower [1,2] and the Britto-Cachazo-Feng-Witten (BCFW) recursion relations $[3,4]$ for trees. In these works, striking and otherwise completely unexpected structure and simplicity are revealed by virtue of retaining only physical on-shell information in a Lagrangianless setting. In a nutshell, all trees may now be constructed recursively and further fused into loops.

The basic idea of the unitarity method (see also later studies, e.g. refs. [5-24]) is to reconstruct the amplitude from double cuts that place internal lines in a given channel on their mass-shell and break it into a product of trees. Many individual contributions share such minimal cuts and are therefore hard to separate. Therefore intermediate algebraic steps are typically needed. In that view, the generalized unitarity method [7, 18] is more efficient because several propagators are cut simultaneously and thus fewer integrals are isolated. Thanks to the unitarity method, otherwise unfeasible computations of $2 \rightarrow 2$ massless scattering processes in QCD have been carried out.

In the last couple of years, two-loop amplitudes have received substantial attention in the literature. The integrand-level reduction method of Ossola, Papadopoulos and Pittau (OPP) has been extended to multi loops using computational algebraic geometry, and a general way of classifying high-loop unitarity cut solutions is now available [47, 5465]. These techniques were used by Badger, Frellesvig and one of the present authors to calculate the planar part of the all-plus two-loop five-gluon amplitude in QCD [58] and also demonstrated for the planar triple box [56]. In ref. [66] the unitarity method was applied to two-loop diagrams to determine their integral bases.

Working directly at the level of the integral basis, the maximal unitarity formalism initiated by Kosower and Larsen in ref. [46] has emerged as an extension of the quadruple and triple cut at one loop [7, 18]. In maximal unitarity one expands the amplitude in a basis of integrals and seeks to isolate the integral coefficients by finding multidimensional complex integration contours that are uniquely associated with each individual master 
integral. One of the major advantages of maximal unitarity is that one may circumvent the integrand basis which is typically considerably larger than the integral basis. After the reduction onto master integrals is complete, each coefficient is extracted as a linear combination of residues of the product of trees that arise when the diagram falls apart on-shell. The tree-level data is easy to manage using superspace techniques [67,68]. The leading singularity method $[42,43]$ previously addressed hepta-cuts and octa-cuts at two loops in $\mathcal{N}=4$ super Yang-Mills theory.

Until now, maximal unitarity has remained relatively unexplored in the nonplanar sector [50]. In this paper, we extend the framework to two-loop crossed-box integrals with up to four external massive legs. Indeed, inspection of the nonplanar part of the integral basis for, e.g., $g g \rightarrow V_{1} V_{2}$ computed in ref. [69] shows that in practice the two-loop crossed boxes constitute most of the nonplanar basis. ${ }^{1}$ Remarkably, we find that essentially all features of maximal unitarity observed in the planar sector [46-49] carry over directly to the nonplanar sector. In particular, in determining projectors for the master integrals, we find that the global structure of the maximal cut seems to govern consistency equations from integration-by-parts (IBP) identities and the number of master integrals. Moreover, we show that the constraints are inherited through chiral branchings between distinct classes of hepta-cut solutions.

The paper is organized in the following way. In section 2 we review the maximal unitarity method and the theory of multivariate residues. In sections 3-5 we respectively parametrize the hepta-cut solutions using mutually projecting kinematics, analyze the global structure of the maximal cut and impose consistency relations in order to uniquely fix the projectors for the master integral coefficients for all kinematically inequivalent configurations. Finally, in section 6 , we present an enhanced algorithm to compute degenerate multivariate residues from generalized unitarity cuts and apply the technique to massive integrals with doubled propagators.

\section{Maximal unitarity}

The modern version of the unitarity method relies on the existence of a finite basis of linearly independent master integrals $\left\{I_{i}\right\}$ onto which the amplitude in consideration can be expanded, up to additional rational terms,

$$
\mathcal{A}_{n}^{L \text {-loop }}=\sum_{i \in \text { Basis }} c_{i} I_{i}+\text { rational terms . }
$$

Therefore, if the basis integrals are known explicitly in dimensional regularization, calculating an amplitude boils down to determining the rational coefficients $\left\{c_{i}\right\}$. The trick is to apply generalized unitarity cuts to either side of eq. (2.1),

$$
\Delta \mathcal{A}_{n}^{L-\text { loop }}=\sum_{i \in \text { Basis }} c_{i} \Delta I_{i}
$$

and exploit that the cut amplitude factorizes onto simpler quantities.

\footnotetext{
${ }^{1}$ Moreover, we have checked that the remaining crossed triangles also have leading singularities, and they are therefore expected to be amenable to the techniques used here.
} 
At the one-loop level, the basis consists of boxes, triangles and bubbles with scalar numerators only. Here the computation has already been fully automated, see refs. $[7,18$, 19, 25]. For instance, a box coefficient is isolated by a quadruple cut and thereby becomes the product of the trees at the four corners, evaluated in on-shell kinematics. The on-shell internal lines are complex valued for general external momenta. This implies that the cut prescription in terms of Dirac Delta functions necessarily must be reformulated by means of a multidimensional complex contour integral encircling global poles [46, 47].

At two loops and beyond, the situation is more intricate, one of the main reasons being that a minimal integral basis is not yet known. Integrals with numerator insertions are in general algebraically irreducible and the reduction to master integrals inevitably involves IBP identities. As a consequence, multiple contributions contaminate the unitarity cuts. Although maximal cuts for four particles at two and three loops have superficial resemblance to the quadruple cut at one loop, it is also challenging to extract the coefficients because the cut does not localize integrals to a point, but rather an algebraic curve [46, 47] or generally speaking, an algebraic surface [51, 64].

In the last couple of years, maximal unitarity has been applied to the planar double box with up to four external massive legs $[46,48,49]$ in general theories and to the massless twoloop crossed box [50]. Recently, the formalism was also extended to amplitude contributions whose maximal cuts define multidimensional algebraic varieties [51], exemplified for the planar triple box at three loops. Along these lines, the unitarity cut prescription has been extended to accommodate loop integrals with doubled or higher powers of propagators [52].

\subsection{Multivariate residues}

Inspired by the discussion in the introduction, we start by reviewing basic theory of multivariate residues, with emphasis on computation of nondegenerate residues. We also refer the reader to classical text books by Griffiths and Harris [71] and Hartshorne [72].

Let $U=\left\{z \in \mathbb{C}^{n}:\|z-\xi\|<\epsilon\right\}$ for $\epsilon>0$ be a small ball around $z=\xi$ and assume that $f$ and $h$ are holomorphic maps in a neighborhood of the closure $\bar{U}$ of $U$. For our purposes, it is in fact sufficient to think of each component of $f$ and $h$ as just being multivariate polynomials of certain degrees. Furthermore, suppose that $f^{-1}(0) \cap U=\{\xi\}$, i.e. the components of $f$ have exactly one simultaneous zero $\xi \in U$. Then for the meromorphic $n$-form,

$$
\omega=\frac{h(z) d z_{1} \wedge \cdots \wedge d z_{n}}{f_{1}(z) \cdots f_{n}(z)}
$$

the associated residue at $z=\xi$ is computed by an integral over a contour that is topologically equivalent to a torus of real dimension $n$ embedded in $\mathbb{C}^{n}$. In detail we have

$$
\operatorname{Res}_{\left\{f_{1}, \ldots, f_{n}\right\}, \xi}(\omega)=\frac{1}{(2 \pi i)^{n}} \oint_{\Gamma_{\epsilon}} \frac{h(z) d z_{1} \wedge \cdots \wedge d z_{n}}{f_{1}(z) \cdots f_{n}(z)}
$$

where $\Gamma_{\epsilon}=\left\{z \in \mathbb{C}^{n}:\left|f_{i}(z)\right|=\epsilon_{i}\right\}$.

We remark several elementary properties of the residue. The residue is linear in $h$, but alternating in the $f_{i}$ s. Moreover, the value of a residue is invariant under nonsingular 
complex coordinate transformations. It is not hard to prove by Stokes' theorem that if locally $h \in I_{f}=\left\langle f_{1}, \ldots, f_{n}\right\rangle$, where $I_{f}$ is the ideal generated by the $f_{i} \mathrm{~s}$, that is,

$$
h(z)=a_{1}(z) f_{1}(z)+\cdots+a_{n}(z) f_{n}(z),
$$

for holomorphic functions $a_{i}$ in a neighborhood of $\xi$, then we have

$$
\operatorname{Res}_{\left\{f_{1}, \ldots, f_{n}\right\}, \xi}(\omega)=0 \text {. }
$$

For the calculation of a residue we distinguish between three classes of increasing difficulty: factorizable, nondegenerate and degenerate residues. If each $f_{i}$ defines a univariate polynomial, i.e. $f_{i}(z)=f_{i}\left(z_{i}\right)$, the contour factorizes onto a product of univariate contours such that the residue can be obtained in a manner that trivially resembles the one-dimensional case,

$$
\operatorname{Res}_{\left\{f_{1}, \ldots, f_{n}\right\}, \xi}(\omega)=\frac{1}{(2 \pi i)^{n}} \oint_{\left|f_{1}\left(z_{1}\right)\right|=\epsilon_{1}} \frac{d z_{1}}{f_{1}\left(z_{1}\right)} \cdots \oint_{\left|f_{n}\left(z_{n}\right)\right|=\epsilon_{n}} \frac{d z_{n}}{f_{n}\left(z_{n}\right)} h(z)
$$

If the $f_{i}$ s are not univariate polynomials and the Jacobian determinant of $f_{1}, \ldots, f_{n}$ evaluated at $z=\xi$ is nonzero,

$$
\left.J(\xi) \equiv \operatorname{det}_{i, j}\left(\frac{\partial f_{i}}{\partial z_{j}}\right)\right|_{z=\xi} \neq 0
$$

the residue is said to be nondegenerate. In that case it is natural to define the residue via appropriate coordinate transformation as

$$
\operatorname{Res}\left\{f_{1}, \cdots, f_{n}\right\}, \xi(\omega)=h(\xi) / J(\xi) \text {. }
$$

We immediately recognize the localization property (2.9) as the obvious generalization of the Dirac delta function to several complex variables once we define

$$
\int\left[\prod_{k=1}^{n} d z_{k}\right] h(z) \prod_{j=1}^{n} \delta\left(z_{j}-\xi_{j}\right) \equiv \frac{1}{(2 \pi i)^{n}} \oint_{\Gamma_{\epsilon}(\xi)} d z_{1} \wedge \cdots \wedge d z_{n} \frac{h(z)}{\prod_{j=1}^{n}\left(z_{j}-\xi_{j}\right)}
$$

In particular, this observation allows us to define generalized unitarity cuts of amplitude contributions that only factorize for complex kinematics.

In general, a multivariate residue is neither nondegenerate nor factorizable and we then proceed by means of computational algebraic geometry and use the transformation law and Gröbner basis method as we will explain in section 6. Examples of multiloop unitarity cuts that give rise to degenerate multivariate residues include among others the three-loop planar triple box [51] and integrals with doubled or higher powers of propagators [52]. In the two-loop crossed-box computation we will mostly encounter nondegenerate residues. 


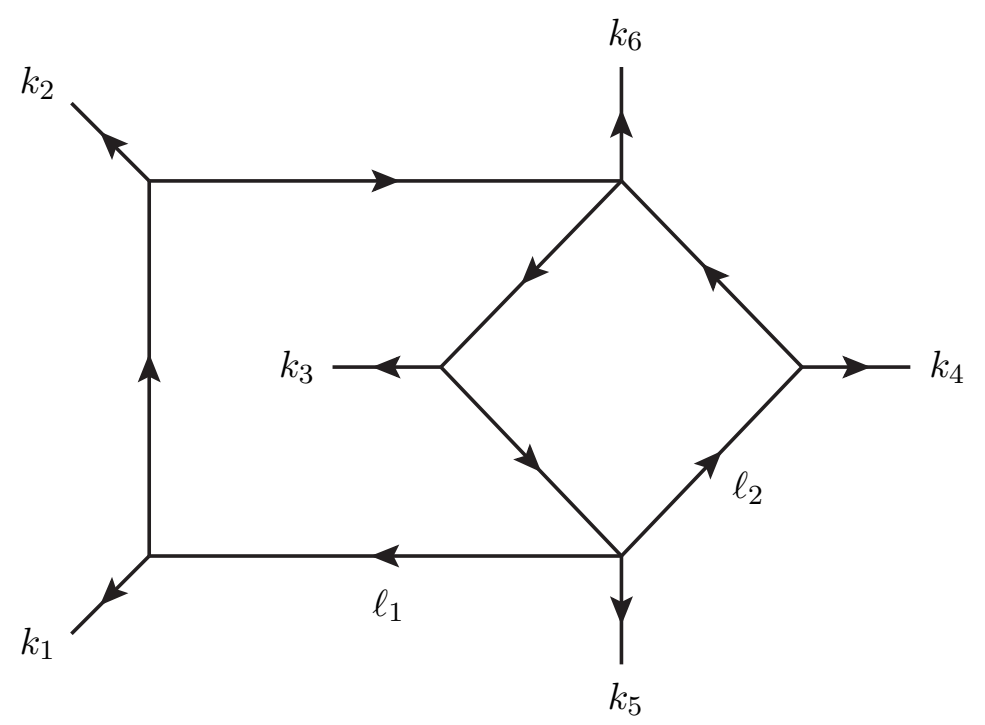

Figure 1. The two-loop crossed-box integral. All external particles may be massive.

\section{Parametrization of hepta-cut solutions}

The dimensionally regularized Feynman scalar integral for the two-loop crossed box amplitude contribution with possibly massive external legs $k_{1}, \ldots, k_{6}$ distributed across all six vertices is

$$
X_{2,1,1}[1]=\int_{\mathbb{R}^{D}} \frac{d^{D} \ell_{1}}{(2 \pi)^{D}} \int_{\mathbb{R}^{D}} \frac{d^{D} \ell_{2}}{(2 \pi)^{D}} \prod_{k=1}^{7} \frac{1}{f_{k}\left(\ell_{1}, \ell_{2}\right)}
$$

where the inverse propagators are

$$
\begin{array}{llll}
f_{1}=\ell_{1}^{2}, & f_{2}=\left(\ell_{1}-k_{1}\right)^{2}, & f_{3}=\left(\ell_{1}-k_{1}-k_{2}\right)^{2}, & \\
f_{4}=\ell_{2}^{2}, & f_{5}=\left(\ell_{2}-k_{4}\right)^{2}, & f_{6}=\left(\ell_{1}+\ell_{2}+k_{5}\right)^{2}, & f_{7}=\left(\ell_{1}+\ell_{2}+k_{3}+k_{5}\right)^{2} .
\end{array}
$$

Conventions and momentum flow are shown in figure 1. The Feynman $i \epsilon$-prescription has been suppressed as it is irrelevant for our purposes. Generally speaking, this integral will have a nontrivial polynomial numerator function denoted $\Phi\left(\ell_{1}, \ell_{2}\right)$ and is in that situation referred to as a tensor integral even though all Lorentz indices are properly contracted.

The seven inverse propagators $\left\{f_{i}\right\}$ generate a polynomial ideal $I=\left\langle f_{1}, \ldots, f_{7}\right\rangle$ and the hepta-cut equations define a complex algebraic curve (or two-dimensional real surface) which is the zero locus of $I$. In our notation,

$$
\begin{aligned}
\mathcal{S} \equiv \mathcal{Z}(I)=\left\{\left(\ell_{1}, \ell_{2}\right) \in\left(\mathbb{C}^{4}\right)^{\otimes 2} \mid\right. & \ell_{1}^{2}=0,\left(\ell_{1}-k_{1}\right)^{2}=0,\left(\ell_{1}-k_{1}-k_{2}\right)^{2}=0, \\
\ell_{2}^{2} & =0,\left(\ell_{2}-k_{4}\right)^{2}=0,\left(\ell_{1}+\ell_{2}+k_{5}\right)^{2}=0, \\
& \left.\left(\ell_{1}+\ell_{2}+k_{3}+k_{5}\right)^{2}=0\right\} .
\end{aligned}
$$


The curve is generally reducible and the algebraic set $\mathcal{S}$ can always be decomposed uniquely into a union of a finite number $n$ of irreducible components which are in one-to-one correspondence with the inequivalent hepta-cut solutions,

$$
\mathcal{S}=\bigcup_{i=1}^{n} \mathcal{S}_{i}, \quad \mathcal{S}_{i} \not \subset \mathcal{S}_{j} \text { if } i \neq j .
$$

The existence of such a decomposition can be proved by algebraic geometry and primary decomposition of the polynomial ideal, see ref. [57]. The number of solutions within an integral topology depends on the kinematic configuration, in particular the distribution of massive and massless legs.

In the rest of this paper, we examine four-dimensional amplitude contributions with two-loop crossed box topology with $k_{5}=k_{6}=0$, allowing any other configuration of massive and massless external legs. The Mandelstam invariants are throughout this paper defined as

$$
s_{12} \equiv\left(k_{1}+k_{2}\right)^{2}, \quad s_{13} \equiv\left(k_{1}+k_{3}\right)^{2}, \quad s_{14} \equiv\left(k_{1}+k_{4}\right)^{2},
$$

so that momentum conservation can be stated as

$$
s_{12}+s_{13}+s_{14}=m_{1}^{2}+m_{2}^{2}+m_{3}^{2}+m_{4}^{2} .
$$

The four-point massless two-loop crossed box was previously studied in terms of residues and multidimensional contour integrals by one of the present authors in ref. [50] and by integrand-level reduction by Badger, Frellesvig and the other author in ref. [54].

\subsection{Mutually projected kinematics}

Scattering amplitudes of massless particles are naturally encoded in the spinor helicity formalism by Lorentz invariant inner products of commuting spinors $\lambda_{i}^{\alpha}$ and $\tilde{\lambda}_{i}^{\dot{\alpha}}$. For a momentum $k_{i}$ with $k_{i}^{2}=0$ we have the representation $k_{i}^{\alpha \dot{\alpha}}=\lambda_{i}^{\alpha} \tilde{\lambda}_{i}^{\dot{\alpha}}$. It is then possible to define antisymmetric chiral and antichiral brackets,

$$
\langle i j\rangle=-\langle j i\rangle \equiv \epsilon_{\alpha \beta} \lambda_{i}^{\alpha} \lambda_{j}^{\beta}, \quad[i j]=-[j i] \equiv \epsilon_{\dot{\alpha} \dot{\beta}} \lambda_{i}^{\dot{\alpha}} \lambda_{j}^{\dot{\beta}},
$$

and make contact to the Mandelstam invariants,

$$
s_{i j}=\langle i j\rangle[j i]=2 k_{i} \cdot k_{j} .
$$

We treat the massive hepta-cut equations for the two-loop crossed box using mutually projected kinematics $[15,18]$. Thereby the spinor helicity formalism and massive momenta become compatible. Given a pair of massive momenta $\left(k_{i}, k_{j}\right)$, the idea is to obtain massless momenta $\left(k_{i}^{b}, k_{j}^{b}\right)$ each of which is the massless projection of one of the massive legs in the direction of the other masslessly projected leg. Here we will consider four-point kinematics with mutually projecting pairs $\left(k_{1}, k_{2}\right)$ and $\left(k_{3}, k_{4}\right)$. Other choices are of course also possible. Within each pair we define

$$
k_{j, 1}^{b, \mu}=k_{j, 1}^{\mu}-\frac{k_{j, 1}^{2}}{2 k_{j, 1} \cdot k_{j, 2}^{b}} k_{j, 2}^{b, \mu}, \quad k_{j, 2}^{b, \mu}=k_{j, 2}^{\mu}-\frac{k_{j, 2}^{2}}{2 k_{j, 2} \cdot k_{j, 1}^{b}} k_{j, 1}^{b, \mu},
$$


so that $\left(k_{j, 1}^{b, \mu}, k_{j, 2}^{b, \mu}\right)$ are massless momenta by construction. It is easy to verify that

$$
k_{j, 1} \cdot k_{j, 2}^{b}=k_{j, 1}^{b} \cdot k_{j, 2}=k_{j, 1}^{b} \cdot k_{j, 2}^{b} .
$$

We streamline notation and define the frequently used quantity

$$
\gamma_{j, 12}=2 k_{j, 1}^{b} \cdot k_{j, 2}^{b}
$$

which upon identification in eq. (3.9) leads to a quadratic equation whose solutions are

$$
\gamma_{j, 12}^{ \pm}=k_{j, 1} \cdot k_{j, 2} \pm\left[\left(k_{j, 1} \cdot k_{j, 2}\right)^{2}-k_{j, 1}^{2} k_{j, 2}^{2}\right]^{1 / 2} .
$$

It is perhaps more useful from a practical point of view to express the massless projections in terms of the corresponding massive momenta. To that end we let $(i, \bar{\imath})$ denote a mutually projecting pair and define

$$
\rho_{j, i}=\frac{k_{j, i}^{2}}{2 k_{j, i} \cdot k_{j, \bar{\imath}}} .
$$

Then it is straightforward to invert eq. (3.9) with the result

$$
k_{j, i}^{b, \mu}=\left(1-\rho_{j, 1} \rho_{j, 2}\right)^{-1}\left(k_{j, i}^{\mu}-\rho_{j, i} k_{j, \bar{\imath}}^{\mu}\right) .
$$

On the other hand, the decomposition of the massive legs in terms of a pair of flattened momenta reads

$$
k_{j, i}^{\mu}=k_{i}^{b, \mu}+\rho_{j, i} k_{\bar{\imath}}^{b, \mu} .
$$

It is convenient to introduce short hand notation $\rho_{12} \equiv \rho_{1,1}, \rho_{21} \equiv \rho_{1,2}$ and $\gamma_{12} \equiv \gamma_{j, 12}$ so that $\rho_{12}=m_{1}^{2} / \gamma_{12}$ and $\rho_{21}=m_{2}^{2} / \gamma_{12}$, and similarly for the other mutually projecting pair. If $m_{1} m_{2}=0$ we have $\gamma_{12}=s_{12}$ and likewise for $\gamma_{34}$. Our final results can therefore be expressed in terms of the nonzero masses among $\left\{m_{1}, m_{2}, m_{3}, m_{4}\right\}, \gamma_{12}$ and $\gamma_{34}$ if respectively $m_{1} m_{2} \neq 0$ and $m_{3} m_{4} \neq 0$, along with two independent Mandelstam invariants, say $s_{12}$ and $s_{14}$.

We adopt a loop momentum parametrization of the form,

$$
\ell_{1}^{\mu}=\frac{1}{2}\left\langle\lambda_{1}^{-}\left|\gamma^{\mu}\right| \tilde{\lambda}_{1}^{\prime-}\right\rangle=\frac{1}{2}\left\langle\lambda_{1}\left|\gamma^{\mu}\right| \tilde{\lambda}_{1}^{\prime}\right], \quad \ell_{2}^{\mu}=\frac{1}{2}\left\langle\lambda_{2}^{-}\left|\gamma^{\mu}\right| \tilde{\lambda}_{2}^{\prime-}\right\rangle=\frac{1}{2}\left\langle\lambda_{2}\left|\gamma^{\mu}\right| \tilde{\lambda}_{2}^{\prime}\right],
$$

so that $\ell_{1}^{2}=\ell_{2}^{2}=0$. The various loop spinors are then constructed from the spinors corresponding to the two mutually projecting pairs with general complex coefficients,

$$
\begin{aligned}
& \left|\lambda_{1}^{+}\right\rangle=\xi_{1}\left|1^{b,+}\right\rangle+\xi_{2} \frac{\left\langle 4^{b} 1^{b}\right\rangle}{\left\langle 4^{b} 2^{b}\right\rangle}\left|2^{b,+}\right\rangle, \quad\left|\tilde{\lambda}_{1}^{\prime-}\right\rangle=\xi_{1}^{\prime}\left|1^{b,-}\right\rangle+\xi_{2}^{\prime} \frac{\left[4^{b} 1^{b}\right]}{\left[4^{b} 2^{b}\right]}\left|2^{b,-}\right\rangle, \\
& \left|\lambda_{2}^{+}\right\rangle=\xi_{3} \frac{\left\langle 1^{b} 4^{b}\right\rangle}{\left\langle 1^{b} 3^{b}\right\rangle}\left|3^{b,+}\right\rangle+\xi_{4}\left|4^{b,+}\right\rangle, \quad\left|\tilde{\lambda}_{2}^{\prime-}\right\rangle=\xi_{3}^{\prime} \frac{\left[1^{b} 4^{b}\right]}{\left[1^{b} 3^{b}\right]}\left|3^{b,-}\right\rangle+\xi_{4}^{\prime}\left|4^{b,-}\right\rangle .
\end{aligned}
$$


In this way, massive external momenta are related to chiral and antichiral spinors corresponding to their massless projections through $2 k_{i}^{\mu}=\left\langle i^{b}\left|\gamma^{\mu}\right| i^{b}\right]$. Expanded explicitly in the basis of four-vectors the two loop momenta read

$$
\begin{aligned}
\ell_{1}^{\mu}\left(\xi_{i}, \xi_{i}^{\prime}\right)= & \xi_{1} \xi_{1}^{\prime} k_{1}^{b, \mu}+\xi_{2} \xi_{2}^{\prime} \frac{k_{1}^{b} \cdot k_{4}^{b}}{k_{2}^{b} \cdot k_{4}^{b} k_{2}^{b, \mu}} \\
& +\frac{\xi_{1} \xi_{2}^{\prime}}{2} \frac{\left[1^{b} 4^{b}\right]}{\left[2^{b} 4^{b}\right]}\left\langle 1^{b,-}\left|\gamma^{\mu}\right| 2^{b,-}\right\rangle+\frac{\xi_{2} \xi_{1}^{\prime}}{2} \frac{\left\langle 1^{b} 4^{b}\right\rangle}{\left\langle 2^{b} 4^{b}\right\rangle}\left\langle 2^{b,-}\left|\gamma^{\mu}\right| 1^{b,-}\right\rangle, \\
\ell_{2}^{\mu}\left(\xi_{i}, \xi_{i}^{\prime}\right)= & \xi_{3} \xi_{3}^{\prime} \frac{k_{1}^{b} \cdot k_{4}^{b}}{k_{1}^{b} \cdot k_{3}^{b}} k_{3}^{b, \mu}+\xi_{4} \xi_{4}^{\prime} k_{4}^{b, \mu} \\
& +\frac{\xi_{3} \xi_{4}^{\prime}}{2} \frac{\left\langle 1^{b} 4^{b}\right\rangle}{\left\langle 1^{b} 3^{b}\right\rangle}\left\langle 3^{b,-}\left|\gamma^{\mu}\right| 4^{b,-}\right\rangle+\frac{\xi_{4} \xi_{3}^{\prime}}{2} \frac{\left[1^{b} 4^{b}\right]}{\left[1^{b} 3^{b}\right]}\left\langle 4^{b,-}\left|\gamma^{\mu}\right| 3^{b,-}\right\rangle,
\end{aligned}
$$

and therefore we are able to eventually fix two of the complex parameters. This freedom amounts sort of a gauge choice. We emphasize that this choice is not necessarily the same for all on-shell branches. In fact, this is not possible in the two-loop crossed box as opposed to the planar case [49].

\subsection{Four-mass hepta-cut equations}

To begin with we will derive the hepta-cut equations for nonzero external masses in all four corners. Besides the on-shell constraints for $\ell_{1}$ and $\ell_{2}$ which are already satisfied automatically, it thus remains to examine the other five massive hepta-cut equations. Three of them are very simple because they only involve one of the loop momenta. Indeed, it takes little effort to realize that

$$
\begin{gathered}
\left(\ell_{1}-k_{1}\right)^{2}=0 \Longrightarrow m_{1}^{2}\left(1-\xi_{1} \xi_{1}^{\prime}\right)-\frac{k_{1}^{b} \cdot k_{4}^{b}}{k_{2}^{b} \cdot k_{4}^{b}} \xi_{2} \xi_{2}^{\prime} \gamma_{12}=0, \\
\left(\ell_{1}-k_{1}-k_{2}\right)^{2}=0 \Longrightarrow s_{12}-m_{1}^{2}-\gamma_{12} \xi_{1} \xi_{1}^{\prime}-\frac{k_{1}^{b} \cdot k_{4}^{b}}{k_{2}^{b} \cdot k_{4}^{b}} m_{2}^{2} \xi_{2} \xi_{2}^{\prime}=0, \\
\left(\ell_{2}-k_{4}\right)^{2}=0 \Longrightarrow m_{4}^{2}\left(1-\xi_{4} \xi_{4}^{\prime}\right)-\frac{k_{1}^{b} \cdot k_{4}^{b}}{k_{1}^{b} \cdot k_{3}^{b}} \xi_{3} \xi_{3}^{\prime} \gamma_{34}=0 .
\end{gathered}
$$

Lorentz products of flattened momenta are needed throughout this calculation. Before we continue let us therefore for completeness derive the necessary expressions. The trick is of course to apply eq. (3.14) and thereby invoke the massive vectors whose contractions are well known. In fact,

$$
\begin{aligned}
k_{1}^{b} \cdot k_{3}^{b}= & \gamma_{12} \gamma_{34}\left(2\left(\gamma_{12}^{2}-m_{1}^{2} m_{2}^{2}\right)\left(\gamma_{34}^{2}-m_{3}^{2} m_{4}^{2}\right)\right)^{-1} \\
\quad \times\left\{m_{1}^{2}\left[\gamma_{34}\left(m_{2}^{2}+m_{3}^{2}-s_{14}\right)+m_{3}^{2}\left(m_{1}^{2}+m_{3}^{2}-s_{12}-s_{14}\right)\right]\right. & \left.\quad+\gamma_{12}\left[m_{3}^{2}\left(m_{1}^{2}+m_{4}^{2}-s_{14}\right)+\gamma_{34}\left(m_{2}^{2}+m_{4}^{2}-s_{12}-s_{14}\right)\right]\right\}, \\
k_{1}^{b} \cdot k_{4}^{b}= & \gamma_{12} \gamma_{34}\left(2\left(\gamma_{12}^{2}-m_{1}^{2} m_{2}^{2}\right)\left(\gamma_{34}^{2}-m_{3}^{2} m_{4}^{2}\right)\right)^{-1} \\
& \times\left\{m_{1}^{2}\left[\gamma_{34}\left(m_{1}^{2}+m_{3}^{2}-s_{12}-s_{14}\right)+m_{4}^{2}\left(m_{2}^{2}+m_{3}^{2}-s_{14}\right)\right]\right. \\
& \left.\quad+\gamma_{12}\left[m_{4}^{2}\left(m_{2}^{2}+m_{4}^{2}-s_{12}-s_{14}\right)+\gamma_{34}\left(m_{1}^{2}+m_{4}^{2}-s_{14}\right)\right]\right\} .
\end{aligned}
$$


We also have

$$
\begin{aligned}
& k_{2}^{b} \cdot k_{3}^{b}= \gamma_{12} \gamma_{34}\left(2\left(\gamma_{12}^{2}-m_{1}^{2} m_{2}^{2}\right)\left(\gamma_{34}^{2}-m_{3}^{2} m_{4}^{2}\right)\right)^{-1} \\
& \quad \times\left\{m_{2}^{2}\left[m_{3}^{2}\left(m_{1}^{2}+m_{4}^{2}-s_{14}\right)+\gamma_{34}\left(m_{2}^{2}+m_{4}^{2}-s_{12}-s_{14}\right)\right]\right. \\
&\left.\quad+\gamma_{12}\left[m_{3}^{2}\left(m_{1}^{2}+m_{3}^{2}-s_{12}-s_{14}\right)+\gamma_{34}\left(m_{2}^{2}+m_{3}^{2}-s_{14}\right)\right]\right\}, \\
& k_{2}^{b} \cdot k_{4}^{b}=\gamma_{12} \gamma_{34}\left(2\left(\gamma_{12}^{2}-m_{1}^{2} m_{2}^{2}\right)\left(\gamma_{34}^{2}-m_{3}^{2} m_{4}^{2}\right)\right)^{-1} \\
& \quad \times\left\{m_{2}^{2}\left[\gamma_{34}\left(m_{1}^{2}+m_{4}^{2}-s_{14}\right)+m_{4}^{2}\left(m_{2}^{2}+m_{4}^{2}-s_{12}-s_{14}\right)\right]\right. \\
&\left.\quad+\gamma_{12}\left[m_{4}^{2}\left(m_{2}^{2}+m_{3}^{2}-s_{14}\right)+\gamma_{34}\left(m_{1}^{2}+m_{3}^{2}-s_{12}-s_{14}\right)\right]\right\} .
\end{aligned}
$$

In addition, we use the same technique to also provide explicit formulas for various contractions of flattened momenta with the external massive legs needed in one of the on-shell equations below,

$$
\begin{aligned}
& k_{1}^{b} \cdot k_{3}=+\frac{\gamma_{12}\left[m_{1}^{2}\left(m_{2}^{2}+m_{3}^{2}-s_{14}\right)+\gamma_{12}\left(m_{2}^{2}+m_{4}^{2}-s_{12}-s_{14}\right)\right]}{2\left(\gamma_{12}^{2}-m_{1}^{2} m_{2}^{2}\right)}, \\
& k_{2}^{b} \cdot k_{3}=-\frac{\gamma_{12}\left[\gamma_{12}\left(m_{2}^{2}+m_{3}^{2}-s_{14}\right)+m_{2}^{2}\left(m_{2}^{2}+m_{4}^{2}-s_{12}-s_{14}\right)\right]}{2\left(\gamma_{12}^{2}-m_{1}^{2} m_{2}^{2}\right)} .
\end{aligned}
$$

Another rather trivial, but useful, identity is $m_{i}^{2}=2 k_{i}^{b} \cdot k_{i}$. Finally, in what proceeds, we will also encounter the quantities

$$
\tau \equiv \frac{\left\langle 1^{b} 4^{b}\right\rangle\left\langle 2^{b} 3^{b}\right\rangle}{\left\langle 2^{b} 4^{b}\right\rangle\left\langle 1^{b} 3^{b}\right\rangle}, \quad \bar{\tau} \equiv \frac{\left[1^{b} 4^{b}\right]\left[2^{b} 3^{b}\right]}{\left[2^{b} 4^{b}\right]\left[1^{b} 3^{b}\right]},
$$

which are complex conjugates of each other for real external momenta as indicated. But actually $\tau=\bar{\tau}$. For completeness, $\tau$ can be expanded and re-expressed in terms of the independent kinematic invariants described above in the following way,

$$
\begin{aligned}
& \tau=\bar{\tau}=- \frac{\gamma_{34}\left(\gamma_{12}+m_{1}^{2}\right)}{\left(\gamma_{34}+m_{3}^{2}\right)\left[\left(\gamma_{12} \gamma_{34}-m_{1}^{2} m_{3}^{2}\right)\left(\gamma_{12} \gamma_{34}-m_{2}^{2} m_{4}^{2}\right)+\gamma_{12} \gamma_{34} s_{12} s_{14}\right]} \\
& \times\left[\left(\gamma_{12}+m_{2}^{2}\right)\left(\gamma_{34}+m_{3}^{2}\right)\left(m_{2}^{2}+m_{3}^{2}-s_{14}\right)+2 m_{2}^{2} m_{3}^{2} s_{12}+\right. \\
& \\
&\left.\quad\left(\gamma_{12}+m_{2}^{2}\right) m_{3}^{2}\left(m_{1}^{2}-m_{2}^{2}-s_{12}\right)+\left(\gamma_{34}+m_{3}^{2}\right) m_{2}^{2}\left(m_{4}^{2}-m_{3}^{2}-s_{12}\right)\right] .
\end{aligned}
$$

Let us now return to the hepta-cut equations for loop momentum $\ell_{1}$. For general masses $m_{1} \neq 0 \neq m_{2}$ we obtain the solution

$$
\xi_{1} \xi_{1}^{\prime}=\frac{\gamma_{12} s_{12}-\left(\gamma_{12}+m_{2}^{2}\right) m_{1}^{2}}{\gamma_{12}^{2}-m_{1}^{2} m_{2}^{2}} \equiv \bar{\xi}_{1}, \quad \xi_{2} \xi_{2}^{\prime}=\frac{m_{1}^{2}\left(m_{1}^{2}+\gamma_{12}-s_{12}\right) k_{2}^{b} \cdot k_{4}^{b}}{\left(\gamma_{12}^{2}-m_{1}^{2} m_{2}^{2}\right) k_{1}^{b} \cdot k_{4}^{b}} \equiv \bar{\xi}_{2} .
$$

In contrast to the planar double box, there is only one additional nontrivial on-shell constraint for loop momentum $\ell_{2}$. Rewriting eq. (3.22) in the slightly more suggestive form,

$$
\xi_{3} \xi_{3}^{\prime}+\mu\left(\xi_{4} \xi_{4}^{\prime}-1\right)=0, \quad \mu \equiv \frac{m_{4}^{2} k_{1}^{b} \cdot k_{3}^{b}}{\gamma_{34} k_{1}^{b} \cdot k_{4}^{b}},
$$

we see that $\xi_{3} \xi_{3}^{\prime}=0$ if $m_{4}=0$ or $\xi_{4} \xi_{4}^{\prime}=1$. 
The foresight in the choice of parametrization of $\ell_{1}$ and $\ell_{2}$ implies that the coupled on-shell equations are also quite compact actually. It happens that one of them factorizes completely in a symmetric manner,

$$
\left(\ell_{1}+\ell_{2}\right)^{2}=0 \Longrightarrow\left(\xi_{1}\left(\xi_{3}+\xi_{4}\right)+\xi_{2}\left(\tau \xi_{3}+\xi_{4}\right)\right) \times\left(\xi_{1}^{\prime}\left(\xi_{3}^{\prime}+\xi_{4}^{\prime}\right)+\xi_{2}^{\prime}\left(\tau \xi_{3}^{\prime}+\xi_{4}^{\prime}\right)\right)=0,
$$

whereas the other can be written

$$
\begin{aligned}
\left(\ell_{1}+\ell_{2}+k_{3}\right)^{2}=0 \Longrightarrow & \\
m_{3}^{2}+2\{ & k_{1}^{b} \cdot k_{3} \xi_{1} \xi_{1}^{\prime}+\left[\tau k_{1}^{b} \cdot k_{3}^{b}+\frac{m_{3}^{2} k_{1}^{b} \cdot k_{4}^{b}}{\gamma_{34}}\right]\left(\xi_{1} \xi_{2}^{\prime}+\xi_{1}^{\prime} \xi_{2}\right) \\
& \left.+\frac{m_{3}^{2} k_{1}^{b} \cdot k_{4}^{b}}{2 k_{1}^{b} \cdot k_{3}^{b}} \xi_{3} \xi_{3}^{\prime}+\frac{k_{1}^{b} \cdot k_{4}^{b} k_{2}^{b} \cdot k_{3}}{k_{2}^{b} \cdot k_{4}^{b}} \xi_{2} \xi_{2}^{\prime}+\frac{\gamma_{34}}{2} \xi_{4} \xi_{4}^{\prime}\right\}=0 .
\end{aligned}
$$

Upon insertion of eqs. (3.31) and (3.32) into eq. (3.34) we immediately find

$$
\begin{aligned}
\xi_{4} \xi_{4}^{\prime}=-\left(\gamma_{34}-\frac{m_{3}^{2} m_{4}^{2}}{\gamma_{34}}\right)^{-1}\left\{m_{3}^{2}\left(1+\frac{m_{4}^{2}}{\gamma_{34}}\right)+2[\right. & k_{1}^{b} \cdot k_{3} \bar{\xi}_{1}+\frac{k_{1}^{b} \cdot k_{4}^{b}}{k_{2}^{b} \cdot k_{4}^{b} k_{2}^{b} \cdot k_{3} \bar{\xi}_{2}} \\
& \left.\left.+\left(\tau k_{1}^{b} \cdot k_{3}^{b}+\frac{m_{3}^{2} k_{1}^{b} \cdot k_{4}^{b}}{\gamma_{34}}\right)\left(\xi_{1} \xi_{2}^{\prime}+\xi_{1}^{\prime} \xi_{2}\right)\right]\right\},
\end{aligned}
$$

or in the slightly more appealing form,

$$
\xi_{4} \xi_{4}^{\prime}=1+\frac{\tau\left(\bar{\xi}_{1}+\bar{\xi}_{2}+\xi_{1} \xi_{2}^{\prime}+\xi_{1}^{\prime} \xi_{2}\right)}{(1-\tau)\left(\bar{\xi}_{1}-\tau \bar{\xi}_{2}\right)}
$$

In the last step we recast the equation by means of the two identities

$$
\begin{aligned}
& \frac{\tau}{(\tau-1)\left(\bar{\xi}_{1}-\tau \bar{\xi}_{2}\right)}=\frac{2 k_{1}^{b} \cdot k_{4}^{b}}{\gamma_{34}^{2}-m_{3}^{2} m_{4}^{2}}\left[m_{3}^{2}+\gamma_{34} \tau \frac{k_{1}^{b} \cdot k_{3}^{b}}{k_{1}^{b} \cdot k_{4}^{b}}\right] \\
& \frac{\bar{\xi}_{1}+\tau^{2} \bar{\xi}_{2}}{(\tau-1)\left(\bar{\xi}_{1}-\tau \bar{\xi}_{2}\right)}=\frac{\gamma_{34}}{\gamma_{34}^{2}-m_{3}^{2} m_{4}^{2}}\left[m_{3}^{2}\left(1+\frac{m_{4}^{2}}{\gamma_{34}}\right)+2\left(k_{1}^{b} \cdot k_{3} \bar{\xi}_{1}+\frac{k_{1}^{b} \cdot k_{4}^{b}}{k_{2}^{b} \cdot k_{4}^{b}} k_{2}^{b} \cdot k_{3} \bar{\xi}_{2}\right)\right]
\end{aligned}
$$

which can be verified through eqs. (3.23)-(3.28) and (3.30) along with expressions for $\bar{\xi}_{1}$ and $\bar{\xi}_{2}$ given in eq. (3.31), although that task is rather tedious.

In order to solve the hepta-cut equations, we decompose the reducible ideal generated by the list of rewritten inverse propagators into an intersection of six prime ideals. Then we compute generating sets that form Gröbner bases over the field of rational functions in each irreducible ideal and obtain the associated zero loci by hand. The six distinct hepta-cut solutions are really three pairs of parity conjugates $\left(\mathcal{S}_{1}, \mathcal{S}_{2}\right),\left(\mathcal{S}_{3}, \mathcal{S}_{4}\right),\left(\mathcal{S}_{5}, \mathcal{S}_{6}\right)$ and each on-shell branch is topologically equivalent to a Riemann sphere, parametrized by a free variable $z \in \mathbb{C}$. 
We choose to make the behavior under parity conjugation manifest and present the solutions in a symmetric manner such that $\mathcal{S}_{2 k-1}$ and $S_{2 k}$ for $k=1,2,3$ are related to each other by simply interchanging $\left(\xi_{1}, \ldots, \xi_{4}\right) \longleftrightarrow\left(\xi_{1}^{\prime}, \ldots, \xi_{4}^{\prime}\right)$. In terms of the parameters $\left(\xi_{1}, \xi_{2}, \xi_{3}, \xi_{4}\right)$ and $\left(\xi_{1}^{\prime}, \xi_{2}^{\prime}, \xi_{3}^{\prime}, \xi_{4}^{\prime}\right)$, the solutions are

$$
\begin{aligned}
\mathcal{S}_{1}:\left(\xi_{1}, \xi_{2}, \xi_{3}, \xi_{4}\right) & =\left(1, z, \frac{\mu \tau(1+z)}{1+\tau z}, 1\right) \\
\left(\xi_{1}^{\prime}, \xi_{2}^{\prime}, \xi_{3}^{\prime}, \xi_{4}^{\prime}\right) & =\left(\bar{\xi}_{1}, \frac{\bar{\xi}_{2}}{z},-\frac{\left(\bar{\xi}_{2}+\bar{\xi}_{1} z\right)(1+\tau z)}{z(1-\tau)\left(\bar{\xi}_{1}-\tau \bar{\xi}_{2}\right)}, \frac{\left(\bar{\xi}_{2}+\bar{\xi}_{1} z\right)(1+\tau z)}{z(1-\tau)\left(\bar{\xi}_{1}-\tau \bar{\xi}_{2}\right)}\right) \\
\mathcal{S}_{2}:\left(\xi_{1}, \xi_{2}, \xi_{3}, \xi_{4}\right) & =\left(\bar{\xi}_{1}, \frac{\bar{\xi}_{2}}{z},-\frac{\left(\bar{\xi}_{2}+\bar{\xi}_{1} z\right)(1+\tau z)}{z(1-\tau)\left(\bar{\xi}_{1}-\tau \bar{\xi}_{2}\right)}, \frac{\left(\bar{\xi}_{2}+\bar{\xi}_{1} z\right)(1+\tau z)}{z(1-\tau)\left(\bar{\xi}_{1}-\tau \bar{\xi}_{2}\right)}\right) \\
\left(\xi_{1}^{\prime}, \xi_{2}^{\prime}, \xi_{3}^{\prime}, \xi_{4}^{\prime}\right) & =\left(1, z, \frac{\mu \tau(1+z)}{1+\tau z}, 1\right) \\
\mathcal{S}_{3}:\left(\xi_{1}, \xi_{2}, \xi_{3}, \xi_{4}\right) & =\left(1,-\frac{\bar{\xi}_{2}}{\bar{\xi}_{1}}, z, 1\right), \quad\left(\xi_{1}^{\prime}, \xi_{2}^{\prime}, \xi_{3}^{\prime}, \xi_{4}^{\prime}\right)=\left(\bar{\xi}_{1},-\bar{\xi}_{1}, 0,1\right) \\
\mathcal{S}_{4}:\left(\xi_{1}, \xi_{2}, \xi_{3}, \xi_{4}\right) & =\left(\bar{\xi}_{1},-\bar{\xi}_{1}, 0,1\right), \quad\left(\xi_{1}^{\prime}, \xi_{2}^{\prime}, \xi_{3}^{\prime}, \xi_{4}^{\prime}\right)=\left(1,-\frac{\bar{\xi}_{2}}{\left.\bar{\xi}_{1}, z, 1\right)}\right. \\
\mathcal{S}_{5}:\left(\xi_{1}, \xi_{2}, \xi_{3}, \xi_{4}\right) & =\left(\bar{\xi}_{1},-\tau \bar{\xi}_{2}, \mu, z\right), \quad\left(\xi_{1}^{\prime}, \xi_{2}^{\prime}, \xi_{3}^{\prime}, \xi_{4}^{\prime}\right)=(1,-1 / \tau, 1,0) \\
\mathcal{S}_{6}:\left(\xi_{1}, \xi_{2}, \xi_{3}, \xi_{4}\right) & =(1,-1 / \tau, 1,0), \quad\left(\xi_{1}^{\prime}, \xi_{2}^{\prime}, \xi_{3}^{\prime}, \xi_{4}^{\prime}\right)=\left(\bar{\xi}_{1},-\tau \bar{\xi}_{2}, \mu, z\right)
\end{aligned}
$$

The branches are written in terms of independent kinematic constants $\bar{\xi}_{1}, \bar{\xi}_{2}, \tau$ and $\mu$ (3.30)-(3.32). For completeness we include the explicit forms of the variables in the four-vector expansions of the two loop momenta for all hepta-cut solutions in appendix A.

\subsection{Massless limits}

We will also analyze the crossed double box with one, two and three massless legs. Integrals for this kinematics are also relevant for higher-multiplicity scattering processes, starting already at five external massless particles.

Let us look more closely at the hepta-cut equations and their solutions. We focus on the two momenta in the crossed end of the diagram and assume that $m_{1} m_{2} \neq 0$. The only dependence on $m_{3}$ is implicitly through other parameters, e.g. $\tau$ and $\mu$. In particular, all on-shell equations and their solutions have the correct limits for $m_{3} \rightarrow 0$. Moreover, it is clear that $\mu \rightarrow 0$ for $m_{4} \rightarrow 0$ so that eq. (3.32) should be replaced by $\xi_{3} \xi_{3}^{\prime}=0$. It can be shown that the number of branches remains six and the explicit solutions follow from the four-mass case once we let $\mu \rightarrow 0$. The configurations corresponding to this class of kinematics are illustrated in figure 2 .

The situation is slightly more complicated when momenta $k_{1}$ and $k_{2}$ in the planar end become massless. Here, the massless limits are not smooth and hence they should be treated carefully. We see that $\bar{\xi}_{1} \rightarrow 1$ as $m_{2} \rightarrow 0$ and $m_{1}$ arbitrary. Also, $\bar{\xi}_{1} \rightarrow 1+m_{2}^{2} / \gamma_{12}$ when $m_{1} \rightarrow 0$. Therefore the equation $\xi_{1} \xi_{1}^{\prime}-\bar{\xi}_{1}=0$ will not give rise to branchings. But if at least one mass among $\left\{m_{1}, m_{2}\right\}$ is zero, $\bar{\xi}_{2}=0$ and we instead get the equation $\xi_{2} \xi_{2}^{\prime}=0$ and eq. (3.31) must be replaced by a pair of solutions, i.e. $\xi_{2}^{\prime}=0$ and $\xi_{2}$ free or vice versa. 

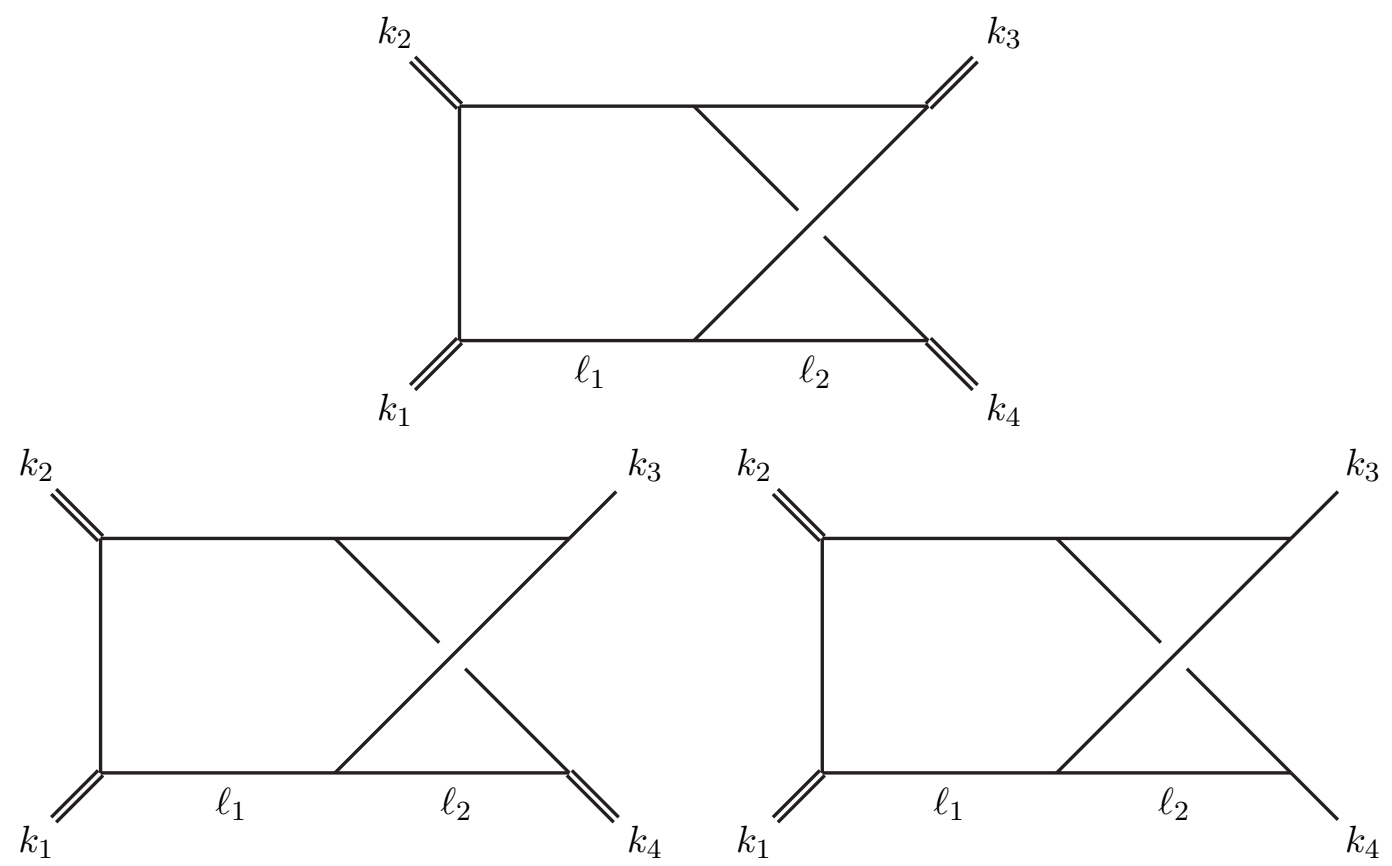

Figure 2. The first class of two-loop crossed-box integrals includes the four-mass case and related massless limits, i.e. three-mass and short-side two-mass with massless legs in the nonplanar end of the diagram. Massless and massive external legs are denoted by single and doubled lines respectively.

We solved the hepta-cut equations for this class of kinematics and found eight distinct solutions that can be parametrized as follows,

$$
\begin{aligned}
& \widetilde{\mathcal{S}}_{1}:\left(\xi_{1}, \xi_{2}, \xi_{3}, \xi_{4}\right)=\left(1, z, \frac{\mu \tau(1+z)}{1+\tau z}, 1\right), \quad\left(\xi_{1}^{\prime}, \xi_{2}^{\prime}, \xi_{3}^{\prime}, \xi_{4}^{\prime}\right)=\left(\bar{\xi}_{1}, 0,-\frac{1+\tau z}{1-\tau}, \frac{1+\tau z}{1-\tau}\right), \\
& \widetilde{\mathcal{S}}_{2}:\left(\xi_{1}, \xi_{2}, \xi_{3}, \xi_{4}\right)=\left(\bar{\xi}_{1}, 0,-\frac{1+\tau z}{1-\tau}, \frac{1+\tau z}{1-\tau}\right), \quad\left(\xi_{1}^{\prime}, \xi_{2}^{\prime}, \xi_{3}^{\prime}, \xi_{4}^{\prime}\right)=\left(1, z, \frac{\mu \tau(1+z)}{1+\tau z}, 1\right), \\
& \widetilde{\mathcal{S}}_{3}:\left(\xi_{1}, \xi_{2}, \xi_{3}, \xi_{4}\right)=\left(1, z,-\frac{1+z}{1+\tau z}, 1\right), \quad\left(\xi_{1}^{\prime}, \xi_{2}^{\prime}, \xi_{3}^{\prime}, \xi_{4}^{\prime}\right)=\left(\bar{\xi}_{1}, 0, \frac{\mu \tau(1+\tau z)}{\tau-1}, \frac{1+\tau z}{1-\tau}\right), \\
& \widetilde{\mathcal{S}}_{4}:\left(\xi_{1}, \xi_{2}, \xi_{3}, \xi_{4}\right)=\left(\bar{\xi}_{1}, 0, \frac{\mu \tau(1+\tau z)}{\tau-1}, \frac{1+\tau z}{1-\tau}\right), \quad\left(\xi_{1}^{\prime}, \xi_{2}^{\prime}, \xi_{3}^{\prime}, \xi_{4}^{\prime}\right)=\left(1, z,-\frac{1+z}{1+\tau z}, 1\right), \\
& \widetilde{\mathcal{S}}_{5}:\left(\xi_{1}, \xi_{2}, \xi_{3}, \xi_{4}\right)=(1,0, z, 1), \quad\left(\xi_{1}^{\prime}, \xi_{2}^{\prime}, \xi_{3}^{\prime}, \xi_{4}^{\prime}\right)=\left(\bar{\xi}_{1},-\bar{\xi}_{1}, 0,1\right), \\
& \widetilde{\mathcal{S}}_{6}:\left(\xi_{1}, \xi_{2}, \xi_{3}, \xi_{4}\right)=\left(\bar{\xi}_{1},-\bar{\xi}_{1}, 0,1\right), \quad\left(\xi_{1}^{\prime}, \xi_{2}^{\prime}, \xi_{3}^{\prime}, \xi_{4}^{\prime}\right)=(1,0, z, 1), \\
& \widetilde{\mathcal{S}}_{7}:\left(\xi_{1}, \xi_{2}, \xi_{3}, \xi_{4}\right)=\left(\bar{\xi}_{1}, 0, \mu, z\right), \quad\left(\xi_{1}^{\prime}, \xi_{2}^{\prime}, \xi_{3}^{\prime}, \xi_{4}^{\prime}\right)=(1,-1 / \tau, 1,0), \\
& \widetilde{\mathcal{S}}_{8}:\left(\xi_{1}, \xi_{2}, \xi_{3}, \xi_{4}\right)=(1,-1 / \tau, 1,0), \quad\left(\xi_{1}^{\prime}, \xi_{2}^{\prime}, \xi_{3}^{\prime}, \xi_{4}^{\prime}\right)=\left(\bar{\xi}_{1}, 0, \mu, z\right) .
\end{aligned}
$$

Notice that six of the tilded solutions are inherited from the four-mass case in the limit $\bar{\xi}_{2} \rightarrow 0$. The new branches are denoted $\widetilde{\mathcal{S}}_{3}$ and $\widetilde{\mathcal{S}}_{4}$. We invite the reader to refer 

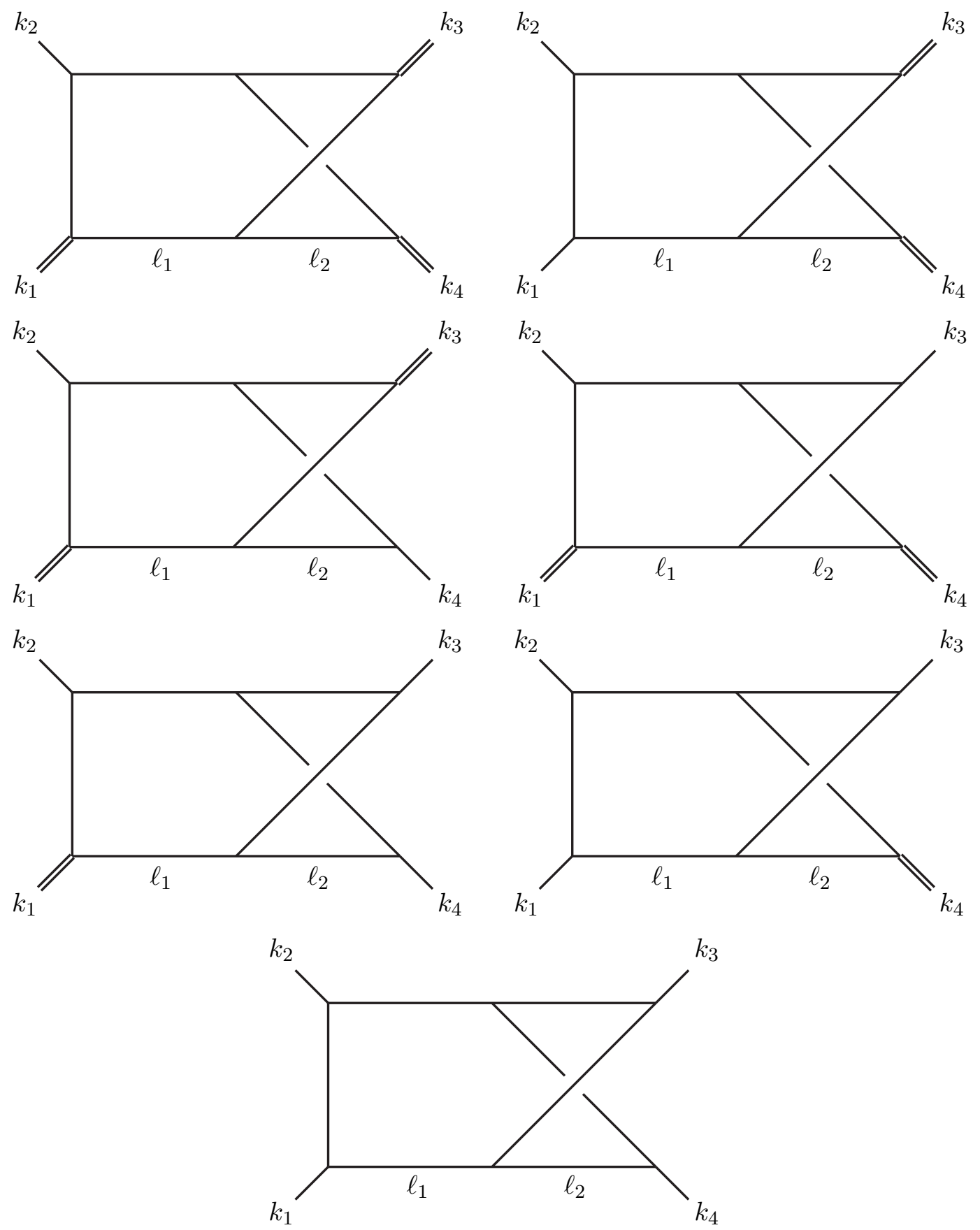

Figure 3. The three-mass and the two-mass short-side integrals with massless legs in the planar end of the diagram together with diagonal and long-side two-mass integrals, one-mass integrals and finally the zero-mass integral correspond to degenerate massless limits.

to appendix A for the full expressions of the loop momenta on each branch. The various kinematic configurations are depicted in figures 2 and 3. 

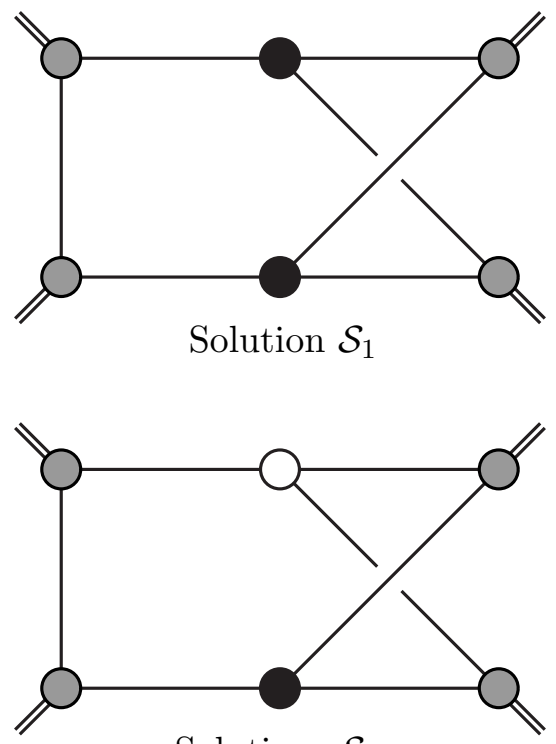

Solutions $\mathcal{S}_{3,5}$
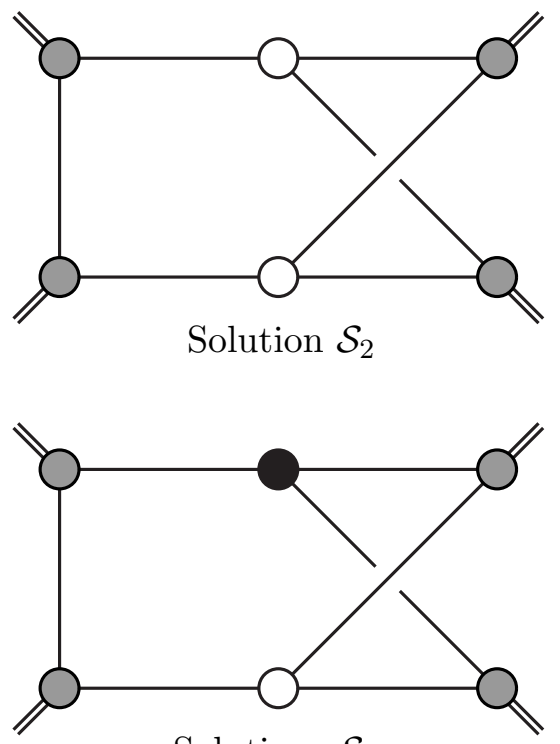

Solutions $\mathcal{S}_{4,6}$

Figure 4. The four possible vertex configurations of the four-mass two-loop crossed box. Black, white and gray blobs denote chiral, antichiral and nonchiral vertices respectively. The oppositechirality diagrams are not in one-to-one correspondence with the hepta-cut solutions.

\subsection{Classification of kinematic solutions}

The degeneracy of maximal-cut solutions is customarily understood diagrammatically from the possible distributions of chiralities at the on-shell vertices. Momentum conservation in a three-point vertex forces either square or angle spinor products to align. Phrased slightly differently, the positive or negative chirality spinors of the momenta are collinear, $\lambda_{a} \propto \lambda_{b} \propto \lambda_{c}$ or $\tilde{\lambda}_{a} \propto \tilde{\lambda}_{b} \propto \tilde{\lambda}_{c}$. We choose to depict such vertices as $\circ$ and $\bullet$ and refer to them as antichiral ( $\overline{\mathrm{MHV}}$ ) and chiral (MHV) respectively. Vertices involving more than three particles or massive momenta do not have a well-defined chirality.

In recent studies, a one-to-one correspondence between diagrams and kinematic solutions was found for the planar double box with up to four massive legs [47-49, 53], the two-loop crossed box [50] and the planar three-loop triple box [51].

The situation is different for the two-loop crossed box. Consider the four-mass case which we know gives rise to six classes of hepta-cut solutions. The maximal cut leaves two massless on-shell three-vertices in a 2-mass-easy sub-box and hence we would erroneously predict only four solutions based on the diagrams in figure 4 . The cause of this mismatch is that solutions $\mathcal{S}_{3}$ and $\mathcal{S}_{5}$ actually correspond to the same opposite-chirality diagram and similarly for $\mathcal{S}_{4}$ and $\mathcal{S}_{6}$.

\section{Residues of the loop integrand and topological structure}

The internal momenta have eight degrees of freedom in strictly four dimensions and therefore we are left with one free parameter, after imposing the hepta-cut constraints. This 
implies that after promoting the original real slice contours of integration to seven-tori encircling the simultaneous zeros of the denominators in the crossed box integral, the integrand is localized onto the Riemann sphere associated with one of the hepta-cut equations found in the previous section.

The maximal cut involves an inverse Jacobian that has multiple poles in the remaining variable $z$. These poles are known as composite leading singularities. For integrals with tensor numerators, additional poles in the integrand must be taken into account. Once the pole structure is properly understood, we can define the hepta-cut integral by a weighted residue expansion over a minimal set of all poles. We return to this part of the computation shortly.

In order to actually perform the hepta-cut contour integrals, it is necessary to break the automatic satisfaction of the on-shell constraints $\ell_{1}^{2}=\ell_{2}^{2}=0$ which instead should be imposed by localization. To that end it is advantageous to introduce new parameters $\zeta_{1}, \zeta_{2} \in \mathbb{C}$ and two null-vectors $\eta_{1}, \eta_{2}$ with the obvious properties,

$$
\eta_{1}^{\mu}\left|\lambda_{1}^{+}\right\rangle \neq 0 \neq \eta_{1}^{\mu}\left|\tilde{\lambda}_{1}^{\prime-}\right\rangle, \quad \eta_{2}^{\mu}\left|\lambda_{2}^{+}\right\rangle \neq 0 \neq \eta_{1}^{\mu}\left|\tilde{\lambda}_{2}^{\prime-}\right\rangle
$$

and then shift the loop momentum parametrization such that

$$
\ell_{1}^{\mu}=\frac{1}{2}\left\langle\lambda_{1}\left|\gamma^{\mu}\right| \tilde{\lambda}_{1}^{\prime}\right]+\zeta_{1} \eta_{1}^{\mu}, \quad \ell_{2}^{\mu}=\frac{1}{2}\left\langle\lambda_{2}\left|\gamma^{\mu}\right| \tilde{\lambda}_{2}^{\prime}\right]+\zeta_{2} \eta_{2}^{\mu} .
$$

In general, this choice is tied to the specific on-shell solution in question. An appropriate choice in any case is to take the masslessly projected legs because it simplifies the calculation. For instance, let $\eta_{1}=k_{2}^{b}$ and $\eta_{2}=k_{3}^{b}$. Then for all four-mass solutions $\mathcal{S}_{1}, \ldots, \mathcal{S}_{4}$ it is clear that

$$
\ell_{1}^{2}=\gamma_{12} \xi_{1} \xi_{1}^{\prime} \zeta_{1}=0, \quad \ell_{2}^{2}=\gamma_{34} \xi_{4} \xi_{4}^{\prime} \zeta_{2}=0
$$

and therefore we obtain the desired solution $\zeta_{1}=\zeta_{2}=0$ since $\xi_{1} \xi_{1}^{\prime} \neq 0 \neq \xi_{4} \xi_{4}^{\prime}$ for general momenta. The same arguments show that a valid choice for solutions $\mathcal{S}_{5}$ and $\mathcal{S}_{6}$ is $\eta_{1}=k_{2}^{b}$ and $\eta_{2}=k_{4}^{b}$ because in that situation $\xi_{1} \xi_{1}^{\prime} \neq 0 \neq \xi_{3} \xi_{3}^{\prime}$.

We also need to include the Jacobian $J^{L} \times J^{R}$ due to coordinate transformation from loop four-momenta to parameter space. This can easily be done by Wick rotation to Euclidean spacetime where the volume of the 4-parallelotope spanned by a set of four vectors $q_{i}^{\mu}$ can be computed from the corresponding Gram determinant up to an overall sign, which we eventually determine numerically along with potential factors of $i$ introduced by the analytic continuation,

$$
\operatorname{det}_{\mu, i} q_{i}^{\mu}= \pm\left(\operatorname{det}_{i, j} q_{i} \cdot q_{j}\right)^{1 / 2}
$$

The set of variables parametrizing the two loop momenta $\ell_{1}$ and $\ell_{2}$ after removal of redundant degrees of freedom also depend on the branch in question. In $\mathcal{S}_{1}$ and $\mathcal{S}_{3}$ we keep the variables $\alpha=\left(\zeta_{1}, \xi_{1}^{\prime}, \xi_{2}, \xi_{2}^{\prime}\right)$ and $\beta=\left(\zeta_{2}, \xi_{3}, \xi_{3}^{\prime}, \xi_{4}\right)$ and thus fix $\xi_{1}=\xi_{4}^{\prime}=1$. The corresponding Jacobians to appear in the numerator are then

$$
J_{\mathcal{S}_{1,3}}^{L}=\operatorname{det}_{\mu, i} \frac{\partial \ell_{1}^{\mu}}{\partial \alpha_{i}}=i \frac{\gamma_{12}^{2} k_{1}^{b} \cdot k_{4}^{b} \xi_{1}^{\prime}}{4 k_{2}^{b} \cdot k_{4}^{b}}, \quad J_{\mathcal{S}_{1,3}^{R}}^{R}=\operatorname{det}_{\mu, i} \frac{\partial \ell_{2}^{\mu}}{\partial \beta_{i}}=i \frac{\gamma_{34}^{2} k_{1}^{b} \cdot k_{4}^{b} \xi_{4}^{\prime}}{4 k_{1}^{b} \cdot k_{3}^{b}} .
$$


Notice that these forms are not constant because the parametrization is not linear in the parameters as opposed to previous work [50] in the purely massless case. Similarly for $\mathcal{S}_{2}$ and $\mathcal{S}_{4}$ where $\alpha=\left(\zeta_{1}, \xi_{1}, \xi_{2}, \xi_{2}^{\prime}\right)$ and $\beta=\left(\zeta_{2}, \xi_{3}, \xi_{3}^{\prime}, \xi_{4}^{\prime}\right)$ so that

$$
J_{\mathcal{S}_{2,4}}^{L}=\operatorname{det}_{\mu, i} \frac{\partial \ell_{1}^{\mu}}{\partial \alpha_{i}}=i \frac{\gamma_{12}^{2} k_{1}^{b} \cdot k_{4}^{b} \xi_{1}}{4 k_{2}^{b} \cdot k_{4}^{b}}, \quad J_{\mathcal{S}_{2,4}^{R}}^{R}=\operatorname{det}_{\mu, i} \frac{\partial \ell_{2}^{\mu}}{\partial \beta_{i}}=i \frac{\gamma_{34}^{2} k_{1}^{b} \cdot k_{4}^{b} \xi_{4}}{4 k_{1}^{b} \cdot k_{3}^{b}}
$$

We finally compute the Jacobians appropriate to solutions $\mathcal{S}_{5}$ and $\mathcal{S}_{6}$,

$$
\begin{aligned}
& J_{\mathcal{S}_{5}}^{L}=\operatorname{det}_{\mu, i} \frac{\partial \ell_{1}^{\mu}}{\partial \alpha_{i}}=i \frac{\gamma_{12}^{2} k_{1}^{b} \cdot k_{4}^{b} \xi_{1}}{4 k_{2}^{b} \cdot k_{4}^{b}}, \quad J_{\mathcal{S}_{5}}^{R}=\operatorname{det} \frac{\partial \ell_{2}^{\mu}}{\partial \beta_{i}}=i \gamma_{34}^{2}\left(\frac{k_{1}^{b} \cdot k_{4}^{b}}{k_{1}^{b} \cdot k_{3}^{b}}\right)^{2} \xi_{3}, \\
& J_{\mathcal{S}_{6}}^{L}=\operatorname{det}_{\mu, i} \frac{\partial \ell_{1}^{\mu}}{\partial \alpha_{i}}=i \frac{\gamma_{12}^{2} k_{1}^{b} \cdot k_{4}^{b} \xi_{1}^{\prime}}{4 k_{2}^{b} \cdot k_{4}^{b}}, \quad J_{\mathcal{S}_{6}}^{R}=\operatorname{det}_{\mu, i} \frac{\partial \ell_{2}^{\mu}}{\partial \beta_{i}}=i \gamma_{34}^{2}\left(\frac{k_{1}^{b} \cdot k_{4}^{b}}{k_{1}^{b} \cdot k_{3}^{b}}\right)^{2} \xi_{3}^{\prime} .
\end{aligned}
$$

\subsection{Hextuply pinched genus-3 curve}

The preceding discussions now lead us to the definition of localization of the two-loop crossed-box integral onto the Riemann sphere associated with the $i$ th branch. Changing integration variables from loop momenta $\left(\ell_{1}^{\mu}, \ell_{2}^{\mu}\right)$ to parameters $\left(\xi_{i}, \xi_{i}^{\prime}\right)$, replacing real slice integration contours by a multidimensional tori encircling the joint solution of the heptacut constraints and subsequently performing seven contour integrals using eq. (2.9) give rise to a total Jacobian $J_{i}$,

$$
X_{2,1,1}^{* *}[1]_{\mathcal{S}_{i}} \equiv \oint_{\Gamma_{i}} d z J_{i}(z)
$$

The generic form of this Jacobian is a product of $n_{i}$ simple-pole factors associated with the pinching points or intersections with neighboring Riemann spheres,

$$
J_{i} \equiv \frac{h(z)}{\prod_{k=1}^{n_{i}}\left(z-z_{i ; k}\right)},
$$

where $h(z)$ is a regular function of $z$. For the two-loop crossed box integrals with up to four massive legs and no doubled propagators, the Jacobian will at most define a quartic polynomial because $n_{i} \leq 4$ for all $i$ as we shall see below.

It is straightforward to obtain the Jacobians explicitly after the inverse propagators have been expanded in parameter space. For brevity we merely state the results here. We refer the reader to e.g. refs. [46, 50, 51] for related examples. In advance of calculations below, we identify a frequently occurring kinematic constant along the lines of ref. [49],

$$
\gamma_{\star} \equiv \frac{\gamma_{12} \gamma_{34}}{32\left(\gamma_{12}^{2}-m_{1}^{2} m_{2}^{2}\right)\left(\gamma_{34}^{2}-m_{3}^{2} m_{4}^{2}\right) k_{1}^{b} \cdot k_{4}^{b}}
$$


The multivariate residues evaluated at the simultaneous zeros of the denominators are,

$$
\begin{aligned}
& X_{2,1,1}^{* *}[1]_{\mathcal{S}_{1,2}}=+\frac{\gamma_{\star}(1-\tau)\left(\bar{\xi}_{1}-\tau \bar{\xi}_{2}\right)}{\tau \bar{\xi}_{1}^{2}(1+\mu \tau)} \oint d z \frac{z}{(z+1)\left(z+\frac{1}{\tau}\right)\left(z+\frac{\bar{\xi}_{2}}{\bar{\xi}_{1}}\right)\left(z+\frac{\tau \bar{\xi}_{2}}{\bar{\xi}_{1}}\right)}, \\
& X_{2,1,1}^{* *}[1]_{\mathcal{S}_{3,4}}=-\frac{\gamma_{\star}}{\bar{\xi}_{1}-\tau \bar{\xi}_{2}} \oint \frac{d z}{\left(z+\frac{\bar{\xi}_{1}-\bar{\xi}_{2}}{\bar{\xi}_{1}-\tau \bar{\xi}_{2}}\right)\left(z-\frac{\mu \tau\left(\bar{\xi}_{1}-\bar{\xi}_{2}\right)}{\bar{\xi}_{1}-\tau \bar{\xi}_{2}}\right)}, \\
& X_{2,1,1}^{* *}[1]_{\mathcal{S}_{5,6}}=+\frac{\gamma_{\star}}{\bar{\xi}_{1}-\tau \bar{\xi}_{2}} \oint \frac{d z}{\left(z-\frac{\bar{\xi}_{1}-\tau^{2} \bar{\xi}_{2}}{\tau\left(\bar{\xi}_{1}-\tau \bar{\xi}_{2}\right)}\right)\left(z+\frac{\mu\left(\bar{\xi}_{1}-\tau^{2} \bar{\xi}_{2}\right)}{\left.\bar{\xi}_{1}-\tau \bar{\xi}_{2}\right)}\right)}
\end{aligned}
$$

where the pole locations in the Jacobians are directly exposed,

$$
\begin{aligned}
\left\{z_{1 ; 1}, \ldots, z_{1 ; 4}\right\} & =\left\{-1,-\frac{1}{\tau},-\frac{\bar{\xi}_{2}}{\bar{\xi}_{1}},-\frac{\tau \bar{\xi}_{2}}{\bar{\xi}_{1}}\right\}=\left\{z_{2 ; 1}, \ldots, z_{2 ; 4}\right\} \\
\left\{z_{3 ; 1}, z_{3 ; 2}\right\} & =\left\{-\frac{\bar{\xi}_{1}-\bar{\xi}_{2}}{\bar{\xi}_{1}-\tau \bar{\xi}_{2}}, \frac{\mu \tau\left(\bar{\xi}_{1}-\bar{\xi}_{2}\right)}{\bar{\xi}_{1}-\tau \bar{\xi}_{2}}\right\}=\left\{z_{4 ; 1}, z_{4 ; 2}\right\}, \\
\left\{z_{5 ; 1}, z_{5 ; 2}\right\} & =\left\{\frac{\bar{\xi}_{1}-\tau^{2} \bar{\xi}_{2}}{\tau\left(\bar{\xi}_{1}-\tau \bar{\xi}_{2}\right)},-\frac{\mu\left(\bar{\xi}_{1}-\tau^{2} \bar{\xi}_{2}\right)}{\bar{\xi}_{1}-\tau \bar{\xi}_{2}}\right\}=\left\{z_{6 ; 1}, z_{6 ; 2}\right\} .
\end{aligned}
$$

Solutions $\mathcal{S}_{1}$ and $\mathcal{S}_{2}$ give rise to additional singularities where either of the loop momenta become infinite for a finite value of the post hepta-cut degree of freedom $z$. Finally, in each solution there is a pole at $z=\infty$ which may be encircled. The union of the singular point loci for all six branches therefore contains 24 points.

This leads to the definition of the octa-cut of a general tensor integral with numerator insertion $\Phi\left(\ell_{1}(z), \ell_{2}(z)\right)$,

$$
X_{2,1,1}^{* *}\left[\left.\Phi\left(\ell_{1}(z), \ell_{2}(z)\right]\right|_{8-\mathrm{cut}} \equiv \sum_{i=1}^{6} \oint_{\Gamma_{i}} d z J_{i}(z) \Phi\left(\ell_{1}(z), \ell_{2}(z)\right),\right.
$$

Here, $\Gamma_{i}$ is a weighted linear combination of small circles around the poles in the remaining variable $z$ chosen so that the integrals extract the residues of the loop integrand. Denoting the weight of the residue evaluated at $z=\xi$ for the $i$ th branch by $\omega(i, \xi)$, we have

$$
\left.X_{2,1,1}^{* *}\left[\Phi\left(\ell_{1}(z), \ell_{2}(z)\right)\right]\right|_{8-\text { cut }}=\left.\sum_{i=1}^{|\mathcal{S}|} \sum_{\xi \in \text { poles }} \omega(i, \xi) \underset{z=\xi}{\operatorname{Res}} J_{i}(z) \Phi(z)\right|_{\mathcal{S}_{i}} .
$$

Not all of these residues are independent though, as can be explained from the global structure of the unitarity cut $[47,64]$. Indeed, consider an arbitrary integrand test function of the two loop momenta, $\Phi\left(\ell_{1}(z), \ell_{2}(z)\right)$, and assume regularity on the Jacobian poles. It is then very easy to prove the residue relation,

$$
\left.\underset{\mathcal{S}_{i} \cap S_{j}}{\operatorname{Res}} J(z) \Phi\left(\ell_{1}(z), \ell_{2}(z)\right)\right|_{\mathcal{S}_{i}}=-\left.\operatorname{Res}_{\mathcal{S}_{i} \cap S_{j}} J(z) \Phi\left(\ell_{1}(z), \ell_{2}(z)\right)\right|_{\mathcal{S}_{j}},
$$

where the left and right hand sides of the equation are understood to be evaluated in local coordinates on solutions $\mathcal{S}_{i}$ and $\mathcal{S}_{j}$ respectively. Other choices are equally valid, e.g symmetric in $i$ and $j$. For the purpose of completeness, let us state all such identities: 


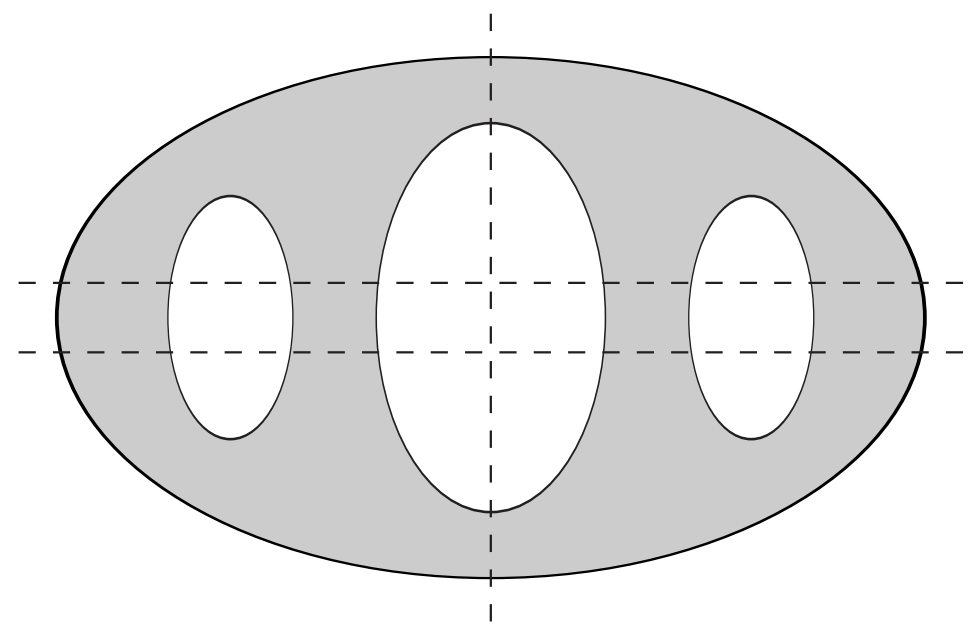

Figure 5. Topological depiction of the genus-3 algebraic curve defined by the hepta-cut of the prime configuration of the two-loop crossed box primitive amplitude. The one-dimensional complex curve should be understood as the filled two-dimensional real surface. Degeneracies appropriate to specific kinematics arise upon contraction of tubes along straight horizontal and vertical lines in the paper plane through the handles of the surface.

$$
\begin{gathered}
\left.\underset{z=z_{1,1}}{\operatorname{Res}} J_{1}(z) \Phi(z)\right|_{\mathcal{S}_{1}}=-\left.\operatorname{Res}_{z=z_{4,1}} J_{4}(z) \Phi(z)\right|_{\mathcal{S}_{4}},\left.\quad \operatorname{Res}_{z=z_{2,1}} J_{2}(z) \Phi(z)\right|_{\mathcal{S}_{2}}=-\left.\operatorname{Res}_{z=z_{3,1}} J_{3}(z) \Phi(z)\right|_{\mathcal{S}_{3}}, \\
\left.\underset{z=z_{1,2}}{\operatorname{Res}} J_{1}(z) \Phi(z)\right|_{\mathcal{S}_{1}}=-\left.\operatorname{Res}_{z=z_{6,2}} J_{6}(z) \Phi(z)\right|_{\mathcal{S}_{6}},\left.\quad \underset{z=z_{2,2}}{\operatorname{Res}} J_{2}(z) \Phi(z)\right|_{\mathcal{S}_{2}}=-\left.\operatorname{Res}_{z=z_{5,2}} J_{5}(z) \Phi(z)\right|_{\mathcal{S}_{5}}, \\
\left.\operatorname{Res}_{z=z_{1,3}} J_{1}(z) \Phi(z)\right|_{\mathcal{S}_{1}}=-\left.\operatorname{Res}_{z=z_{3,2}} J_{3}(z) \Phi(z)\right|_{\mathcal{S}_{3}},\left.\quad \underset{z=z_{2,3}}{\operatorname{Res}} J_{2}(z) \Phi(z)\right|_{\mathcal{S}_{2}}=-\left.\operatorname{Res}_{z=z_{4,2}} J_{4}(z) \Phi(z)\right|_{\mathcal{S}_{4}}, \\
\left.\underset{z=z_{1,4}}{\operatorname{Res}} J_{1}(z) \Phi(z)\right|_{\mathcal{S}_{1}}=-\left.\operatorname{Res}_{z=z_{5,1}} J_{5}(z) \Phi(z)\right|_{\mathcal{S}_{5}},\left.\quad \operatorname{Res}_{z=z_{2,4}} J_{2}(z) \Phi(z)\right|_{\mathcal{S}_{2}}=-\left.\operatorname{Res}_{z=z_{6,1}} J_{6}(z) \Phi(z)\right|_{\mathcal{S}_{6}} .
\end{gathered}
$$

This pattern of intersections confirms the global topological structure of the hepta-cut in figure 6 . This picture follows by pinching the tubes of the genus- 3 surface six times along a horizontal and a vertical line passing through the center of the object, see figure 5 . The number of independent residues is reduced to 16 . The residues at infinity and in numerator insertions are not shared. However, within each Riemann sphere the one-dimensional global residue theorem ensures that the sum of all residues vanish.

The upshot is that we only need to encircle 10 global poles to produce a complete basis of homology for $\mathcal{S}_{1} \cup \cdots \cup \mathcal{S}_{6}$. In particular, the contributions from branches $\mathcal{S}_{3}, \ldots, \mathcal{S}_{6}$ are redundant, because each of them only has a single pole $($ at $z=\infty$ ) besides those located at intersections with $\mathcal{S}_{1}$ and $\mathcal{S}_{2}$. To minimize an overcomplete basis we may simply set extraneous residue weights to zero. It is natural to choose an ordered set of winding numbers, call it $\boldsymbol{\Omega}$, so that we encircle all Jacobian poles along with poles in numerator insertions where both loop momenta become infinite simultaneously. Following the notation 

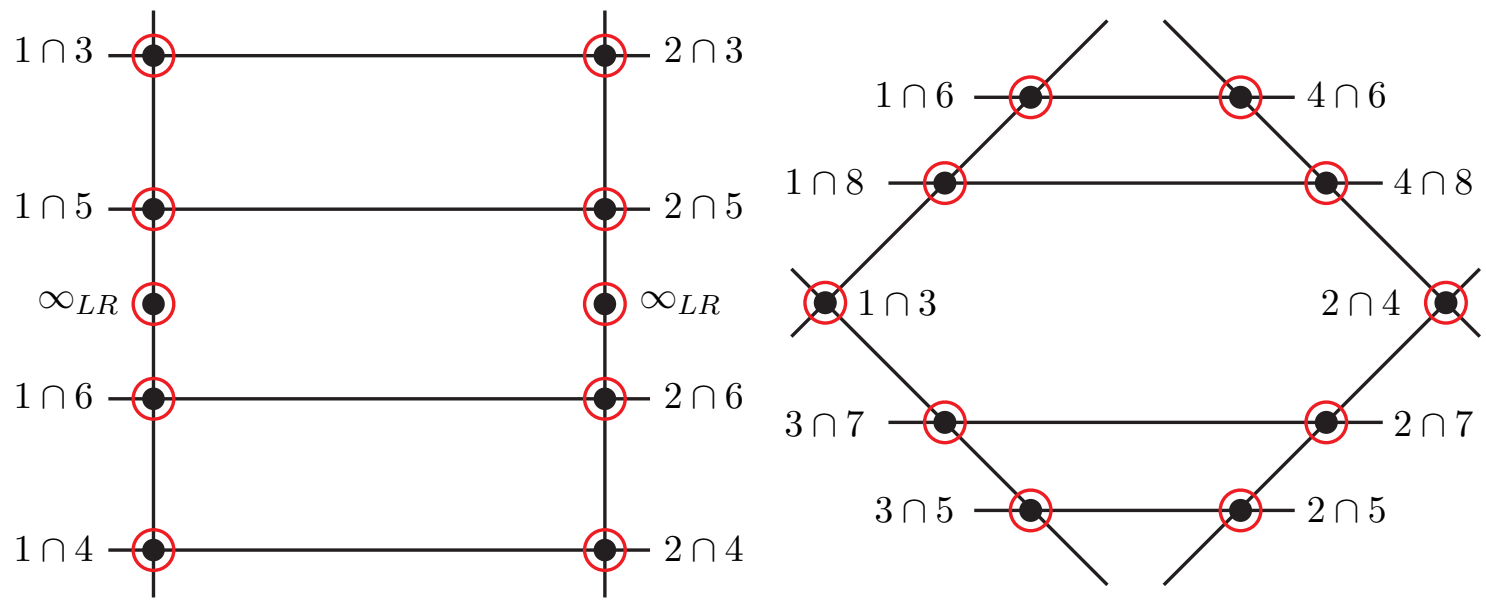

Figure 6. Global structure of the hepta-cut of the two-loop crossed box; the figures show intersections of the irreducible components of the algebraic curve defined by the zero locus of the polynomial ideal generated by the inverse propagators in the case of four external massive legs (left) and at least one massless particle (right). All degenerate configurations considered in this paper fall within these two topological pictures. The straight lines are drawn for simplicity and should be interpreted as Riemann spheres.

of ref. [47], the weights of the ten global poles can be written

$$
\boldsymbol{\Omega}=\left(\omega_{1 \cap 4}, \omega_{1 \cap 6}, \omega_{1 \cap 3}, \omega_{1 \cap 5}, \omega_{1, \infty_{L R}}, \omega_{2 \cap 3}, \omega_{2 \cap 5}, \omega_{2 \cap 4}, \omega_{2 \cap 6}, \omega_{1, \infty_{L R}}\right) \equiv\left(\omega_{1}, \ldots, \omega_{10}\right)
$$

By convention, a residue with weight $\omega_{i \cap j}$ is evaluated on the $i$ th branch. Later it may be convenient to instead encircle infinity poles, since the scalar integrals yield simpler residues there.

\subsection{Octuply pinched genus-3 curve}

Let us now relax the condition $m_{1} m_{2} \neq 0$ and analyze the analytic structure of the loop integrand under those circumstances. The eight hepta-cut solutions for this class of kinematics were determined in section 3.3. The topological picture is that of an octuply pinched genus-3 surface, where tubes have been contracted along one vertical and two horizontal lines through the center as shown in figure 5 .

We can reproduce this situation locally from coincidence of residues. Localizing the scalar master integral onto each of the eight Riemann spheres parametrized by the heptacut solutions $\widetilde{\mathcal{S}}_{1}$ through $\widetilde{\mathcal{S}}_{8}$ yields four pairs of one-dimensional contour integrals,

$$
\begin{aligned}
& X_{2,1,1}^{* *}[1]_{\widetilde{\mathcal{S}}_{1,2}}=+\frac{\gamma_{\star}(1-\tau)}{\tau \bar{\xi}_{1}(1+\mu \tau)} \oint \frac{d z}{z(z+1)(z+1 / \tau)}, \\
& X_{2,1,1}^{* *}[1]_{\widetilde{\mathcal{S}}_{3,4}}=-\frac{\gamma_{\star}(1-\tau)}{\tau \bar{\xi}_{1}(1+\mu \tau)} \oint \frac{d z}{z(z+1)(z+1 / \tau)},
\end{aligned}
$$




$$
\begin{aligned}
& X_{2,1,1}^{* *}[1]_{\widetilde{\mathcal{S}}_{5,6}}=-\frac{\gamma_{\star}}{\bar{\xi}_{1}} \oint \frac{d z}{(z+1)(z-\mu \tau)}, \\
& X_{2,1,1}^{* *}[1]_{\widetilde{\mathcal{S}}_{7,8}}=+\frac{\gamma_{\star}}{\bar{\xi}_{1}} \oint \frac{d z}{(z+\mu)(z-1 / \tau)} .
\end{aligned}
$$

The residues at the poles in the displayed loop integrands again satisfy a rich set of linear relations across the irreducible components of the genus-3 curve and thus reflect the global structure of the unitarity cut. In fact,

$$
\begin{aligned}
& \left.\operatorname{Res}_{z=0} \widetilde{J}_{1}(z) \Phi(z)\right|_{\widetilde{\mathcal{S}}_{1}}=-\left.\operatorname{Res}_{z=0} \widetilde{J}_{3}(z) \Phi(z)\right|_{\widetilde{\mathcal{S}}_{3}},\left.\quad \operatorname{Res}_{z=0} \widetilde{J}_{2}(z) \Phi(z)\right|_{\widetilde{\mathcal{S}}_{2}}=-\left.\operatorname{Res}_{z=0} \widetilde{J}_{4}(z) \Phi(z)\right|_{\widetilde{\mathcal{S}}_{4}}, \\
& \left.\operatorname{Res}_{z=-1} \widetilde{J}_{1}(z) \Phi(z)\right|_{\widetilde{\mathcal{S}}_{1}}=-\left.\operatorname{Res}_{z=-1} \widetilde{J}_{6}(z) \Phi(z)\right|_{\widetilde{\mathcal{S}}_{6}},\left.\quad \operatorname{Res}_{z=-1} \widetilde{J}_{2}(z) \Phi(z)\right|_{\widetilde{\mathcal{S}}_{2}}=-\left.\operatorname{Res}_{z=-1} \widetilde{J}_{5}(z) \Phi(z)\right|_{\widetilde{\mathcal{S}}_{5}}, \\
& \left.\underset{z=-1 / \tau}{\operatorname{Res}} \widetilde{J}_{1}(z) \Phi(z)\right|_{\widetilde{\mathcal{S}}_{1}}=-\left.\operatorname{Res}_{z=-\mu} \widetilde{J}_{8}(z) \Phi(z)\right|_{\widetilde{\mathcal{S}}_{8}},\left.\underset{z=-1 / \tau}{\operatorname{Res}} \widetilde{J}_{2}(z) \Phi(z)\right|_{\widetilde{\mathcal{S}}_{2}}=-\left.\operatorname{Res}_{z=-\mu} \widetilde{J}_{7}(z) \Phi(z)\right|_{\widetilde{\mathcal{S}}_{7}}, \\
& \left.\operatorname{Res}_{z=-1} \widetilde{J}_{3}(z) \Phi(z)\right|_{\widetilde{\mathcal{S}}_{3}}=-\left.\operatorname{Res}_{z=\mu \tau} \widetilde{J}_{5}(z) \Phi(z)\right|_{\widetilde{\mathcal{S}}_{5}},\left.\quad \operatorname{Res}_{z=-1} \widetilde{J}_{4}(z) \Phi(z)\right|_{\widetilde{\mathcal{S}}_{4}}=-\left.\operatorname{Res}_{z=\mu \tau} \widetilde{J}_{6}(z) \Phi(z)\right|_{\widetilde{\mathcal{S}}_{6}}, \\
& \left.\underset{z=-1 / \tau}{\operatorname{Res}} \widetilde{J}_{3}(z) \Phi(z)\right|_{\widetilde{\mathcal{S}}_{3}}=-\left.\operatorname{Res}_{z=1 / \tau} \widetilde{J}_{7}(z) \Phi(z)\right|_{\widetilde{\mathcal{S}}_{7}},\left.\underset{z=-1 / \tau}{\operatorname{Res}} \widetilde{J}_{4}(z) \Phi(z)\right|_{\widetilde{\mathcal{S}}_{4}}=-\left.\operatorname{Res}_{z=1 / \tau} \widetilde{J}_{8}(z) \Phi(z)\right|_{\widetilde{\mathcal{S}}_{8}} \cdot
\end{aligned}
$$

In the purely massless limit, $m_{1}, \ldots, m_{4} \rightarrow 0$, the kinematic quantities defined above reduce as follows,

$$
\bar{\xi}_{1} \rightarrow 1, \quad \bar{\xi}_{2} \rightarrow 0, \quad \mu \rightarrow 0, \quad \tau \rightarrow \frac{\chi}{1+\chi}, \quad \gamma_{\star}=\frac{1}{16 \chi s_{12}^{3}},
$$

where $\chi \equiv s_{14} / s_{12}$. We point out that the hepta-cut contributions in eqs. (4.21)-(4.24) in ref. [50] after appropriate reparametrization,

$$
\begin{aligned}
X_{2,1,1}^{* *}[1]_{\widetilde{\mathcal{S}}_{5,6}} & =-\frac{1}{16 s_{12}^{3}} \oint \frac{d z}{z(z+\chi)}, \\
X_{2,1,1}^{* *}[1]_{\widetilde{\mathcal{S}}_{7,8}} & =-\frac{1}{16 s_{12}^{3}} \oint \frac{d z}{z(z-\chi-1)}, \\
X_{2,1,1}^{* *}[1]_{\widetilde{\mathcal{S}}_{1,2,3,4}} & =-\frac{1}{16 s_{12}^{3}} \oint \frac{d z}{z(z-\chi)(z-\chi-1)} .
\end{aligned}
$$

\section{$5 \quad$ Master integral projectors}

The hepta-cut localizes the two-loop crossed-box integrand onto a variety of linked Riemann spheres associated with the joint solutions of the on-shell equations. What remains is a one-dimensional complex contour integral whose integrand has poles. We can now choose contours that extract residues of the integrand and effectively obtain an octa-cut such that the integral is completely localized to a point in $\mathbb{C}^{8}$. On top of that, consistency of the unitarity method imposes nontrivial constraints on these contours, however.

The reason is that converting a real slice integral into a multidimensional contour integral in general does not respect various relations among integrals. Each identity leads 
to a constraint which at two loops always can be rearranged and phrased as vanishing of a certain function upon integration over $\mathbb{R}^{D} \times \mathbb{R}^{D}$. Schematically,

$$
X_{2,1,1}^{* *}\left[\Phi\left(\ell_{1}, \ell_{2}\right)\right]=\left.0 \Longrightarrow X_{2,1,1}^{* *}\left[\Phi\left(\ell_{1}, \ell_{2}\right)\right]\right|_{8-\text { cut }}=0
$$

It is easy to understand the nature of these relations if we imagine that we compute an amplitude diagram by diagram. All contractions between loop momenta and external vectors are expressible in terms of the eight fundamental scalar products $\ell_{i} \cdot e_{j}$ for $i=$ 1,2 and $j=1,2,3,4$ where $e=\left(k_{1}, k_{2}, k_{4}, \omega\right)$. Here, $\omega$ is a spurious direction that is perpendicular to the subspace spanned by the four external momenta.

Odd powers of $\ell_{1} \cdot \omega$ and $\ell_{2} \cdot \omega$ vanish upon integration, whereas even powers are reducible in four dimensions. It readily follows that $\ell_{1} \cdot k_{1}, \ell_{1} \cdot k_{2}$ and $\ell_{2} \cdot k_{4}$ can be written in terms of inverse propagators and external invariants. Moreover, $\ell_{2} \cdot k_{2}$ depends linearly on $\ell_{1} \cdot k_{4}$ and $\ell_{2} \cdot k_{1}$. The latter two may be selected as irreducible scalar products. Accordingly, the general numerator polynomial for the problem at hand can be parametrized as follows,

$$
N=\sum_{\left\{\alpha_{1}, \ldots, \alpha_{4}\right\}} c_{\alpha_{1} \cdots \alpha_{4}}\left(\ell_{1} \cdot k_{4}\right)^{\alpha_{1}}\left(\ell_{2} \cdot k_{1}\right)^{\alpha_{2}}\left(\ell_{1} \cdot \omega\right)^{\alpha_{3}}\left(\ell_{2} \cdot \omega\right)^{\alpha_{4}} .
$$

The integrand reduction can be obtained by imposing renormalizability conditions that constrain the exponents of the ISPs and performing the multivariate polynomial division of $N$ modulo a Gröbner basis constructed from the seven inverse propagators. The latter part including identification of ISPs is carried out automatically by the Mathematica package BasisDet [57]. The integrand contains 19 parity-odd and 19 parity-even elements as previously reported [54].

The analysis above suggests that the amplitude contribution in question contains 19 genuine integrals of the form

$$
X_{2,1,1}^{* *}[n, m] \equiv X_{2,1,1}^{* *}\left[\left(\frac{\left(\ell_{1}+k_{4}\right)^{2}}{2}\right)^{n}\left(\frac{\left(\ell_{2}+k_{1}\right)^{2}}{2}\right)^{m}\right] .
$$

Many integrals are expressible as linear combinations of integrals with lower-rank tensors and fewer than seven propagators. This reduction is achieved due to IBP relations that follow from inserting a total derivative into the loop integrand and discarding the boundary term in $D$ dimensions. The relations take the form

$$
X_{2,1,1}^{* *}[n, m]=\sum_{(p, q) \in \text { Basis }} c_{p q} X_{2,1,1}^{* *}[p, q]+\cdots
$$

where $\cdots$ means fewer-propagator topologies that have vanishing hepta-cuts, and the consistency constraint thus reads

$$
\left.X_{2,1,1}^{* *}[n, m]\right|_{8-\mathrm{cut}}=\left.\sum_{(p, q) \in \mathrm{Basis}} c_{p q} X_{2,1,1}^{* *}[p, q]\right|_{8-\mathrm{cut}} .
$$

The IBP relations can be generated by various public computer codes. For this project we used the Mathematica package FIRE [70]. We list below a few examples. There are two 
relations which hold for arbitrary values of the external masses,

$$
\begin{aligned}
X_{2,1,1}^{* *}[0,-1] & =A \cdot X_{2,1,1}^{* *}[0,0]+B \cdot X_{2,1,1}^{* *}[-1,0], \\
X_{2,1,1}^{* *}[-1,-1] & =A \cdot X_{2,1,1}^{* *}[-1,0]+B \cdot X_{2,1,1}^{* *}[-2,0],
\end{aligned}
$$

for $A$ and $B$ given by

$$
A=\frac{1}{4}\left(s_{14}+\frac{m_{1}^{2} m_{3}^{2}-m_{2}^{2} m_{4}^{2}}{s_{12}}\right), \quad B=-\frac{1}{2}\left(1+\frac{m_{1}^{2}-m_{2}^{2}}{s_{12}}\right) .
$$

If for instance $m_{2}=m_{3}=m_{4}=0$ and $m_{1}$ is nonzero, then there is one additional relation among these integrals,

$$
X_{2,1,1}^{* *}[-2,0]=-\frac{s_{12}^{2} s_{14}}{16\left(m_{1}^{2}-s_{12}\right)} X_{2,1,1}^{* *}[0,0]-\frac{1}{8}\left(3+\frac{2 s_{14}}{m_{1}^{2}-s_{12}}\right) X_{2,1,1}^{* *}[-1,0] .
$$

The parity-odd terms in the integrand basis of course vanish identically after the loop momentum integration has been performed, but they are nonetheless very important for integrand-level reduction and unitarity purposes. It is sufficient to require that the full variety of integrals with Levi-Civita insertions, after invoking momentum conservation,

$$
\varepsilon\left(\ell_{1}, k_{2}, k_{3}, k_{4}\right), \quad \varepsilon\left(\ell_{2}, k_{2}, k_{3}, k_{4}\right), \quad \varepsilon\left(\ell_{1}, \ell_{2}, k_{1}, k_{2}\right), \quad \varepsilon\left(\ell_{1}, \ell_{2}, k_{1}, k_{3}\right), \quad \varepsilon\left(\ell_{1}, \ell_{2}, k_{2}, k_{3}\right),
$$

continue to integrate to zero on general contours in $\mathbb{C}^{4} \times \mathbb{C}^{4}$.

Our goal of the rest of this paper is to massage the amplitude master equation (2.1) into a form that allows us to project the master integral coefficients.

\subsection{One-mass projectors}

The simplest configuration is the one-mass diagram with, say, $m_{1} \neq 0$. As in the purely massless case [50,54], there are two master integrals so the amplitude contribution can be expressed as

$$
\mathcal{A}_{4}^{(2)}=c_{1} X_{2,1,1}^{* *}[1]+c_{2} X_{2,1,1}^{* *}\left[\left(\ell_{1}+k_{4}\right)^{2} / 2\right]+\cdots,
$$

where integrals with less than seven propagators are truncated. Since momentum $k_{4}$ is massless, we have $\ell_{1} \cdot k_{4}=\left(\ell_{1}+k_{4}\right)^{2} / 2$. We decide to encircle the following set of global poles,

$$
\left\{\widetilde{\mathcal{G}}_{i}\right\}=\left(\widetilde{\mathcal{G}}_{1 \cap 6}, \widetilde{\mathcal{G}}_{1 \cap 8}, \widetilde{\mathcal{G}}_{3 \cap 5}, \widetilde{\mathcal{G}}_{3 \cap 7}, \widetilde{\mathcal{G}}_{1 \cap 3}, \widetilde{\mathcal{G}}_{2 \cap 5}, \widetilde{\mathcal{G}}_{2 \cap 7}, \widetilde{\mathcal{G}}_{4 \cap 6}, \widetilde{\mathcal{G}}_{4 \cap 8}, \widetilde{\mathcal{G}}_{2 \cap 4}\right) \equiv\left(\widetilde{\mathcal{G}}_{1}, \ldots, \widetilde{\mathcal{G}}_{10}\right),
$$

and let $\widetilde{\boldsymbol{\Omega}}$ denote the corresponding weights,

$$
\boldsymbol{\Omega}^{\prime}=\left(\widetilde{\omega}_{1 \cap 6}, \widetilde{\omega}_{1 \cap 8}, \widetilde{\omega}_{3 \cap 5}, \widetilde{\omega}_{3 \cap 7}, \widetilde{\omega}_{1 \cap 3}, \widetilde{\omega}_{2 \cap 5}, \widetilde{\omega}_{2 \cap 7}, \widetilde{\omega}_{4 \cap 6}, \widetilde{\omega}_{4 \cap 8}, \widetilde{\omega}_{2 \cap 4}\right) \equiv\left(\widetilde{\omega}_{1}, \ldots, \widetilde{\omega}_{10}\right) .
$$


The hepta-cut two-loop crossed box integrals reduce to

$$
\begin{aligned}
& X_{2,1,1}^{* *}[1]_{\widetilde{\mathcal{S}}_{1,2}} \longrightarrow-\frac{m_{1}^{2}-s_{12}}{\chi s_{12}} \oint \frac{d z}{z(z+1)\left(z+\left(s_{12}(1+\chi)-m_{1}^{2}\right) /\left(\chi s_{12}\right)\right)}, \\
& X_{2,1,1}^{* *}[1]_{\widetilde{\mathcal{S}}_{3,4}} \longrightarrow+\frac{m_{1}^{2}-s_{12}}{\chi s_{12}} \oint \frac{d z}{z(z+1)\left(z+\left(s_{12}(1+\chi)-m_{1}^{2}\right) /\left(\chi s_{12}\right)\right)},
\end{aligned}
$$

and likewise for the remaining four solutions, which we do not explicitly need here. Notice that we stripped off the overall factor. As expected we immediately derive five linearly independent contour constraints arising from parity-odd numerator insertions,

$$
\left(\begin{array}{rrrrrrrrrr}
1 & 0 & 0 & 0 & 0 & -1 & 0 & 0 & 0 & 0 \\
0 & 1 & 0 & 0 & 0 & 0 & -1 & 0 & 0 & 0 \\
0 & 0 & 1 & 0 & 0 & 0 & 0 & -1 & 0 & 0 \\
0 & 0 & 0 & 1 & 0 & 0 & 0 & 0 & -1 & 0 \\
0 & 0 & 0 & 0 & 1 & 0 & 0 & 0 & 0 & -1
\end{array}\right)\left(\begin{array}{c}
\widetilde{\omega}_{1 \cap 6} \\
\widetilde{\omega}_{1 \cap 8} \\
\widetilde{\omega}_{3 \cap 5} \\
\widetilde{\omega}_{3 \cap 7} \\
\widetilde{\omega}_{1 \cap 3} \\
\widetilde{\omega}_{2 \cap 5} \\
\widetilde{\omega}_{2 \cap 7} \\
\widetilde{\omega}_{4 \cap 6} \\
\widetilde{\omega}_{4 \cap 8} \\
\widetilde{\omega}_{2 \cap 4}
\end{array}\right)=0 .
$$

Moreover, there are three linearly independent IBP constraints,

$$
\begin{array}{r}
\widetilde{\boldsymbol{\Omega}} \cdot(0,1,0,1,0,0,1,0,1,0)=0, \\
\widetilde{\boldsymbol{\Omega}} \cdot(1,-1,1,-1,0,1,-1,1,-1,0)=0, \\
\widetilde{\boldsymbol{\Omega}} \cdot(1,1,-1,-1,2,1,1,-1,-1,2)=0 .
\end{array}
$$

The residues computed by the master integrals around the global poles are

$$
\begin{aligned}
\operatorname{Res}_{\left\{\widetilde{\mathcal{G}}_{i}\right\}} X_{2,1,1}^{* *}[1]= & -1,-\frac{\chi s_{12}}{m_{1}^{2}-(1+\chi) s_{12}}, 1, \frac{\chi s_{12}}{m_{1}^{2}-(1+\chi) s_{12}}, \frac{\chi s_{12}}{m_{1}^{2}-(1+\chi) s_{12}}, \\
& \left.-1,-\frac{\chi s_{12}}{m_{1}^{2}-(1+\chi) s_{12}}, 1, \frac{\chi s_{12}}{m_{1}^{2}-(1+\chi) s_{12}}, \frac{\chi s_{12}}{m_{1}^{2}-(1+\chi) s_{12}}\right), \\
\operatorname{Res}_{\left\{\widetilde{\mathcal{G}}_{i}\right\}} X_{2,1,1}^{* *}\left[\ell_{1} \cdot k_{4}\right]= & \frac{\chi s_{12}^{2}}{2\left(m_{1}^{2}-(1+\chi) s_{12}\right)}(0,1,0,-1,-1,0,1,0,-1,-1) .
\end{aligned}
$$

We exploit the freedom to choose contours after imposing the reduction conditions and define two master integral projectors (also called master contours) which extract of either of the master integral coefficients,

$$
\begin{aligned}
& \mathcal{M}_{1} \cdot\left(\underset{\left\{\widetilde{\mathcal{G}}_{i}\right\}}{\operatorname{Res}} X_{2,1,1}^{* *}[1], \underset{\left\{\widetilde{\mathcal{G}}_{i}\right\}}{\operatorname{Res}} X_{2,1,1}^{* *}\left[\ell_{1} \cdot k_{4}\right]\right)=(1,0), \\
& \mathcal{M}_{2} \cdot\left(\underset{\left\{\widetilde{\mathcal{G}}_{i}\right\}}{\operatorname{Res}} X_{2,1,1}^{* *}[1], \underset{\left\{\widetilde{\mathcal{G}}_{i}\right\}}{\operatorname{Res}} X_{2,1,1}^{* *}\left[\ell_{1} \cdot k_{4}\right]\right)=(0,1) .
\end{aligned}
$$


Here, $\mathcal{M}_{1}$ and $\mathcal{M}_{2}$ are just particular lists of the winding numbers of the corresponding global poles with the property that they only receive contribution from one master integral, which is also normalized to unity.

The eight contour constraints together with either of the projectors are in practice arranged as $10 \times 10$ matrices. The rank is 10 and the solutions for the weights are uniquely determined. We find that the projectors are characterized by the 10-tuples

$$
\begin{aligned}
\mathcal{M}_{1}=\frac{1}{16}(-3, & 1,3,-1,2,-3,1,3,-1,2) \\
\mathcal{M}_{2}=\frac{1}{8 \chi s_{12}^{2}} & \left(-m_{1}^{2}+(1-2 \chi) s_{12}, 3 m_{1}^{2}-(3+2 \chi) s_{12}, m_{1}^{2}-(1-2 \chi) s_{12}\right. \\
& -3 m_{1}^{2}+(3+2 \chi) s_{12}, 2\left(-m_{1}^{2}+(1+2 \chi) s_{12}\right),-m_{1}^{2}+(1-2 \chi) s_{12} \\
& 3 m_{1}^{2}-(3+2 \chi) s_{12}, m_{1}^{2}-(1-2 \chi) s_{12},-3 m_{1}^{2}+(3+\chi) s_{12} \\
& \left.2\left(-m_{1}^{2}+(1+2 \chi) s_{12}\right)\right)
\end{aligned}
$$

The master integral coefficients can be written compactly in terms of tree-level data as

$$
c_{i}=\oint_{\mathcal{M}_{i}} d z \widetilde{J}(z) \sum_{\substack{\text { helicities } \\ \text { particles }}} \prod_{k=1}^{6} A_{(k)}^{\text {tree }}(z),
$$

where the rescaled Jacobian for this configuration is defined by

$$
\widetilde{J}(z) \equiv \pm \frac{m_{1}^{2}-s_{12}}{\chi s_{12}} \frac{1}{z(z+1)\left(z+\left(s_{12}(1+\chi)-m_{1}^{2}\right) /\left(\chi s_{12}\right)\right.} .
$$

The computation of the remaining one-mass configurations is essentially equivalent to the one described here and the projectors are similar. The lack of symmetry in the two-loop crossed box suggests that we also derive projectors for the one-mass diagram with $m_{4} \neq 0$ (or $m_{3} \neq 0$ ). The hepta-cuts in the limit $m_{1}, m_{2}, m_{3} \rightarrow 0$ follow from eqs. (4.21)-(4.24),

$$
\begin{aligned}
& X_{2,1,1}^{* *}[1]_{\widetilde{\mathcal{S}}_{1,2}} \longrightarrow+\frac{1}{\chi} \oint \frac{d z}{z(z+1)\left(z+\left(s_{12}(1+\chi)-m_{4}^{2}\right) /\left(\chi s_{12}\right)\right)}, \\
& X_{2,1,1}^{* *}[1]_{\widetilde{\mathcal{S}}_{3,4}} \longrightarrow-\frac{1}{\chi} \oint \frac{d z}{z(z+1)\left(z+\left(s_{12}(1+\chi)-m_{4}^{2}\right) /\left(\chi s_{12}\right)\right)},
\end{aligned}
$$

whereas the singular point locus and parity-odd vanishing constraints carry over directly from the calculation above. The residues in the masters are

$$
\begin{aligned}
& \operatorname{Res}_{\left\{\widetilde{\mathcal{G}}_{i}\right\}} X_{2,1,1}^{* *}[1]= \\
& \frac{s_{12}}{\left(m_{4}^{2}-s_{12}\right)\left(m_{4}^{2}-(1+\chi) s_{12}\right)}\left(m_{4}^{2}-(1+\chi) s_{12}, \chi s_{12},(1+\chi) s_{12}-m_{4}^{2},\right. \\
& \left.\quad-\chi s_{12}, s_{12}-m_{4}^{2}, m_{4}^{2}-(1+\chi) s_{12}, \chi s_{12},(1+\chi) s_{12}-m_{4}^{2},-\chi s_{12}, s_{12}-m_{4}^{2}\right),
\end{aligned}
$$$$
\operatorname{Res}_{\left\{\widetilde{\mathcal{G}}_{i}\right\}} X_{2,1,1}^{* *}\left[\left(\ell_{1}+k_{4}\right)^{2} / 2\right]=\frac{\chi s_{12}^{2}}{2\left(m_{4}^{2}-(1+\chi) s_{12}\right)}(0,1,0,-1,-1,0,1,0,-1,-1),
$$ 
and the unique projectors read

$$
\begin{aligned}
\mathcal{M}_{1}=\frac{m_{4}^{2}-s_{12}}{16 s_{12}}(3,-1,-3,1,-2,3,-1,-3,1,-2) & \\
\mathcal{M}_{2}=\frac{1}{8 \chi s_{12}^{2}} & \left((1-2 \chi) s_{12}-m_{4}^{2}, 3 m_{4}^{2}-(3+2 \chi) s_{12}, m_{4}^{2}-(1-2 \chi) s_{12}\right. \\
& (3+2 \chi) s_{12}-3 m_{4}^{2}, 2\left((1+2 \chi) s_{12}-m_{4}^{2}\right),(1-2 \chi) s_{12}-m_{4}^{2} \\
& 3 m_{4}^{2}-(3+2 \chi) s_{12}, m_{4}^{2}-(1-2 \chi) s_{12},(3+2 \chi) s_{12}-3 m_{4}^{2}, \\
& \left.2\left((1+2 \chi) s_{12}-m_{4}^{2}\right)\right) .
\end{aligned}
$$

These master contours respect the three linearly independent IBP constraints

$$
\begin{array}{r}
\widetilde{\boldsymbol{\Omega}} \cdot(0,1,0,1,0,0,1,0,1,0)=0, \\
\widetilde{\boldsymbol{\Omega}} \cdot(1,-1,1,-1,0,1,-1,1,-1,0)=0, \\
\widetilde{\boldsymbol{\Omega}} \cdot(1,1,-1,-1,2,1,1,-1,-1,2)=0 .
\end{array}
$$

The expressions for the one-mass projectors derived here are consistent with the purely massless calculation reported in ref. [50].

\subsection{Two-mass projectors}

As previously explained, there are four kinematically inequivalent distributions of massless and massive external legs in the two-mass four-point crossed box. Indeed, we distinguish between the two-mass short-side diagrams with either both massive legs situated in the planar or nonplanar end and the long-side and diagonal diagrams. From the point of view of the global structure of the hepta-cut, the latter three are similar and can be treated within the regime of the octuply pinched genus-3 curve whereas the first diagram is a variant of the three- and four-mass case.

The long-side two-mass diagram can be studied by taking over the singular point locus (5.12), basis integral decomposition as well as the parity-odd contour constraints (5.15) it turns out. We assume that $m_{1} m_{4} \neq 0$ and $m_{2}=m_{3}=0$. Under these circumstances, the relevant hepta-cuts are

$$
\begin{aligned}
& X_{2,1,1}^{* *}[1]_{\widetilde{\mathcal{S}}_{1,2}} \longrightarrow+\frac{s_{12}-m_{1}^{2}}{\chi s_{12}} \oint \frac{d z}{z(z+1)(z+\lambda)}, \\
& X_{2,1,1}^{* *}[1]_{\widetilde{\mathcal{S}}_{3,4}} \longrightarrow-\frac{s_{12}-m_{1}^{2}}{\chi s_{12}} \oint \frac{d z}{z(z+1)(z+\lambda)},
\end{aligned}
$$

where the pole location $\lambda$ is defined by

$$
\lambda \equiv \frac{m_{1}^{2}\left(m_{4}^{2}-s_{12}\right)+s_{12}\left(s_{12}(1+\chi)-m_{4}^{2}\right)}{\chi s_{12}^{2}} .
$$

These hepta-cuts clearly coincide with eqs. (5.13)-(5.14) and (5.26)-(5.27) in the respective limits, $m_{4} \rightarrow 0$ and $m_{1} \rightarrow 0$. 
The residues associated with the two master integrals are as follows,

$$
\begin{aligned}
\operatorname{Res}_{\left\{\widetilde{\mathcal{G}}_{i}\right\}} X_{2,1,1}^{* *}[1] & =N_{1}\left(r_{1}, r_{2},-r_{1},-r_{2}, r_{3}, r_{1}, r_{2},-r_{1},-r_{2}, r_{3}\right), \\
\operatorname{Res}_{\left\{\widetilde{\mathcal{G}}_{i}\right\}} X_{2,1,1}^{* *}\left[\left(\ell_{1}+k_{4}\right)^{2} / 2\right] & =N_{2}(0,-1,0,1,1,0,-1,0,1,1),
\end{aligned}
$$

where $N_{1}$ and $N_{2}$ are given by

$$
\begin{aligned}
& N_{1} \equiv \frac{s_{12}}{\left(m_{4}^{2}-s_{12}\right)\left(\left(m_{1}^{2}+m_{4}^{2}\right) s_{12}-(1+\chi) s_{12}^{2}-m_{1}^{2} m_{4}^{2}\right)}, \\
& N_{2} \equiv \frac{\chi s_{12}^{3}}{2\left(m_{1}^{2}-s_{12}\right)\left(m_{4}^{2}-s_{12}\right)+2 \chi s_{12}^{2}},
\end{aligned}
$$

and the $r_{i}$ s are defined as

$$
r_{1} \equiv s_{12}\left(m_{4}^{2}-(1+\chi) s_{12}\right)-m_{1}^{2}\left(m_{4}^{2}-s_{12}\right), \quad r_{2} \equiv \chi s_{12}^{2}, \quad r_{3} \equiv\left(m_{1}^{2}-s_{12}\right)\left(m_{4}^{2}-s_{12}\right) .
$$

The projectors for the coefficients $c_{1}$ and $c_{2}$ become

$$
\begin{aligned}
& \mathcal{M}_{1}=\frac{m_{4}^{2}-s_{12}}{16 s_{12}}(3,-1,-3,1,-2,3,-1,-3,1,-2), \\
& \mathcal{M}_{2}=\frac{1}{8 \chi s_{12}^{3}}\left(q_{1}, q_{2},-q_{1},-q_{2}, q_{3}, q_{1}, q_{2},-q_{1},-q_{2}, q_{3}\right),
\end{aligned}
$$

for constants $q_{1}, q_{2}$ and $q_{3}$ where

$$
\begin{aligned}
& q_{1} \equiv+m_{1}^{2}\left(m_{4}^{2}-s_{12}\right)-s_{12}\left(m_{4}^{2}-(1-2 \chi) s_{12}\right) \\
& q_{2} \equiv-2 \chi s_{12}^{2}-3\left(m_{1}^{2}-s_{12}\right)\left(m_{4}^{2}-s_{12}\right) \\
& q_{3} \equiv+2\left(2 \chi s_{12}^{2}+\left(m_{1}^{2}-s_{12}\right)\left(m_{4}^{2}-s_{12}\right)\right)
\end{aligned}
$$

For this external kinematics, the three independent consistency relations from IBP identities are

$$
\begin{array}{r}
\widetilde{\boldsymbol{\Omega}} \cdot(0,1,0,1,0,0,1,0,1,0)=0, \\
\widetilde{\boldsymbol{\Omega}} \cdot(1,-1,1,-1,0,1,-1,1,-1,0)=0, \\
\widetilde{\boldsymbol{\Omega}} \cdot(1,1,-1,-1,2,1,1,-1,-1,2)=0 .
\end{array}
$$

We have also derived projectors for the two-mass diagonal configuration. The computation essentially resembles that of the two-mass long-side diagram, meaning that the same singular point locus, integral basis and contour constraints can be used. For $m_{2}=m_{4}=0$ and $m_{1} m_{3} \neq 0$, the hepta-cuts evaluated at the branches $\mathcal{S}_{1}, \ldots, \mathcal{S}_{4}$ are

$$
\begin{aligned}
& X_{2,1,1}^{* *}[1]_{\widetilde{\mathcal{S}}_{1,2}} \longrightarrow+\frac{\left(m_{1}^{2}-s_{12}\right)\left(m_{3}^{2}-s_{12}\right)}{\chi s_{12}^{2}-m_{1}^{2} m_{3}^{2}} \oint \frac{d z}{z(z+1)(z+\lambda)}, \\
& X_{2,1,1}^{* *}[1]_{\widetilde{\mathcal{S}}_{3,4}} \longrightarrow-\frac{\left(m_{1}^{2}-s_{12}\right)\left(m_{3}^{2}-s_{12}\right)}{\chi s_{12}^{2}-m_{1}^{2} m_{3}^{2}} \oint \frac{d z}{z(z+1)(z+\lambda)},
\end{aligned}
$$


where the third pole $\lambda$ is now defined by

$$
\lambda \equiv \frac{s_{12}\left(m_{1}^{2}+m_{3}^{2}-(1+\chi) s_{12}\right)}{m_{1}^{2} m_{3}^{2}-\chi s_{12}^{2}} .
$$

It can be shown that the master contours for this kind of integrals are characterized by the following numbers,

$$
\begin{aligned}
& \mathcal{M}_{1}=\frac{1}{16}(3,-1,-3,1,-2,3,-1,-3,1,-2), \\
& \mathcal{M}_{2}=\frac{1}{8\left(m_{3}^{2}-s_{12}\right)\left(m_{1}^{2} m_{3}^{2}-\chi s_{12}^{2}\right)}\left(q_{1}, q_{2},-q_{1},-q_{2}, q_{3}, q_{1}, q_{2},-q_{1},-q_{2}, q_{3}\right),
\end{aligned}
$$

and the three independent weights $q_{1}, q_{2}$ and $q_{3}$ in $\mathcal{M}_{2}$ are

$$
\begin{aligned}
& q_{1} \equiv+m_{1}^{2}\left(3 m_{3}^{2}-s_{12}\right)-s_{12}\left(m_{3}^{2}-(1-2 \chi) s_{12}\right), \\
& q_{2} \equiv-m_{1}^{2}\left(m_{3}^{2}-3 s_{12}\right)+s_{12}\left(3 m_{3}^{2}-(3+2 \chi) s_{12}\right), \\
& q_{3} \equiv-2\left(m_{1}^{2}\left(m_{3}^{2}+s_{12}\right)+s_{12}\left(m_{3}^{2}-(1+2 \chi) s_{12}\right)\right) .
\end{aligned}
$$

Our next example is the short-side two-mass diagram with $m_{1} m_{2} \neq 0$ and $m_{3}=m_{4}=0$ which is a smooth limit of the three- and four-mass case. Accordingly, we now have the master equation

$$
\mathcal{A}_{4}^{(2)}=c_{1} X_{2,1,1}^{* *}[1]+c_{2} X_{2,1,1}^{* *}\left[\left(\ell_{1}+k_{4}\right)^{2} / 2\right]+c_{3} X_{2,1,1}^{* *}\left[\left(\left(\ell_{1}+k_{4}\right)^{2} / 2\right)^{2}\right]+\cdots,
$$

and we must choose a new singular point locus for this computation. It is natural to encircle the following ten global poles,

$$
\left\{\mathcal{G}_{i}\right\}=\left(\mathcal{G}_{1 \cap 4}, \mathcal{G}_{1 \cap 6}, \mathcal{G}_{1 \cap 3}, \mathcal{G}_{1 \cap 5}, \mathcal{G}_{1, \infty_{L R}}, \mathcal{G}_{2 \cap 3}, \mathcal{G}_{2 \cap 5}, \mathcal{G}_{2 \cap 4}, \mathcal{G}_{2 \cap 6}, \mathcal{G}_{2, \infty_{L R}}\right) \equiv\left(\mathcal{G}_{1}, \ldots, \mathcal{G}_{10}\right)
$$

We remind that $\left(\mathcal{G}_{1}, \ldots, \mathcal{G}_{5}\right)$ and $\left(\mathcal{G}_{5}, \ldots, \mathcal{G}_{10}\right)$, are located at the following values of $z$ on the Riemann sphere parametrized by $\mathcal{S}_{1}$ and $\mathcal{S}_{2}$ respectively,

$$
\left\{-1,-\frac{1}{\tau},-\frac{\bar{\xi}_{2}}{\bar{\xi}_{1}},-\frac{\tau \bar{\xi}_{2}}{\bar{\xi}_{1}}, 0\right\}
$$

Evidently, on-shell branches three through six are eliminated and we retain only the following two hepta-cut integrals,

$$
X_{2,1,1}^{* *}[1]_{\mathcal{S}_{1,2}} \longrightarrow+N \oint d z \frac{z}{(z+1)(z+1 / \tau)\left(z+\bar{\xi}_{2} / \bar{\xi}_{1}\right)\left(z+\tau \bar{\xi}_{2} / \bar{\xi}_{1}\right)},
$$

where the overall constant is given by

$$
N \equiv \frac{\left(\gamma_{12}^{2}-m_{1}^{2} m_{2}^{2}\right)^{3}}{\gamma_{12}^{3}\left(\gamma_{12}+m_{2}^{2}\right)\left(m_{1}^{2} m_{2}^{2}+\chi \gamma_{12} s_{12}\right)},
$$

and the poles are located at

$$
1 / \tau=\frac{\gamma_{12}\left(\gamma_{12}+\chi s_{12}\right)}{m_{1}^{2} m_{2}^{2}+\chi \gamma_{12} s_{12}}, \quad \frac{\bar{\xi}_{2}}{\bar{\xi}_{1}}=\frac{m_{1}^{2} m_{2}^{2}\left(\gamma_{12}+\chi s_{12}\right)}{\gamma_{12}\left(m_{1}^{2} m_{2}^{2}+\chi \gamma_{12} \chi_{12}\right)}, \quad \frac{\tau \bar{\xi}_{2}}{\bar{\xi}_{1}}=\frac{m_{1}^{2} m_{2}^{2}}{\gamma_{12}^{2}} .
$$


Requiring that all parity-odd numerator insertions have vanishing hepta-cuts translates into the statement

$$
\left(\begin{array}{rrrrrrrrrr}
1 & 0 & 0 & 0 & 0 & -1 & 0 & 0 & 0 & 0 \\
0 & 1 & 0 & 0 & 0 & 0 & -1 & 0 & 0 & 0 \\
0 & 0 & 1 & 0 & 0 & 0 & 0 & -1 & 0 & 0 \\
0 & 0 & 0 & 1 & 0 & 0 & 0 & 0 & -1 & 0 \\
0 & 0 & 0 & 0 & 1 & 0 & 0 & 0 & 0 & -1
\end{array}\right)\left(\begin{array}{c}
\omega_{1 \cap 4} \\
\omega_{1 \cap 6} \\
\omega_{1 \cap 3} \\
\omega_{1 \cap 5} \\
\omega_{1, \infty_{L R}} \\
\omega_{2 \cap 3} \\
\omega_{2 \cap 5} \\
\omega_{2 \cap 4} \\
\omega_{2 \cap 6} \\
\omega_{2, \infty_{L R}}
\end{array}\right)=0
$$

There are two linearly independent constraints from IBP relations,

$$
\begin{array}{r}
\boldsymbol{\Omega} \cdot(0,1,0,1,-1,0,1,0,1,-1)=0, \\
\boldsymbol{\Omega} \cdot(1,-1,1,-1,0,1,-1,1,-1,0)=0 .
\end{array}
$$

Resolving the contour constraints leaves three contour weights undetermined, exactly balancing the number of master integrals for this problem. The master contours which pick up contribution from one basis integral and annihilate the other two are,

$$
\begin{gathered}
\mathcal{M}_{1} \cdot\left(\underset{\left\{\mathcal{G}_{i}\right\}}{\operatorname{Res}} X_{2,1,1}^{* *}[1], \underset{\left\{\mathcal{G}_{i}\right\}}{\operatorname{Res}} X_{2,1,1}^{* *}\left[\left(\ell_{1}+k_{4}\right)^{2} / 2\right], \underset{\left\{\mathcal{G}_{i}\right\}}{\operatorname{Res}} X_{2,1,1}^{* *}\left[\left(\left(\ell_{1}+k_{4}\right)^{2} / 2\right)^{2}\right]\right)=(1,0,0), \\
\mathcal{M}_{2} \cdot\left(\underset{\left\{\mathcal{G}_{i}\right\}}{\operatorname{Res}} X_{2,1,1}^{* *}[1], \underset{\left\{\mathcal{G}_{i}\right\}}{\operatorname{Res}} X_{2,1,1}^{* *}\left[\left(\ell_{1}+k_{4}\right)^{2} / 2\right], \underset{\left\{\mathcal{G}_{i}\right\}}{\operatorname{Res}} X_{2,1,1}^{* *}\left[\left(\left(\ell_{1}+k_{4}\right)^{2} / 2\right)^{2}\right]\right)=(0,1,0), \\
\mathcal{M}_{3} \cdot\left(\underset{\left\{\mathcal{G}_{i}\right\}}{\operatorname{Res}} X_{2,1,1}^{* *}[1], \underset{\left\{\mathcal{G}_{i}\right\}}{\operatorname{Re}} X_{2,1,1}^{* *}\left[\left(\ell_{1}+k_{4}\right)^{2} / 2\right], \underset{\left\{\mathcal{G}_{i}\right\}}{\operatorname{Re}} X_{2,1,1}^{* *}\left[\left(\left(\ell_{1}+k_{4}\right)^{2} / 2\right)^{2}\right]\right)=(0,0,1) .
\end{gathered}
$$

After changing the remaining one-dimensional contour into a linear combination of small circles around the global poles, the three master integrals reduce to the following residues,

$$
\begin{aligned}
\operatorname{Res}_{\left\{\mathcal{G}_{i}\right\}} X_{2,1,1}^{* *}[1] & =N_{1}\left(r_{1},-r_{2},-r_{1}, r_{2}, 0, r_{1},-r_{2},-r_{1}, r_{2}, 0\right), \\
\operatorname{Res}_{\left\{\mathcal{G}_{i}\right\}} X_{2,1,1}^{* *}\left[\left(\ell_{1}+k_{4}\right)^{2} / 2\right] & =N_{2}(0,-1,0,1,0,0,-1,0,1,0), \\
\operatorname{Res}_{\left\{\mathcal{G}_{i}\right\}} X_{2,1,1}^{* *}\left[\left(\left(\ell_{1}+k_{4}\right)^{2} / 2\right)^{2}\right] & =N_{3}\left(0, r_{3}, 0,-r_{3}, r_{1}, 0, r_{3}, 0,-r_{3}, r_{1}\right),
\end{aligned}
$$

where

$$
\begin{aligned}
& N_{1} \equiv-\frac{m_{1}^{2} m_{2}^{2}+\chi \gamma_{12} s_{12}}{\chi s_{12}\left(\gamma_{12}+m_{2}^{2}\right)\left(\gamma_{12}^{2}+m_{1}^{2} m_{2}^{2}+\chi \gamma_{12} s_{12}\right)}, \\
& N_{2} \equiv+\frac{\left(\gamma_{12}+m_{1}^{2}\right)\left(m_{1}^{2} m_{2}^{2}+\chi \gamma_{12} s_{12}\right)}{2\left(\gamma_{12}^{2}+m_{1}^{2} m_{2}^{2}+\chi \gamma_{12} s_{12}\right)} \\
& N_{3} \equiv+\frac{\left(\gamma_{12}+m_{1}^{2}\right)^{2}\left(\gamma_{12}+m_{2}^{2}\right)\left(m_{1}^{2} m_{2}^{2}+\chi \gamma_{12} s_{12}\right)}{4 \gamma_{12}\left(\gamma_{12}^{2}-m_{1}^{2} m_{2}^{2}\right)\left(\gamma_{12}^{2}+m_{1}^{2} m_{2}^{2}+\chi \gamma_{12} s_{12}\right)}
\end{aligned}
$$


and

$$
r_{1} \equiv \gamma_{12}^{2}+m_{1}^{2} m_{2}^{2}+\chi \gamma_{12} s_{12}, \quad r_{2} \equiv \chi \gamma_{12} s_{12}, \quad r_{3} \equiv \gamma_{12}^{2}-m_{1}^{2} m_{2}^{2}
$$

The projectors for the integral coefficients take the form

$$
\begin{aligned}
& \mathcal{M}_{1}=\frac{\left(\gamma_{12}+m_{2}^{2}\right) \chi s_{12}}{4\left(m_{1}^{2} m_{2}^{2}+\gamma_{12} \chi s_{12}\right)}(-1,0,1,0,0,-1,0,1,0,0), \\
& \mathcal{M}_{2}=\frac{1}{2\left(\gamma_{12}+m_{1}^{2}\right)\left(m_{1}^{2} m_{2}^{2}+\gamma_{12} \chi s_{12}\right)}\left(q_{1}, q_{2}, q_{3}, q_{4}, q_{5}, q_{1}, q_{2}, q_{3}, q_{4}, q_{5}\right), \\
& \mathcal{M}_{3}=\frac{\gamma_{12}\left(\gamma_{12}^{2}-m_{1}^{2} m_{2}^{2}\right)}{\left(\gamma_{12}+m_{1}^{2}\right)^{2}\left(\gamma_{12}+m_{2}^{2}\right)\left(m_{1}^{2} m_{2}^{2}+\gamma_{12} \chi s_{12}\right)}(1,1,1,1,2,1,1,1,1,2) .
\end{aligned}
$$

where $q_{1}, \ldots, q_{5}$ are defined by

$$
\begin{aligned}
& q_{1} \equiv \gamma_{12}^{2}-m_{1}^{2} m_{2}^{2}-\gamma_{12} \chi s_{12}, \quad q_{2} \equiv-2 m_{1}^{2} m_{2}^{2}-\gamma_{12} \chi s_{12}, \\
& q_{3} \equiv \gamma_{12}^{2}-m_{1}^{2} m_{2}^{2}+\gamma_{12} \chi s_{12}, \quad q_{4} \equiv q_{1}-q_{2}+q_{3}, \quad q_{5} \equiv q_{1}+q_{3} \text {. }
\end{aligned}
$$

We point out that although the projectors are functions of irrational quantities such as $\gamma_{12}$ which has a square root, the final integral coefficients obtained in this way are rational in external invariants.

\subsection{Three-mass projectors}

The three-mass case with $m_{1}=0$ or $m_{2}=0$ and the remaining three external masses nonzero is similar to the two-mass long-side calculation previously presented. Here we will instead focus on the three-mass diagram with $m_{4}=0$ which has the two-mass short-side diagram with $m_{1} m_{2} \neq 0$ as a smooth limit. This means that we can continue to use the integral basis (5.57), the singular point locus (5.58) and the parity-odd constraints (5.63). Moreover, the contour constraints from IBP relations are identical to those in eqs. (5.64)(5.65).

For this problem, we will use the hepta-cuts for $\mathcal{S}_{1}$ and $\mathcal{S}_{2}$ from eq. (4.14) with $\mu=0$ and without the overall factor of $\gamma_{\star}$,

$$
X_{2,1,1}^{* *}[1]_{\mathcal{S}_{1,2}} \longrightarrow \frac{(1-\tau)\left(\bar{\xi}_{1}-\tau \bar{\xi}_{2}\right)}{\tau \bar{\xi}_{1}^{2}} \oint d z \frac{z}{(z+1)(z+1 / \tau)\left(z+\bar{\xi}_{2} / \bar{\xi}_{1}\right)\left(z+\tau \bar{\xi}_{2} / \bar{\xi}_{1}\right)}
$$

It is possible to obtain rather clean forms of the residues computed by the hepta-cut master integrals at the singular point locus, if we prefer quantities constructed from flat momenta, $\bar{\xi}_{1}, \bar{\xi}_{2}$ and $\tau$, instead of the usual Mandelstam variables and external masses. Indeed, the 
residues can be expressed as

$$
\begin{aligned}
\operatorname{Res}_{\left\{\mathcal{G}_{i}\right\}} X_{2,1,1}^{* *}[1]= & -\frac{1}{\bar{\xi}_{1}-\bar{\xi}_{2}}, \frac{\tau}{\bar{\xi}_{1}-\tau^{2} \bar{\xi}_{2}}, \frac{1}{\bar{\xi}_{1}-\bar{\xi}_{2}},-\frac{\tau}{\bar{\xi}_{1}-\tau^{2} \bar{\xi}_{2}}, 0, \\
& \left.-\frac{1}{\bar{\xi}_{1}-\bar{\xi}_{2}}, \frac{\tau}{\bar{\xi}_{1}-\tau^{2} \bar{\xi}_{2}}, \frac{1}{\bar{\xi}_{1}-\bar{\xi}_{2}},-\frac{\tau}{\bar{\xi}_{1}-\tau^{2} \bar{\xi}_{2}}, 0\right), \quad \\
\operatorname{Res}_{\left\{\mathcal{G}_{i}\right\}} X_{2,1,1}^{* *}\left[\left(\ell_{1}+k_{4}\right)^{2} / 2\right]= & \left(0,-\frac{(1-\tau)\left(\bar{\xi}-\tau \bar{\xi}_{2}\right) k_{1}^{b} \cdot k_{4}^{b}}{\bar{\xi}_{1}-\tau^{2} \bar{\xi}_{2}}, 0, \frac{(1-\tau)\left(\bar{\xi}-\tau \bar{\xi}_{2}\right) k_{1}^{b} \cdot k_{4}^{b}}{\bar{\xi}_{1}-\tau^{2} \bar{\xi}_{2}}, 0,\right. \\
& \left.0,-\frac{(1-\tau)\left(\bar{\xi}-\tau \bar{\xi}_{2}\right) k_{1}^{b} \cdot k_{4}^{b}}{\bar{\xi}_{1}-\tau^{2} \bar{\xi}_{2}}, 0, \frac{(1-\tau)\left(\bar{\xi}-\tau \bar{\xi}_{2}\right) k_{1}^{b} \cdot k_{4}^{b}}{\bar{\xi}_{1}-\tau^{2} \bar{\xi}_{2}}, 0\right),
\end{aligned}
$$

$\operatorname{Res}_{\left\{\mathcal{G}_{i}\right\}} X_{2,1,1}^{* *}\left[\left(\left(\ell_{1}+k_{4}\right)^{2} / 2\right)^{2}\right]=$

$$
\begin{aligned}
& \left(0, \frac{(1-\tau)^{2}\left(\bar{\xi}-\tau \bar{\xi}_{2}\right)^{2}\left(k_{1}^{b} \cdot k_{4}^{b}\right)^{2}}{\tau\left(\bar{\xi}_{1}-\tau^{2} \bar{\xi}_{2}\right)}, 0,-\frac{(1-\tau)^{2}\left(\bar{\xi}-\tau \bar{\xi}_{2}\right)^{2}\left(k_{1}^{b} \cdot k_{4}^{b}\right)^{2}}{\tau\left(\bar{\xi}_{1}-\tau^{2} \bar{\xi}_{2}\right)}\right. \\
& \frac{(1-\tau)\left(\bar{\xi}-\tau \bar{\xi}_{2}\right)\left(k_{1}^{b} \cdot k_{4}^{b}\right)^{2}}{\tau}, 0, \frac{(1-\tau)^{2}\left(\bar{\xi}-\tau \bar{\xi}_{2}\right)^{2}\left(k_{1}^{b} \cdot k_{4}^{b}\right)^{2}}{\tau\left(\bar{\xi}_{1}-\tau^{2} \bar{\xi}_{2}\right)}, 0 \\
& \left.-\frac{(1-\tau)^{2}\left(\bar{\xi}-\tau \bar{\xi}_{2}\right)^{2}\left(k_{1}^{b} \cdot k_{4}^{b}\right)^{2}}{\tau\left(\bar{\xi}_{1}-\tau^{2} \bar{\xi}_{2}\right)}, \frac{(1-\tau)\left(\bar{\xi}-\tau \bar{\xi}_{2}\right)\left(k_{1}^{b} \cdot k_{4}^{b}\right)^{2}}{\tau}\right) \cdot
\end{aligned}
$$

The master contours that respect all integral reduction identities and extract either of the three master integrals are

$$
\begin{aligned}
& \mathcal{M}_{1}=N_{1}(1,0,-1,0,0,1,0,-1,0,0) \\
& \mathcal{M}_{2}=N_{2}\left(q_{1}, q_{2}, q_{3}, q_{4}, q_{5}, q_{1}, q_{2}, q_{3}, q_{4}, q_{5}\right) \\
& \mathcal{M}_{3}=N_{3}(1,1,1,1,2,1,1,1,1,2)
\end{aligned}
$$

In these equations, the overall constants $N_{1}, N_{2}$ and $N_{3}$ are

$$
\begin{aligned}
& N_{1} \equiv \frac{\gamma_{12} m_{1}^{2} m_{3}^{2}-\chi\left(\gamma_{12}+m_{1}^{2}\right)\left(\gamma_{12}+m_{2}^{2}\right) s_{12}}{4 m_{1}^{2}\left(m_{2}^{2}\left(\gamma_{12}+m_{1}^{2}\right)-\gamma_{12} m_{3}^{2}\right)+4 \chi \gamma_{12} s_{12}\left(\gamma_{12}+m_{1}^{2}\right)}, \\
& N_{2} \equiv \frac{1}{2\left(\gamma_{12}^{2}+m_{1}^{2} m_{2}^{2}+\gamma_{12}\left(m_{1}^{2}+m_{2}^{2}-m_{3}^{2}\right)\right)} \\
& \quad \quad \quad \frac{1}{m_{1}^{2}\left(m_{2}^{2}\left(\gamma_{12}+m_{1}^{2}\right)-\gamma_{12} m_{3}^{2}\right)+\chi \gamma_{12}\left(\gamma_{12}+m_{1}^{2}\right) s_{12}}, \\
& N_{3} \equiv \frac{\gamma_{12}\left(\gamma_{12}^{2}-m_{1}^{2} m_{2}^{2}\right)}{\gamma_{12}^{2}+m_{1}^{2} m_{2}^{2}+\gamma_{12}\left(m_{1}^{2}+m_{2}^{2}-m_{3}^{2}\right)} \\
& \quad \times \frac{1}{m_{1}^{4} m_{2}^{2}+\chi \gamma_{12}^{2} s_{12}+\gamma_{12} m_{1}^{2}\left(m_{2}^{2}-m_{3}^{2}+\chi s_{12}\right)},
\end{aligned}
$$

along with the residue weights $q_{1}, \ldots, q_{5}$,

$$
\begin{aligned}
q_{1} \equiv & \gamma_{12}^{4}-m_{1}^{4} m_{2}^{4}+\gamma_{12}^{3}\left(m_{1}^{2}+m_{2}^{2}-m_{3}^{2}-\chi s_{12}\right) \\
& -\gamma_{12} m_{1}^{2} m_{2}^{2}\left(m_{1}^{2}+m_{2}^{2}-m_{3}^{2}+\chi s_{12}\right)+\gamma_{12}^{2}\left(m_{1}^{2} m_{3}^{2}-\chi\left(m_{1}^{2}+m_{2}^{2}\right) s_{12}\right),
\end{aligned}
$$




$$
\begin{aligned}
q_{2} \equiv & m_{1}^{2}\left(-2\left(\gamma_{12}+m_{1}^{2}\right) m_{2}^{2}\left(\gamma_{12}+m_{2}^{2}\right)\right. \\
& \left.+\gamma_{12}\left(\gamma_{12}+2 m_{2}^{2}\right) m_{3}^{2}\right)-\chi \gamma_{12}\left(\gamma_{12}+m_{1}^{2}\right)\left(\gamma_{12}+m_{2}^{2}\right) s_{12}, \\
q_{3} \equiv & \gamma_{12}^{4}-m_{1}^{4} m_{2}^{4}+\gamma_{12}^{3}\left(m_{1}^{2}+m_{2}^{2}-m_{3}^{2}+\chi s_{12}\right) \\
& \quad-\gamma_{12} m_{1}^{2} m_{2}^{2}\left(m_{1}^{2}+m_{2}^{2}-m_{3}^{2}-\chi s_{12}\right)-\gamma_{12}^{2}\left(m_{1}^{2} m_{3}^{2}-\chi\left(m_{1}^{2}+m_{2}^{2}\right) s_{12}\right), \\
q_{4} \equiv & q_{1}-q_{2}+q_{3}, \\
q_{5} \equiv & q_{1}+q_{3} .
\end{aligned}
$$

We note that the three-mass projectors written here reduce to the two-mass short-side formula (5.66) in the limit $m_{3} \rightarrow 0$.

\subsection{Four-mass projectors}

We finally examine the principal kinematic configuration with four distinct external masses $m_{i}^{2}=k_{i}^{2} \neq 0$. The intermediate calculations are more complicated because neither $\gamma_{12}$ nor $\gamma_{14}$ can be simplified to rational expressions. In order to simplify the computation to the maximum extent possible prior to solving for the projectors, we will encircle a slightly different set of global poles compared to the previous examples. More specifically, we exploit that integrand of the scalar master integral, evaluated on branches three through six, has vanishing residues at infinity.

It is convenient to arrange the poles so that $\mathcal{G}_{i}$ and $\mathcal{G}_{i+5}$ are still parity conjugates of each other. The set of global poles is

$$
\left\{\mathcal{G}_{i}\right\}=\left(\mathcal{G}_{1 \cap 4}, \mathcal{G}_{1 \cap 5}, \mathcal{G}_{3, \infty_{R}}, \mathcal{G}_{5, \infty_{R}}, \mathcal{G}_{1, \infty_{L R}}, \mathcal{G}_{2 \cap 3}, \mathcal{G}_{2 \cap 6}, \mathcal{G}_{4, \infty_{R}}, \mathcal{G}_{6, \infty_{R}}, \mathcal{G}_{2, \infty_{L R}}\right)
$$

The residues at these poles can be streamlined by rescaling all hepta-cut Jacobians $J_{1}, \ldots, J_{6}$ by a common factor,

$$
J_{i} \rightarrow(1+\mu \tau)\left(\bar{\xi}_{1}-\bar{\xi}_{2}\right) J_{i},
$$

and this is what we will do implicitly below. This constant will eventually drop out when we compute integral coefficients.

Without repeating the exercise, we know that the contour constraints from parity-odd numerator insertions are

$$
\left(\begin{array}{rrrrrrrrrr}
1 & 0 & 0 & 0 & 0 & -1 & 0 & 0 & 0 & 0 \\
0 & 1 & 0 & 0 & 0 & 0 & -1 & 0 & 0 & 0 \\
0 & 0 & 1 & 0 & 0 & 0 & 0 & -1 & 0 & 0 \\
0 & 0 & 0 & 1 & 0 & 0 & 0 & 0 & -1 & 0 \\
0 & 0 & 0 & 0 & 1 & 0 & 0 & 0 & 0 & -1
\end{array}\right)\left(\begin{array}{c}
\omega_{1 \cap 4} \\
\omega_{1 \cap 5} \\
\omega_{3, \infty_{R}} \\
\omega_{5, \infty_{R}} \\
\omega_{1, \infty_{L R}} \\
\omega_{2 \cap 3} \\
\omega_{2 \cap 6} \\
\omega_{4, \infty_{R}} \\
\omega_{6, \infty_{R}} \\
\omega_{2, \infty_{L R}}
\end{array}\right)=0
$$


Prior to presenting the residues computed by the three master integrals, it proves advantageous to define the following four constants constructed out of various previously defined quantities,

$$
\begin{aligned}
& r_{1} \equiv-\frac{\tau\left(\bar{\xi}_{1}-\bar{\xi}_{2}\right)}{\bar{\xi}_{1}-\tau^{2} \bar{\xi}_{2}}, \\
& r_{2} \equiv-\frac{m_{4}^{2}}{2}-\mu(1-\tau)\left(\bar{\xi}_{1}-\tau \bar{\xi}_{2}\right) k_{1}^{b} \cdot k_{4}^{b}, \\
& r_{3} \equiv+\frac{m_{4}^{2}}{2}+(1-1 / \tau)\left(\bar{\xi}_{1}-\tau \bar{\xi}_{2}\right) k_{1}^{b} \cdot k_{4}^{b}, \\
& r_{4} \equiv-(1-1 / \tau)(1+\mu \tau)^{2}\left(\bar{\xi}_{1}-\bar{\xi}_{2}\right)\left(\bar{\xi}_{1}-\tau \bar{\xi}_{2}\right)\left(k_{1}^{b} \cdot k_{4}^{b}\right)^{2} .
\end{aligned}
$$

In terms of the $r_{i} \mathrm{~s}$, the residues of the master integrals can be brought to a particularly simple form,

$$
\begin{aligned}
\operatorname{Res}_{\left\{\mathcal{G}_{i}\right\}} X_{2,1,1}^{* *}[1] & =\left(-1, r_{1}, 0,0,0,-1, r_{1}, 0,0,0\right), \\
\operatorname{Res}_{\left\{\mathcal{G}_{i}\right\}} X_{2,1,1}^{* *}\left[\left(\ell_{1}+k_{4}\right)^{2} / 2\right] & =\left(r_{2}, r_{1} r_{3}, 0,0,0, r_{2}, r_{1} r_{3}, 0,0,0\right), \\
\operatorname{Res}_{\left\{\mathcal{G}_{i}\right\}} X_{2,1,1}^{* *}\left[\left(\left(\ell_{1}+k_{4}\right)^{2} / 2\right)^{2}\right] & =\left(-r_{2}^{2}, r_{1} r_{3}^{2}, 0,0, r_{4},-r_{2}^{2}, r_{1} r_{3}^{2}, 0,0, r_{4}\right) .
\end{aligned}
$$

There are again two linearly independent consistency equations arising from the IBP identities,

$$
\begin{aligned}
& \boldsymbol{\Omega} \cdot(1,0,2,0,-1,1,0,2,0,-1)=0, \\
& \boldsymbol{\Omega} \cdot(0,1,0,2,-1,0,1,0,2,-1)=0 .
\end{aligned}
$$

At first sight these constraints differ from those found in the two- and three-mass calculations, see e.g. eqs. (5.64)-(5.65). However, the two pairs of equations enforce the same constraints, as can be argued easily. We may express the constraints without imposing the global residue theorem. For the four-mass case we then have

$$
\begin{gathered}
\omega_{1 \cap 3}+\omega_{1 \cap 4}+\omega_{2 \cap 3}+\omega_{2 \cap 4} \\
-\omega_{1, \infty_{L R}}-\omega_{2, \infty_{L R}}-\omega_{1, \infty}-\omega_{2, \infty}+2 \omega_{3, \infty}+2 \omega_{4, \infty}=0, \\
\omega_{1 \cap 5}+\omega_{1 \cap 6}+\omega_{2 \cap 5}+\omega_{2 \cap 6} \\
-\omega_{1, \infty_{L R}}-\omega_{2, \infty_{L R}}-\omega_{1, \infty}-\omega_{2, \infty}+2 \omega_{5, \infty}+2 \omega_{6, \infty}=0 .
\end{gathered}
$$

These equations encompass either form of the IBP constraints, i.e. eqs. (5.64)-(5.65) and (5.103)-(5.104). This can be shown by writing out the latter explicitly, i.e.

$$
\begin{aligned}
& \omega_{1 \cap 3}+\omega_{1 \cap 4}+\omega_{2 \cap 3}+\omega_{2 \cap 4}-\omega_{1, \infty_{L R}}-\omega_{2, \infty_{L R}}=0, \\
& \omega_{1 \cap 5}+\omega_{1 \cap 6}+\omega_{2 \cap 5}+\omega_{2 \cap 6}-\omega_{1, \infty_{L R}}-\omega_{2, \infty_{L R}}=0,
\end{aligned}
$$

and

$$
\begin{aligned}
& \omega_{1 \cap 4}+\omega_{2 \cap 3}-\omega_{1, \infty_{L R}}-\omega_{2, \infty_{L R}}-\omega_{1, \infty}-\omega_{2, \infty}+2 \omega_{3, \infty}+2 \omega_{4, \infty}=0, \\
& \omega_{1 \cap 5}+\omega_{2 \cap 6}-\omega_{1, \infty_{L R}}-\omega_{2, \infty_{L R}}-\omega_{1, \infty}-\omega_{2, \infty}+2 \omega_{5, \infty}+2 \omega_{6, \infty}=0 .
\end{aligned}
$$


Then it is immediately clear that we obtain the same answer from eqs. (5.105)-(5.106) when we truncate to linearly independent sets of residues by the global residue theorem.

Returning to actual calculation with the representation (5.102) of the residues at hand, it is quite easy to solve the set of linear equations to derive compact expressions for the three master integral projectors,

$$
\begin{gathered}
\mathcal{M}_{1}=-\frac{1}{4 r_{1}\left(r_{2}+r_{3}\right) r_{4}}\left(2 r_{1} r_{3} r_{4},-2 r_{2} r_{4}, r_{1} r_{3}\left(r_{2}^{2}+r_{2} r_{3}-r_{4}\right),\right. \\
r_{2}\left(r_{1} r_{3}\left(r_{2}+r_{3}\right)+r_{4}\right), 2 r_{1} r_{2} r_{3}\left(r_{2}+r_{3}\right), \\
2 r_{1} r_{3} r_{4},-2 r_{2} r_{4}, r_{1} r_{3}\left(r_{2}^{2}+r_{2} r_{3}-r_{4}\right), \\
\left.r_{2}\left(r_{1} r_{3}\left(r_{2}+r_{3}\right)+r_{4}\right), 2 r_{1} r_{2} r_{3}\left(r_{2}+r_{3}\right)\right) \\
\mathcal{M}_{2}=+\frac{1}{4 r_{1}\left(r_{2}+r_{3}\right) r_{4}}\left(2 r_{1} r_{4}, 2 r_{4}, r_{1}\left(r_{2}^{2}-r_{3}^{2}-r_{4}\right), r_{1}\left(r_{2}^{2}-r_{3}^{2}\right)-r_{4}, 2 r_{1}\left(r_{2}^{2}-r_{3}^{2}\right),\right. \\
\left.2 r_{1} r_{4}, 2 r_{4}, r_{1}\left(r_{2}^{2}-r_{3}^{2}-r_{4}\right), r_{1}\left(r_{2}^{2}-r_{3}^{2}\right)-r_{4}, 2 r_{1}\left(r_{2}^{2}-r_{3}^{2}\right)\right), \\
\mathcal{M}_{3}=\frac{1}{4 r_{4}}(0,0,1,1,2,0,0,1,1,2) .
\end{gathered}
$$

This result completes our derivation of master integral projectors for the two-loop crossed box with up to four massive external legs.

\section{Reduction of integrals with doubled propagators}

Feynman integrals with doubled and in general higher powers of propagators frequently appear in loop amplitude computations, for instance in IBP identities, Schwinger parametrizations or bubble insertions. It was recently explained that generalized unitarity cuts of such integrals are naturally treated as degenerate multivariate residues using computational algebraic geometry [52]. In that connection, several examples were given for one- and two-loop integrals with massless kinematics. This method extends seamlessly to multiloop integrals with external masses, as we will demonstrate shortly. However, the calculation can be accelerated by using the Bezoutian matrix algorithm.

\subsection{Unitarity cut algorithm: Bezoutian matrix method}

We very briefly review the unitarity cut algorithm for integrals with higher powers of propagators. For more details and examples, please refer to refs. [51, 52]. The main ingredient needed is computational algebraic geometry.

Recall that a residue is nondegenerate, if the Jacobian at the pole $\xi$ is nonzero, i.e.,

$$
J(\xi)=\left.\operatorname{det}_{i, j}\left(\frac{\partial f_{i}}{\partial z_{j}}\right)\right|_{z=\xi} \neq 0 .
$$

In this case, the value of the residue is simply calculated by Cauchy's theorem in higher dimensions, i.e. eq. (2.9). However, the Jacobian clearly vanishes if there is one or more 
doubled propagators being cut and the residue is degenerate, so this approach does not apply. To solve the problem, we need techniques from algebraic geometry. There are two ways of evaluating such residues:

1. The transformation law (see for instance ref. [72]). This theorem can be used to convert a degenerate residue at the simultaneous zero of the inverse propagators to a factorizable residue. The explicit transformation matrix is found by the Gröbner bases method. The algorithm is described in refs. [51, 52].

2. The Bezoutian matrix method. Here one determines the duality structure [71] of the multivariate residues, which in turn can be calculated easily. In general, the Bezoutian matrix method is considerably faster than the transformation law for complicated cuts with many independent external invariants.

Our Mathematica package MathematicaM2 ${ }^{2}$ is capable of computing multivariate residues using either of these techniques. In what follows, we outline the Bezoutian matrix approach and provide some basic examples.

Let $I=\left\langle f_{1}, \ldots, f_{n}\right\rangle$ be an ideal in the ring $R=\mathbb{C}\left[z_{1}, \ldots, z_{n}\right]$. Assume that $I$ is a zero-dimensional ideal, i.e. the zero locus $\mathcal{Z}(I)=\left\{\xi_{1}, \ldots, \xi_{k}\right\}$ consists of finite number of discrete points. For a zero-dimensional ideal $I$, the quotient ring $R / I$ is a finite dimensional C-linear space.

Before we calculate individual residues, we first examine the structure of the sum of residues by Bezoutian Matrix. Then we eventually get individual residues from partition functions. For a polynomial $h$ in $R$, we define the global residue as

$$
\operatorname{Res}(h)=\sum_{i} \operatorname{Res}_{\xi_{i}}\left(\frac{h d z_{1} \wedge \cdots \wedge d z_{n}}{f_{1} \cdots f_{n}}\right)
$$

which is just the sum of all residues. By Stokes' theorem, the values of the residues only depend on $h$ 's equivalence class $[h]$ in $R / I$. Furthermore, we can define an inner product $\langle$,$\rangle in R / I$,

$$
\langle g, h\rangle \equiv \operatorname{Res}(g \cdot h) .
$$

Theorem $1\langle$,$\rangle is a nondegenerate inner product in R / I$.

The proof of the theorem is given in ref. [72]. This theorem implies that, given a linear basis $\left\{p_{i}\right\}$ for $R / I$, we can find its dual basis $\left\{\Delta_{i}\right\}$ in $R / I$, such that

$$
\left\langle p_{i}, \Delta_{j}\right\rangle=\delta_{i j}
$$

In practice, the basis and dual basis can be found by the Gröbner basis method and the Bezoutian matrix [74]. The procedure involves the following steps:

1. Calculate $G$, the Gröbner basis of $I$ in the DegreeLexicographic order. Denote the leading terms for all polynomials in $G$ as $L T(G)$. Then all monomials in $R$ which are lower than $L T(G)$ constitute $\left\{p_{i}\right\}$, which is the canonical linear basis for $R / I$.

\footnotetext{
${ }^{2}$ The package can be downloaded from https://bitbucket.org/yzhphy/mathematicam2.
} 
2. Introduce a set of auxiliary variables $\left\{y_{1}, \ldots, y_{n}\right\}$ and define the Bezoutian matrix $B$ for $I$ as,

$$
B_{i j} \equiv \frac{f_{i}\left(y_{1}, \ldots, y_{j-1}, z_{j}, \ldots, z_{n}\right)-f_{i}\left(y_{1}, \ldots, y_{j}, z_{j+1}, \ldots, z_{n}\right)}{z_{j}-y_{j}}
$$

Calculate its determinant, $\operatorname{det} B$.

3. Define $\tilde{G}$ as the set $G$ after the replacement $z_{i} \rightarrow y_{i}$. Perform the polynomial division of the Bezoutian determinant, $\operatorname{det} B$, over $G \otimes \tilde{G}$. Then the remainder can be written,

$$
\sum_{i} a_{i}(y) p_{i}(z)
$$

where the $p_{i}$ s form the canonical linear basis for $R / I$, and the $a_{i}(y) \mathrm{s}$ are polynomials in the $y$-variables only.

4. The dual basis $\left\{\Delta_{i}\right\}$, with respect to the inner product $\langle$,$\rangle , is defined as \Delta_{i}=a_{i}(z)$.

The dual basis explicitly characterizes the structure of global residues. Let the decomposition of the unit 1 over the dual basis be given as

$$
1=\sum_{i} \mu_{i} \Delta_{i}
$$

Then for an arbitrary numerator $h$, expand $[h]$ over the canonical linear basis,

$$
[h]=\sum_{i} \lambda_{i} p_{i}
$$

and the global residue is given as [74],

$$
\operatorname{Res}(h)=\sum_{i} \lambda_{i} \mu_{i}
$$

This formula is the result of the definition of the dual basis, and provides a very efficient way of calculating the residues.

To get individual residues, we can use the formula (6.9) and the new ingredient partition functions of $\mathcal{Z}(I)$.

Theorem 2 Let $I$ be a zero-dimensional ideal and $\mathcal{Z}(I)=\left\{\xi_{1}, \ldots, \xi_{k}\right\}$ be its zero locus. Denote $\mathcal{O}_{i}$ as the local ring of $\xi, \mathcal{O}_{i}=\left\{f / g \mid g\left(\xi_{i}\right) \neq 0, f \in R, g \in R\right\}$, and $I \mathcal{O}_{i}$ as the ideal generated by $I$ in $O_{i}$. Then there is a set of partition functions, $e_{1}, \ldots, e_{k}$, each of which is an element in $R / I$, such that,

1. In $R / I, \sum_{i} e_{i}=1, e_{i}^{2}=e_{i}$ and $e_{i} e_{j}=0$ if $i \neq j$.

2. $e_{i} \in I \mathcal{O}_{j}$ if $i \neq j$, and $e_{i}-1 \in I \mathcal{O}_{i}$. 
This theorem can be proved by construction [75]. Then for each individual residue at $\xi_{i}[74]$, we have the result

$$
\operatorname{Res}_{\xi_{i}}\left(\frac{h d z_{1} \wedge \cdots \wedge d z_{n}}{f_{1} \cdots f_{n}}\right)=\operatorname{Res}\left(h \cdot e_{i}\right) .
$$

Explicitly, the partition functions $e_{1}, \ldots, e_{k}$ can be constructed by the method of Lagrange interpolation. The computation via Bezoutian matrix method is realized in our package, MathematicaM2. We demonstrate this computation by a simple example before we return to generalized unitarity cuts of integrals with doubled propagators. To compare with the transform law method described in [50,51], we present some one-loop and two-loop residues computations in Example 2, 3.

Example 1 Let $I=\left\langle\left(z_{1}+z_{2}\right)^{2}, z_{2}^{2}+z_{1}^{2}-2\right\rangle$. There are two residues located at $\xi_{1}=(-1,1)$ and $\xi_{2}=(1,-1)$. Both residues are degenerate. From the Gröbner basis computation, the linear basis $\left\{p_{i}\right\}$ for $R / I$ is,

$$
\left\{z_{2}^{2}, z_{1}, z_{2}, 1\right\}
$$

The Bezoutian matrix is,

$$
B=\left(\begin{array}{cc}
y_{1}+z_{1}+2 z_{2} & 2 y_{1}+y_{2}+z_{2} \\
y_{1}+z_{1} & y_{2}+z_{2}
\end{array}\right) .
$$

Its determinant is $\operatorname{det} B=-2 y_{1} z_{1}+2 y_{2} z_{2}-2 y_{1}^{2}+2 z_{2}^{2}$. So after the polynomial division over the Gröbner basis of $I$, we have the dual basis,

$$
\left\{\Delta_{i}\right\}=\left\{2,-2 z_{1}, 2 z_{2}, 2\left(z_{2}^{2}-2\right)\right\} .
$$

Consider the numerator $h=z_{2}^{2}$. From the dual basis structure, we immediately get decomposition,

$$
1=\frac{1}{2} \Delta_{1}
$$

Hence $\left\{\mu_{i}\right\}=\left\{\frac{1}{2}, 0,0,0\right\}$. Now it is clear that,

$$
\operatorname{Res}(h)=\frac{1}{2}
$$

Furthermore, we construct partition functions for this ideal according to ref. [75]. By Lagrangian interpolation, we obtain two polynomials

$$
l_{1}=-\frac{1}{2}\left(z_{1}-1\right), \quad l_{2}=\frac{1}{2}\left(z_{1}+1\right),
$$

such that $l_{i}\left(\xi_{j}\right)=\delta_{i j}, i, j=1,2$. Since the two poles both have multiplicity 2 , the partition functions are [75],

$$
\begin{aligned}
& e_{1}=1-\left(1-l_{1}^{2}\right)^{2}=\frac{1}{4}\left(2-2 z_{1}+z_{2}\right) \quad \bmod I \\
& e_{2}=1-\left(1-l_{2}^{2}\right)^{2}=\frac{1}{4}\left(2+2 z_{1}-z_{2}\right) \quad \bmod I
\end{aligned}
$$


Here, to simplify the expression, we performed a polynomial division over I. Then the local residues are,

$$
\begin{aligned}
& \operatorname{Res}_{\xi_{1}}\left(\frac{h d z_{1} \wedge \cdots \wedge d z_{n}}{f_{1} \cdots f_{n}}\right)=\operatorname{Res}\left(h \cdot e_{1}\right)=\frac{1}{4}, \\
& \operatorname{Res}_{\xi_{2}}\left(\frac{h d z_{1} \wedge \cdots \wedge d z_{n}}{f_{1} \cdots f_{n}}\right)=\operatorname{Res}\left(h \cdot e_{2}\right)=\frac{1}{4} .
\end{aligned}
$$

Example 2 We calculate the two multivariate residues from the maximal cut of the oneloop massless box diagram and then compare the efficiency of Bezoutian method and the transformation law method. The Feynman integral of the box diagram is,

$$
I_{\square}\left(\sigma_{1}, \ldots, \sigma_{4}\right) \equiv \int_{\mathbb{R}^{D}} \frac{d^{D} \ell}{(2 \pi)^{D}} \prod_{k=1}^{4} \frac{1}{f_{k}^{\sigma_{k}}(\ell)},
$$

where the denominators are,

$$
f_{1}=\ell^{2}, \quad f_{2}=\left(\ell-k_{1}\right)^{2}, \quad f_{3}=\left(\ell-k_{1}-k_{2}\right)^{2}, \quad f_{4}=\left(\ell+p_{4}\right)^{2} .
$$

We parametrize the loop momentum $\ell$ as,

$$
\ell^{\mu}=\alpha_{1} k_{1}^{\mu}+\alpha_{2} k_{2}^{\mu}+\frac{\alpha_{3} s_{12}}{2\langle 14\rangle[42]}\left\langle 1\left|\gamma^{\mu}\right| 2\right\rangle+\frac{\alpha_{4} s_{12}}{2\langle 24\rangle[42]}\left\langle 2\left|\gamma^{\mu}\right| 1\right\rangle .
$$

The box integrand has two quadruple-cut poles,

$$
\left(\alpha_{1}, \alpha_{2}, \alpha_{3}, \alpha_{4}\right)=(1,0,0,-\chi) \equiv \xi_{1}, \quad\left(\alpha_{1}, \alpha_{2}, \alpha_{3}, \alpha_{4}\right)=(1,0,-\chi, 0) \equiv \xi_{2} .
$$

Consider the residue of the triple propagator integral $I_{\square}(3,1,1,1)$. The dual basis $\left\{\Delta_{i}\right\}$ from the Bezoutian matrix computation is

$$
\left\{\Delta_{i}\right\}=\frac{s_{12}^{6}}{\chi^{3}(1+\chi)}\left\{-1, \alpha_{3},-\alpha_{4}-3 \chi, \alpha_{3}\left(\alpha_{3}+3 \chi\right),-\alpha_{4}^{2}-3 \alpha_{4} \chi-3 \chi^{2},-\left(\alpha_{4}+\chi\right)^{3}\right\},
$$

and the partition functions are,

$$
\left\{e_{1}, e_{2}\right\}=\frac{1}{\chi^{3}}\left\{-\alpha_{4}\left(\alpha_{4}^{2}+3 \alpha_{4} \chi+3 \chi^{2}\right),\left(\alpha_{4}+\chi\right)^{3}\right\} .
$$

Then we have

$$
\begin{aligned}
& \operatorname{Res}_{\xi_{1}}\left(\frac{d \alpha_{1} \wedge \cdots \wedge d \alpha_{4}}{f_{1}^{3} f_{2} f_{3} f_{4}}\right)=\operatorname{Res}\left(e_{1}\right)=+\frac{1+\chi}{s_{12}^{6}}, \\
& \operatorname{Res}_{\xi_{2}}\left(\frac{d \alpha_{1} \wedge \cdots \wedge d \alpha_{4}}{f_{1}^{3} f_{2} f_{3} f_{4}}\right)=\operatorname{Res}\left(e_{2}\right)=-\frac{1+\chi}{s_{12}^{6}} .
\end{aligned}
$$

This is a very simple example. Using the package MathematicaM2, the whole computation takes 0.54 seconds, via Bezoutian method. The computation based on transformation law [52], gives the same result, but takes 1.07 seconds. There is no significant efficiency difference between the two methods, for this one-loop example. 
Example 3 We calculate one of the multivariate residues from the maximal cut of the twoloop massless double-box diagram and again compare the efficiency of Bezoutian method and the transformation law method. The Feynman integral of the double-box diagram is,

$$
P_{2,2}^{* *}\left(\sigma_{1}, \ldots, \sigma_{7}\right) \equiv \int_{\mathbb{R}^{D}} \frac{d^{D} \ell_{1}}{(2 \pi)^{D}} \int_{\mathbb{R}^{D}} \frac{d^{D} \ell_{2}}{(2 \pi)^{D}} \prod_{k=1}^{7} \frac{1}{f_{k}^{\sigma_{k}}(\ell)},
$$

where the denominators are,

$$
\begin{array}{lll}
f_{1}=\ell_{1}^{2}, & f_{2}=\left(\ell_{1}-k_{1}\right)^{2}, & f_{3}=\left(\ell_{1}-k_{1}-k_{2}\right)^{2}, \\
f_{4}=\ell_{2}^{2}, & f_{5}=\left(\ell_{2}-k_{4}\right)^{2}, & f_{6}=\left(\ell_{2}-k_{3}-k_{4}\right)^{2},
\end{array}
$$

The loop momenta are parametrized as,

$$
\begin{aligned}
& \ell_{1}^{\mu}=\alpha_{1} k_{1}^{\mu}+\alpha_{2} k_{2}^{\mu}+\frac{\alpha_{3} s_{12}}{\langle 14\rangle[42]}\left\langle 1\left|\gamma^{\mu}\right| 2\right\rangle+\frac{\alpha_{4} s_{12}}{\langle 24\rangle[41]}\left\langle 2\left|\gamma^{\mu}\right| 1\right\rangle, \\
& \ell_{2}^{\mu}=\beta_{1} k_{3}^{\mu}+\beta_{2} k_{4}^{\mu}+\beta_{3}+\frac{\beta_{3} s_{12}}{\langle 31\rangle[14]}\left\langle 3\left|\gamma^{\mu}\right| 4\right\rangle+\frac{\beta_{4} s_{12}}{\langle 41\rangle[13]}\left\langle 4\left|\gamma^{\mu}\right| 3\right\rangle .
\end{aligned}
$$

Consider the residue at

$$
\left(a_{1}, a_{2}, a_{3}, a_{4}, b_{1}, b_{2}, b_{3}, b_{4}\right)=(1,0,-\chi, 0,0,1, z, 0) \equiv \xi .
$$

By the Bezoutian matrix method, we find that

$$
\operatorname{Res}_{\xi}\left(\frac{d \alpha_{1} \wedge \cdots \wedge d \alpha_{4} \wedge d \beta_{1} \wedge \cdots \wedge d \beta_{4}}{f_{1} f_{2} f_{3} f_{4} f_{5} f_{6} f_{7}^{3}}\right)=\frac{\chi^{2}(1+\chi)^{2}}{z(z+\chi)^{3}}
$$

The computation takes 1.28 seconds with a numerical value of $\chi$. The transformation law methods takes 10.7 seconds with a numerical value of $\chi$. So for this two-loop example, the Bezoutian method is about 8 times faster.

\subsection{Example: one-mass two-loop crossed box}

The previous examples show that the Bezoutian matrix algorithm is significantly faster than the transformation law method for massless two-loop problems. The difference is even more profound for nonplanar diagrams with external masses, as we shall see shortly.

We will be slightly more general than in eq. (3.2) and define the two-loop crossed box integral with arbitrary integer powers $\left(\sigma_{1}, \ldots, \sigma_{9}\right)$ of propagators and irreducible numerators as

$$
X_{2,1,1}^{* *}\left(\sigma_{1}, \ldots, \sigma_{9}\right) \equiv \int_{\mathbb{R}^{D}} \frac{d^{D} \ell_{1}}{(2 \pi)^{D}} \int_{\mathbb{R}^{D}} \frac{d^{D} \ell_{2}}{(2 \pi)^{D}} \prod_{k=1}^{9} \frac{1}{f_{k}^{\sigma k}\left(\ell_{1}, \ell_{2}\right)}
$$

where the seven propagators $f_{1}, \ldots, f_{7}$ can be found in eq. (3.1) with $k_{5}=k_{6}=0$ and

$$
f_{8} \equiv \frac{1}{2}\left(\ell_{1}+k_{4}\right)^{2}, \quad f_{9} \equiv \frac{1}{2}\left(\ell_{2}+k_{1}\right)^{2} .
$$




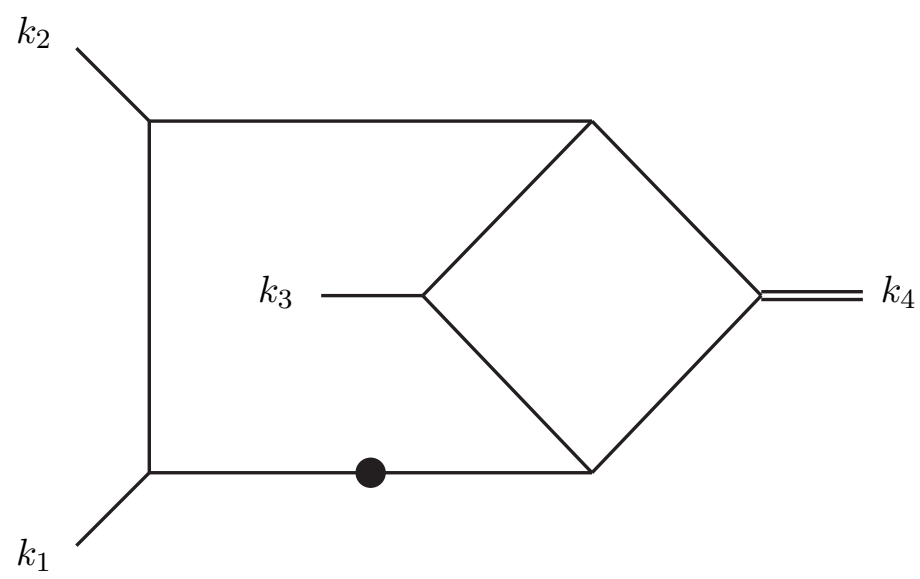

Figure 7. The four-point two-loop crossed-box diagram with one external massive leg and a doubled propagator, which is depicted by a black dot. The massive momentum $k_{4}$ is marked by a doubled line.

In order to unambiguously define the degenerate multivariate residue associated with the maximal cut, the inverse propagators are grouped into seven factors $g_{i} \equiv f_{i}^{\sigma_{i}}$. As in [52] we will for technical simplicity only consider cuts in strictly four dimensions, postponing the analysis in $D=4-2 \epsilon$ dimensions to future work.

For simplicity we assume for now that $m_{1} m_{2}=0$ so that a general integral can be expanded onto a basis of two masters,

$$
X_{2,1,1}^{* *}\left(\sigma_{1}, \ldots, \sigma_{9}\right)=c_{1} X_{2,1,1}^{* *}(1, \ldots, 1,0,0)+c_{2} X_{2,1,1}^{* *}(1, \ldots, 1,-1,0)+\cdots .
$$

As an example, we will examine the one-mass doubled propagator integral with indices $(2,1, \ldots, 1,0,0)$ (see figure 7 ) and reconstruct the coefficients $c_{1}$ and $c_{2}$ to leading order in dimensional regularization using the projectors derived in the previous section. Let $m_{1}=m_{2}=m_{3}=0$ and $m_{4} \neq 0$. The degenerate multivariate residues at the simultaneous zeros of the inverse propagators specified by $\mathcal{S}_{1}^{\prime}, \ldots, \mathcal{S}_{8}^{\prime}$ are computed using our package, MathematicaM2,

$$
\begin{aligned}
& X_{2,1,1}^{* *}(2,1, \ldots, 1,0,0)_{\widetilde{\mathcal{S}}_{1,2}}=+\frac{\gamma_{\star}}{\chi^{2} s_{12}^{2}} \oint d z \frac{s_{12}(2(1+\chi)+(1+2 \chi) z)-m_{4}^{2}}{z(z+1)^{2}\left(z+\left(s_{12}(1+\chi)-m_{4}^{2}\right) /\left(\chi s_{12}\right)\right)^{2}}, \\
& X_{2,1,1}^{* *}(2,1, \ldots, 1,0,0)_{\widetilde{\mathcal{S}}_{3,4}}=-\frac{\gamma_{\star}}{\chi^{2} s_{12}^{2}} \oint d z \frac{s_{12}(2(1+\chi)+(1+2 \chi) z)-m_{4}^{2}}{z(z+1)^{2}\left(z+\left(s_{12}(1+\chi)-m_{4}^{2}\right) /\left(\chi s_{12}\right)\right)^{2}} \\
& X_{2,1,1}^{* *}(2,1, \ldots, 1,0,0)_{\widetilde{\mathcal{S}}_{5,6}}=-\frac{\gamma_{\star}}{s_{12}} \oint d z \frac{2+z}{(z+1)^{2}\left(z+m_{4}^{2} / s_{12}\right)} \\
& X_{2,1,1}^{* *}(2,1, \ldots, 1,0,0)_{\widetilde{\mathcal{S}}_{7,8}}=-\frac{\gamma_{\star}}{m_{4}^{2}} \oint d z \frac{h(z)}{(z+\mu)^{3}(z-1 / \tau)^{2}}
\end{aligned}
$$

In these equations, $h$ is a cubic polynomial,

$$
h(z)=\mu \tau z^{3}-\mu(2-(3+\tau) \mu \tau) z^{2}-\mu^{2}(4+\tau(1-\mu \tau)) z-\mu^{3} \tau .
$$


The variables $\mu$ and $\tau$ as functions of $\chi, s_{12}$ and $m_{4}$ are

$$
\mu=\frac{m_{4}^{2}\left(m_{4}^{2}-(1+\chi) s_{12}\right)}{\chi s_{12}^{2}}, \quad \tau=\frac{\chi s_{12}}{(1+\chi) s_{12}-m_{4}^{2}} .
$$

Actually, we do not explicitly need the doubled propagator hepta-cuts for $\widetilde{\mathcal{S}}_{5}, \ldots, \widetilde{\mathcal{S}}_{8}$ as the Jacobian poles are located on the intersections between the on-shell branches and no further poles are generated [52]. Given these hepta-cut integrals, it is easy to reproduce the residue relations.

It is convenient to strip off the overall factor $\gamma_{\star}=\left(16 \chi s_{12}^{3}\right)^{-1}$ from all hepta-cuts as we will do now. The residues computed by the doubled propagator scalar integral at the singular point locus (5.12) thus read

$$
\begin{aligned}
\operatorname{Res}_{\left\{\mathcal{G}_{i}\right\}} X_{2,1,1}^{* *}(2,1, \ldots, 1,0,0) & \\
& =\frac{1}{\left(m_{4}^{2}-s_{12}\right)^{2}\left(m_{4}^{2}-(1+\chi) s_{12}\right)^{2}}\left(r_{1}, r_{2},-r_{1},-r_{2}, r_{3}, r_{1}, r_{2},-r_{1},-r_{2}, r_{3}\right),
\end{aligned}
$$

for $r_{1}, r_{2}$ and $r_{3}$ given by

$$
\begin{aligned}
& r_{1} \equiv\left(m_{4}^{2}-2 s_{12}\right)\left(m_{4}^{2}-(1+\chi) s_{12}\right)^{2}, \\
& r_{2} \equiv \chi s_{12}^{2}\left(2(1+\chi) s_{12}-(2+\chi) m_{4}^{2}\right), \\
& r_{3} \equiv\left(m_{4}^{2}-s_{12}\right)^{2}\left(2(1+\chi) s_{12}-m_{4}^{2}\right) .
\end{aligned}
$$

This information allows us to derive the desired coefficients by applying the relevant projectors (5.30). The result is as follows,

$$
\begin{aligned}
X_{2,1,1}^{* *}(2,1, \ldots, 1,0,0)= & +\frac{m_{4}^{2}-2 s_{12}}{\left(m_{4}^{2}-s_{12}\right) s_{12}} X_{2,1,1}^{* *}(1, \ldots, 1,0,0) \\
& -\frac{2 m_{4}^{2}}{\left(m_{4}^{2}-s_{12}\right)\left(m_{4}^{2}-(1+\chi) s_{12}\right) s_{12}} X_{2,1,1}^{* *}(1, \ldots, 1,-1,0)+\cdots
\end{aligned}
$$

which is consistent with the $D=4$ limit of the following IBP relation in $D=4-2 \epsilon$ dimensions generated by FIRE [70],

$$
\begin{aligned}
& X_{2,1,1}^{* *}(2,1, \ldots, 1,0,0)= \\
& +\frac{(1+2 \epsilon)\left(2(1+\chi)(1+\epsilon) s_{12}^{2}+m_{4}^{2}\left(m_{4}^{2}(1+\epsilon)-(\chi+3(1+\epsilon)) s_{12}\right)\right.}{\left.(1+\epsilon) s_{12}\left(m_{4}^{2}-s_{12}\right)\left(m_{4}^{2}-(1+\chi) s_{12}\right)\right)} X_{2,1,1}^{* *}(1, \ldots, 1,0,0) \\
& \quad-\frac{2(1+2 \epsilon)(1+4 \epsilon) m_{4}^{2}}{(1+\epsilon)\left(m_{4}^{2}-s_{12}\right)\left(m_{4}^{2}-(1+\chi) s_{12}\right) s_{12}} X_{2,1,1}^{* *}(1, \ldots, 1,-1,0)+\cdots .
\end{aligned}
$$

It only takes a few seconds to obtain each of the degenerate multivariate residues (6.37) in Mathematica using the Bezoutian matrix algorithm and numerical values for the external invariants. Remarkably, the Bezoutian matrix algorithm is at least $20-25$ times faster compared to the transformation law method for this problem and in purely analytic mode, the difference is even more significant. 


\begin{tabular}{|c|c|c|c|c|c|c|c|c|}
\hline$m_{1}$ & $m_{2}$ & $m_{3}$ & $m_{4}$ & $|\mathcal{S}|$ & Res & Odd & Even & MIs \\
\hline$\neq 0$ & 0 & 0 & 0 & 8 & 10 & 5 & 3 & $(0,0),(1,0)$ \\
\hline 0 & 0 & 0 & $\neq 0$ & 8 & 10 & 5 & 3 & $(0,0),(1,0)$ \\
\hline$\neq 0$ & 0 & $\neq 0$ & 0 & 8 & 10 & 5 & 3 & $(0,0),(1,0)$ \\
\hline$\neq 0$ & 0 & 0 & $\neq 0$ & 8 & 10 & 5 & 3 & $(0,0),(1,0)$ \\
\hline$\neq 0$ & 0 & $\neq 0$ & $\neq 0$ & 8 & 10 & 5 & 3 & $(0,0),(1,0)$ \\
\hline$\neq 0$ & $\neq 0$ & 0 & 0 & 6 & 10 & 5 & 2 & $(0,0),(1,0),(2,0)$ \\
\hline$\neq 0$ & $\neq 0$ & $\neq 0$ & 0 & 6 & 10 & 5 & 2 & $(0,0),(1,0),(2,0)$ \\
\hline$\neq 0$ & $\neq 0$ & $\neq 0$ & $\neq 0$ & 6 & 10 & 5 & 2 & $(0,0),(1,0),(2,0)$ \\
\hline
\end{tabular}

Table 1. Classification of all kinematically distinct diagrams from the viewpoint of the maximal cut. The columns list whether the external masses $m_{1}, m_{2}, m_{3}$ and $m_{4}$ are zero or not, the number $|\mathcal{S}|$ of hepta-cut solutions, the number of independent residues, the number of parity-odd and parity-even contour constraints and finally the set of master integrals. The notation for the master integrals refers to the powers of the two irreducible numerator insertions.

\section{Discussion and conclusion}

In the present paper we have extended the four-dimensional maximal unitarity method [46, 47 to two-loop integrals with crossed box topology with one through four massive external legs. In practice, these integrals cover most of the nonplanar basis at four points [69]. In generalized unitarity, amplitudes are expanded onto a basis of master integrals with rational coefficients which are then extracted systematically by taking cuts that promote multiple internal lines simultaneously to on-shell regions of momentum space. As this in general involves complex kinematics, cuts are realized by replacing real slice integrations by multidimensional contours that encircle the global poles of the loop integrand. These contours are subject to the consistency requirement that the unitarity procedure respects the reduction onto master integrals which relies on vanishing of parity-odd integrands and total derivatives upon integration [46].

Our principal result is unique analytic contours for all basis integral coefficients in all inequivalent configurations of massive and massless external momenta in the four-point two-loop crossed box, valid to $\mathcal{O}\left(\epsilon^{0}\right)$ in the dimensional regulator. The content of this paper is also relevant for higher-multiplicity scattering of massless particles. The maximal cut defines a nodal algebraic curve associated with a hextuply or octuply pinched genus-3 Riemann surface whose components are Riemann spheres. The first category includes contributions where both legs in the planar end of the diagram are massive, whereas the second covers the rest. The number of linearly independent residues is always ten as expected. We find that for the sixfold degenerate curve, the projectors for all three master integrals are unique once we impose five linearly independent Levi-Civita constraints and two linearly independent IBP conditions. The Levi-Civita constraints are resolved for weights that respect parity. In the four-mass case, unlike the situation for the four-mass double box [49], the IBP constraints are not satisfied automatically for the two-loop crossed box.

Overall, the results exhibit a very interesting and naively unexpected simplicity, which clearly deserves more attention. Indeed, the systematics of the contour constraints (e.g. 
eqs. (5.64)-(5.65)) are remarkable. Instead of being a set of disconnected calculations, the inequivalent kinematic configurations related through a rich underlying structure that seems to be governed by the global picture of the hepta-cut. As summarized in table 1, we find that the contour constraints are identical for all configurations within a particular class of hepta-cut solutions, for example all the way from the three-mass diagram with $m_{1} m_{2}=0$ to the purely massless case. The IBP contours seem to be even more systematic. In all cases with six hepta-cut solutions we find two linearly independent IBP constraints. The chiral branching from $6 \rightarrow 8$ hepta-cut solutions triggers the emergence of an additional IBP constraint. Interestingly, as for the planar double box [49], the IBP constraints are inherited through chiral branchings. To see this, let us instead consider hepta-cuts from the eightfold degenerate genus- 3 curve, with the set of global poles,

$$
\left(\widetilde{\mathcal{G}}_{1 \cap 6}, \widetilde{\mathcal{G}}_{1 \cap 8}, \widetilde{\mathcal{G}}_{3 \cap 5}, \widetilde{\mathcal{G}}_{3 \cap 7}, \widetilde{\mathcal{G}}_{3, \infty_{R}}, \widetilde{\mathcal{G}}_{2 \cap 5}, \widetilde{\mathcal{G}}_{2 \cap 7}, \widetilde{\mathcal{G}}_{4 \cap 6}, \widetilde{\mathcal{G}}_{4 \cap 8}, \widetilde{\mathcal{G}}_{4, \infty_{R}}\right) \text {. }
$$

As pointed out in appendix B, these poles exactly correspond to poles on the hextuply pinched genus-3 curve. It is now an easy task to check that two of the three IBP constraints are inherited. The same observation applies to the four-mass computation.

In view of the complexity of the hepta-cut expressions and the typical amount of effort required to generate IBP relations for high-rank integrals with many external relations, it is also striking that the constraints coefficients are simply integers. This also applies to the planar double box with up to four external masses [46, 48, 49] and the planar triple box [50]. This is a clear hint of a general principle that may be explained by algebraic geometry.

The last part of this paper described a new algorithm based on the Bezoutian matrix and Gröbner bases to compute degenerate multivariate residues which typically appear in more complicated calculations. This algorithm was applied to a few simple examples and to the reduction of a massive two-loop crossed box integral with a doubled propagator onto master integrals with only single propagators. Our tests have shown that the Bezoutian matrix algorithm is considerably faster than the transformation law method [51] for twoloop problems. The multivariate residues from the massive two-loop crossed box integral are computed at least $20-25$ times faster. Accordingly, we expect the Bezoutian method to become increasingly valuable for multiscale problems involving two-loop topologies with fewer propagators and at three loops and beyond (see e.g. ref. [51]).

We end this paper by suggesting interesting projects for future research. It is desirable to understand the nature of the contour constraints in complete detail. In particular, is it possible to fully determine constraints arising from integration-by-parts identities directly from the underlying algebraic geometry? Recent progress for the planar double box shows that discrete symmetries to some extent determine these constraints [49]. Such symmetries seem to be less constraining at higher genera. We also find it urgent to extend maximal unitarity to $D$ dimensions to recover terms missed in strictly $4 D$. Another very important next step is to extend the method to basis integrals with five external legs, for example the pentabox and turtle-box and the related nonplanar diagrams. First of all from a phenomenological point of view, but we also hope that a generalization beyond four external particles will offer insight in uniqueness of projectors [47]. We expect the one-mass 
hepta-cuts presented in this paper to be valuable in that direction, because octa-cuts may be evaluated as hepta-cuts followed by a particular choice of contour that puts the last propagator on-shell. Theoretically speaking, the master integral coefficients for all-massive six-point planar and nonplanar double boxes are exciting to compute because in those cases the on-shell parametrization is irrational and hence the maximally cut integrals suffer from genuine branch cuts [47]. Ultimately, it would be intriguing to implement the formalism numerically. We are looking forward to address some of these questions soon.

\section{Acknowledgments}

We have benefited from many discussions with Emil Bjerrum-Bohr, Simon Caron-Huot, Poul Henrik Damgaard, Rijun Huang, Henrik Johansson and David Kosower. A special thanks goes to Hjalte Frellesvig and Kasper Larsen for careful reading of the manuscript in draft stage and helpful comments. Both authors are grateful to Institut de Physique Théorique, CEA Saclay, and especially David Kosower for hospitality during stages of this project. The work of YZ is supported by Danish Council for Independent Research (FNU) grant 11-107241.

\section{A Explicit parametrization of hepta-cut solutions}

In this appendix we provide explicit forms of the loop momenta in all branches for the two distinct classes of hepta-cut solutions considered in the present paper. We also shed light on the pole structure.

Before we begin, recall that $\ell_{1}$ and $\ell_{2}$ are written in a basis of mutually flattened momenta and parametrized by the complex variables $\zeta_{i}, \xi_{i}$ and $\xi_{i}^{\prime}$,

$$
\begin{aligned}
\ell_{1}^{\mu}\left(\zeta_{i}, \xi_{i}, \xi_{i}^{\prime}\right)= & \zeta_{1} \eta_{1}^{\mu}+\xi_{1} \xi_{1}^{\prime} k_{1}^{b, \mu}+\xi_{2} \xi_{2}^{\prime} \frac{k_{1}^{b} \cdot k_{4}^{b}}{k_{2}^{b} \cdot k_{4}^{b}} k_{2}^{b, \mu} \\
& +\frac{\xi_{1} \xi_{2}^{\prime}}{2} \frac{\left[1^{b} 4^{b}\right]}{\left[2^{b} 4^{b}\right]}\left\langle 1^{b,-}\left|\gamma^{\mu}\right| 2^{b,-}\right\rangle+\frac{\xi_{2} \xi_{1}^{\prime}}{2} \frac{\left\langle 1^{b} 4^{b}\right\rangle}{\left\langle 2^{b} 4^{b}\right\rangle}\left\langle 2^{b,-}\left|\gamma^{\mu}\right| 1^{b,-}\right\rangle, \\
\ell_{2}^{\mu}\left(\zeta_{i}, \xi_{i}, \xi_{i}^{\prime}\right)= & \zeta_{2} \eta_{2}^{\mu}+\xi_{3} \xi_{3}^{\prime} \frac{k_{1}^{b} \cdot k_{4}^{b}}{k_{1}^{b} \cdot k_{3}^{b}} k_{3}^{b, \mu}+\xi_{4} \xi_{4}^{\prime} k_{4}^{b, \mu} \\
& +\frac{\xi_{3} \xi_{4}^{\prime}}{2} \frac{\left\langle 1^{b} 4^{b}\right\rangle}{\left\langle 1^{b} 3^{b}\right\rangle}\left\langle 3^{b,-}\left|\gamma^{\mu}\right| 4^{b,-}\right\rangle+\frac{\xi_{4} \xi_{3}^{\prime}}{2} \frac{\left[1^{b} 4^{b}\right]}{\left[1^{b} 3^{b}\right]}\left\langle 4^{b,-}\left|\gamma^{\mu}\right| 3^{b,-}\right\rangle .
\end{aligned}
$$

After imposing the constraints of the maximal cut we find two distinct classes of multiply degenerate kinematic solutions. In all cases, $\zeta_{1}=\zeta_{2}=0$. For the sake of completeness we list the expansions of all inverse propagators in parameter space with $\eta_{1}=k_{2}^{b}$ and $\eta_{2}=k_{4}^{b}$ 
utilized in the computation of maximally cut integrals.

$$
\begin{aligned}
& \ell_{1}^{2}=\gamma_{12} \zeta_{1} \xi_{1} \xi_{1}^{\prime} \\
& \ell_{2}^{2}=\gamma_{34} \frac{k_{1}^{b} \cdot k_{4}^{b}}{k_{1}^{b} \cdot k_{3}^{b}} \zeta_{2} \xi_{3} \xi_{3}^{\prime}, \\
& \left(\ell_{1}-k_{1}\right)^{2}=m_{1}^{2}+\gamma_{12} \zeta_{1} \xi_{1} \xi_{1}^{\prime}-\gamma_{12} \zeta_{1}-m_{1}^{2} \xi_{1} \xi_{1}^{\prime}-\gamma_{12} \frac{k_{1}^{b} \cdot k_{4}^{b}}{k_{2}^{b} \cdot k_{4}^{b}} \xi_{2} \xi_{2}^{\prime}, \\
& \left(\ell_{1}-k_{12}\right)^{2}=m_{1}^{2}+\gamma_{12} \zeta_{1} \xi_{1} \xi_{1}^{\prime}-\gamma_{12} \zeta_{1}-m_{1}^{2} \xi_{1} \xi_{1}^{\prime}-\gamma_{12} \frac{k_{1}^{b} \cdot k_{4}^{b}}{k_{2}^{b} \cdot k_{4}^{b}} \xi_{2} \xi_{2}^{\prime}, \\
& \left(\ell_{2}-k_{4}\right)^{2}=m_{4}^{2}+\gamma_{34} \frac{k_{1}^{b} \cdot k_{4}^{b}}{k_{1}^{b} \cdot k_{3}^{b}} \zeta_{2} \xi_{3} \xi_{3}^{\prime}-m_{4}^{2}\left(\zeta_{2}+\xi_{4} \xi_{4}^{\prime}\right)-\gamma_{34} \frac{k_{1}^{b} \cdot k_{4}^{b}}{k_{1}^{b} \cdot k_{3}^{b}} \xi_{3} \xi_{3}^{\prime}, \\
& \left(\ell_{1}+\ell_{2}\right)^{2}=\gamma_{12} \zeta_{1} \xi_{1} \xi_{1}^{\prime}+\gamma_{34} \frac{k_{1}^{b} \cdot k_{4}^{b}}{k_{1}^{b} \cdot k_{3}^{b}} \zeta_{2} \xi_{3} \xi_{3}^{\prime} \\
& +2 k_{1}^{b} \cdot k_{4}^{b}\left(\xi_{1}\left(\xi_{3}+\xi_{4}\right)+\xi_{2}\left(\tau \xi_{3}+\xi_{4}\right)\right) \times\left(\xi_{1}^{\prime}\left(\xi_{3}^{\prime}+\xi_{4}^{\prime}\right)+\xi_{2}^{\prime}\left(\tau \xi_{3}^{\prime}+\xi_{4}^{\prime}\right)\right) \text {, } \\
& \left(\ell_{1}+\ell_{2}+k_{3}\right)^{2}=m_{3}^{2}+\gamma_{12} \zeta_{1} \xi_{1} \xi_{1}^{\prime}+\gamma_{34} \frac{k_{1}^{b} \cdot k_{4}^{b}}{k_{1}^{b} \cdot k_{3}^{b}} \zeta_{2} \xi_{3} \xi_{3}^{\prime} \\
& +2 k_{1}^{b} \cdot k_{4}^{b}\left(\xi_{1}\left(\xi_{3}+\xi_{4}\right)+\xi_{2}\left(\tau \xi_{3}+\xi_{4}\right)\right) \times\left(\xi_{1}^{\prime}\left(\xi_{3}^{\prime}+\xi_{4}^{\prime}\right)+\xi_{2}^{\prime}\left(\tau \xi_{3}^{\prime}+\xi_{4}^{\prime}\right)\right) \\
& +2\left\{k_{1}^{b} \cdot k_{3} \xi_{1} \xi_{1}^{\prime}+\frac{m_{3}^{2} k_{1}^{b} \cdot k_{4}^{b}}{2 k_{1}^{b} \cdot k_{3}^{b}} \xi_{3} \xi_{3}^{\prime}+\frac{\gamma_{34}}{2} \xi_{4} \xi_{4}^{\prime}\right. \\
& \left.+\left[\tau k_{1}^{b} \cdot k_{3}^{b}+\frac{m_{3}^{2} k_{1}^{b} \cdot k_{4}^{b}}{\gamma_{34}}\right]\left(\xi_{1} \xi_{2}^{\prime}+\xi_{1}^{\prime} \xi_{2}\right)+\frac{k_{1}^{b} \cdot k_{4}^{b} k_{2}^{b} \cdot k_{3}}{k_{2}^{b} \cdot k_{4}^{b}} \xi_{2} \xi_{2}^{\prime}\right\} \text {. }
\end{aligned}
$$

\section{A.1 The four-mass case and smooth massless limits}

The first class of solutions to the hepta-cut equations covers the four-mass case but also applies in the limit where one or two external legs in the crossed end of the diagram become massless. We simplify expressions by virtue of introducing a new variable $w(z)$ defined by

$$
w(z)=\frac{1+z}{1+\tau z} \Longleftrightarrow z(w)=-\frac{1-w}{1-\tau w} .
$$

Then we have the following six inequivalent solutions,

$$
\begin{aligned}
\left.\ell_{1}^{\mu}\right|_{\mathcal{S}_{1}}= & \bar{\xi}_{1} k_{1}^{b, \mu}+\bar{\xi}_{2} \frac{k_{1}^{b} \cdot k_{4}^{b}}{k_{2}^{b} \cdot k_{4}^{b}} k_{2}^{b, \mu}+\frac{\bar{\xi}_{2}}{2 z} \frac{\left[1^{b} 4^{b}\right]}{\left[2^{b} 4^{b}\right]}\left\langle 1^{b,-}\left|\gamma^{\mu}\right| 2^{b,-}\right\rangle+\frac{\bar{\xi}_{1} z}{2} \frac{\left\langle 1^{b} 4^{b}\right\rangle}{\left\langle 2^{b} 4^{b}\right\rangle}\left\langle 2^{b,-}\left|\gamma^{\mu}\right| 1^{b,-}\right\rangle, \\
\left.\ell_{2}^{\mu}\right|_{\mathcal{S}_{1}}= & -\frac{1+\tau z}{(1-\tau)\left(\bar{\xi}_{1}-\tau \bar{\xi}_{2}\right) z}\left\{\mu \tau w(z)\left(\bar{\xi}_{1} z+\bar{\xi}_{2}\right) \frac{k_{1}^{b} \cdot k_{4}^{b}}{k_{1}^{b} \cdot k_{3}^{b}} k_{3}^{b, \mu}-\left(\bar{\xi}_{1} z+\tau \bar{\xi}_{2}\right) k_{4}^{b, \mu}\right. \\
& \left.-\frac{1}{2} \mu \tau w(z)\left(\bar{\xi}_{1} z+\tau \bar{\xi}_{2}\right) \frac{\left\langle 1^{b} 4^{b}\right\rangle}{\left\langle 1^{b} 3^{b}\right\rangle}\left\langle 3^{b,-}\left|\gamma^{\mu}\right| 4^{b,-}\right\rangle+\frac{1}{2}\left(\bar{\xi}_{1} z+\bar{\xi}_{2}\right) \frac{\left[1^{b} 4^{b}\right]}{\left[1^{b} 3^{b}\right]}\left\langle 4^{b,-}\left|\gamma^{\mu}\right| 3^{b,-}\right\rangle\right\}, \\
\left.\ell_{1}^{\mu}\right|_{\mathcal{S}_{2}}= & \bar{\xi}_{1} k_{1}^{b, \mu}+\bar{\xi}_{2} \frac{k_{1}^{b} \cdot k_{4}^{b}}{k_{2}^{b} \cdot k_{4}^{b}} k_{2}^{b, \mu}+\frac{\bar{\xi}_{1} z}{2} \frac{\left[1^{b} 4^{b}\right]}{\left[2^{b} 4^{b}\right]}\left\langle 1^{b,-}\left|\gamma^{\mu}\right| 2^{b,-}\right\rangle+\frac{\bar{\xi}_{2}}{2 z} \frac{\left\langle 1^{b} 4^{b}\right\rangle}{\left\langle 2^{b} 4^{b}\right\rangle}\left\langle 2^{b,-}\left|\gamma^{\mu}\right| 1^{b,-}\right\rangle,
\end{aligned}
$$




$$
\begin{aligned}
\left.\ell_{2}^{\mu}\right|_{\mathcal{S}_{2}=} & -\frac{1+\tau z}{(1-\tau)\left(\bar{\xi}_{1}-\tau \bar{\xi}_{2}\right) z}\left\{\mu \tau w(z)\left(\bar{\xi}_{1} z+\bar{\xi}_{2}\right) \frac{k_{1}^{b} \cdot k_{4}^{b}}{k_{1}^{b} \cdot k_{3}^{b}} k_{3}^{b, \mu}-\left(\bar{\xi}_{1} z+\tau \bar{\xi}_{2}\right) k_{4}^{b, \mu}\right. \\
& \left.-\frac{1}{2} \mu \tau w(z)\left(\bar{\xi}_{1} z+\tau \bar{\xi}_{2}\right) \frac{\left\langle 1^{b} 4^{b}\right\rangle}{\left\langle 1^{b} 3^{b}\right\rangle}\left\langle 3^{b,-}\left|\gamma^{\mu}\right| 4^{b,-}\right\rangle+\frac{1}{2}\left(\bar{\xi}_{1} z+\bar{\xi}_{2}\right) \frac{\left[1^{b} 4^{b}\right]}{\left[1^{b} 3^{b}\right]}\left\langle 4^{b,-}\left|\gamma^{\mu}\right| 3^{b,-}\right\rangle\right\} \\
\left.\ell_{1}^{\mu}\right|_{\mathcal{S}_{3}=}= & \bar{\xi}_{1} k_{1}^{b, \mu}+\bar{\xi}_{2} \frac{k_{1}^{b} \cdot k_{4}^{b}}{k_{2}^{b} \cdot k_{4}^{b}} k_{2}^{b, \mu}-\frac{\bar{\xi}_{1}}{2} \frac{\left[1^{b} 4^{b}\right]}{\left[2^{b} 4^{b}\right]}\left\langle 1^{b,-}\left|\gamma^{\mu}\right| 2^{b,-}\right\rangle-\frac{\bar{\xi}_{2}}{2} \frac{\left\langle 1^{b} 4^{b}\right\rangle}{\left\langle 2^{b} 4^{b}\right\rangle}\left\langle 2^{b,-}\left|\gamma^{\mu}\right| 1^{b,-}\right\rangle \\
\left.\ell_{2}^{\mu}\right|_{\mathcal{S}_{3}}= & k_{4}^{b, \mu}+\frac{z}{2} \frac{\left\langle 1^{b} 4^{b}\right\rangle}{\left\langle 1^{b} 3^{b}\right\rangle}\left\langle 3^{b,-}\left|\gamma^{\mu}\right| 4^{b,-}\right\rangle \\
\left.\ell_{1}^{\mu}\right|_{\mathcal{S}_{4}}= & \bar{\xi}_{1} k_{1}^{b, \mu}+\bar{\xi}_{2} \frac{k_{1}^{b} \cdot k_{4}^{b}}{k_{2}^{b} \cdot k_{4}^{b}} k_{2}^{b, \mu}-\frac{\bar{\xi}_{2}}{2} \frac{\left[1^{b} 4^{b}\right]}{\left[2^{b} 4^{b}\right]}\left\langle 1^{b,-}\left|\gamma^{\mu}\right| 2^{b,-}\right\rangle-\frac{\bar{\xi}_{1}}{2} \frac{\left\langle 1^{b} 4^{b}\right\rangle}{\left\langle 2^{b} 4^{b}\right\rangle}\left\langle 2^{b,-}\left|\gamma^{\mu}\right| 1^{b,-}\right\rangle \\
\left.\ell_{2}^{\mu}\right|_{\mathcal{S}_{4}}= & k_{4}^{b, \mu}+\frac{z}{2} \frac{\left[1^{b} 4^{b}\right]}{\left[1^{b} 3^{b}\right]}\left\langle 4^{b,-}\left|\gamma^{\mu}\right| 3^{b,-}\right\rangle, \\
\left.\ell_{1}^{\mu}\right|_{\mathcal{S}_{5}=} & \bar{\xi}_{1} k_{1}^{b, \mu}+\bar{\xi}_{2} \frac{k_{1}^{b} \cdot k_{4}^{b}}{k_{2}^{b} \cdot k_{4}^{b}} k_{2}^{b, \mu}-\frac{\bar{\xi}_{1}}{2 \tau} \frac{\left[1^{b} 4^{b}\right]}{\left[2^{b} 4^{b}\right]}\left\langle 1^{b,-}\left|\gamma^{\mu}\right| 2^{b,-}\right\rangle-\frac{\bar{\xi}_{2} \tau}{2} \frac{\left\langle 1^{b} 4^{b}\right\rangle}{\left\langle 2^{b} 4^{b}\right\rangle}\left\langle 2^{b,-}\left|\gamma^{\mu}\right| 1^{b,-}\right\rangle \\
\left.\ell_{2}^{\mu}\right|_{\mathcal{S}_{5}}= & \mu \frac{k_{1}^{b} \cdot k_{4}^{b}}{k_{1}^{b} \cdot k_{3}^{b}} k_{3}^{b, \mu}+\frac{z}{2} \frac{\left.11^{b} 4^{b}\right]}{\left[1^{b} 3^{b}\right]}\left\langle 4^{b,-}\left|\gamma^{\mu}\right| 3^{b,-}\right\rangle, \\
\left.\ell_{1}^{\mu}\right|_{\mathcal{S}_{6}}= & \bar{\xi}_{1} k_{1}^{b, \mu}+\bar{\xi}_{2} \frac{k_{1}^{b} \cdot k_{4}^{b}}{k_{2}^{b} \cdot k_{4}^{b}} k_{2}^{b, \mu}-\frac{\tau \bar{\xi}_{2}}{2} \frac{\left[1^{b} 4^{b}\right]}{\left[2^{b} 4^{b}\right]}\left\langle 1^{b,-}\left|\gamma^{\mu}\right| 2^{b,-}\right\rangle-\frac{\bar{\xi}_{1}}{2 \tau} \frac{\left\langle 1^{b} 4^{b}\right\rangle}{\left\langle 2^{b} 4^{b}\right\rangle}\left\langle 2^{b,-}\left|\gamma^{\mu}\right| 1^{b,-}\right\rangle \\
\left.\ell_{2}^{\mu}\right|_{\mathcal{S}_{6}}= & \mu \frac{k_{1}^{b} \cdot k_{4}^{b}}{k_{1}^{b} \cdot k_{3}^{b}} k_{3}^{b, \mu}+\frac{z}{2} \frac{\left\langle 1^{b} 4^{b}\right\rangle}{\left\langle 1^{b} 3^{b}\right\rangle}\left\langle 3^{b,-}\left|\gamma^{\mu}\right| 4^{b,-}\right\rangle .
\end{aligned}
$$

The displayed kinematic constants were defined in eqs. (3.30)-(3.32) in the main text. It follows that solutions $\mathcal{S}_{1}$ and $\mathcal{S}_{2}$ give rise to a singularity in both the left and right loop momentum when $z \rightarrow 0$, whereas $\mathcal{S}_{3}$ through $\mathcal{S}_{6}$ have no poles for finite values of $z$ and thus produce holomorphic integrands insertions.

\section{A.2 Degenerate massless limits}

As explained in the body of this paper, $\bar{\xi}_{2}=0$ for $m_{1} m_{2}=0$ and for this class of kinematics the zero locus of the inverse propagators decomposes into a union of eight irreducible components. Moreover, we have $\bar{\xi}_{1}=1-m_{1}^{2} / s_{12}$ along with various other simplifications which we do not show here in detail. Explicitly,

$$
\begin{aligned}
\left.\ell_{1}^{\mu}\right|_{\widetilde{\mathcal{S}}_{1}}=\left(1-m_{1}^{2} / s_{12}\right) & \left\{k_{1}^{b, \mu}+\frac{z}{2} \frac{\left\langle 1^{b} 4^{b}\right\rangle}{\left\langle 2^{b} 4^{b}\right\rangle}\left\langle 2^{b,-}\left|\gamma^{\mu}\right| 1^{b,-}\right\rangle\right\}, \\
\left.\ell_{2}^{\mu}\right|_{\widetilde{\mathcal{S}}_{1}}=-\frac{1+\tau z}{1-\tau}\left\{\mu \tau w(z) \frac{k_{1}^{b} \cdot k_{4}^{b}}{k_{1}^{b} \cdot k_{3}^{b}} k_{3}^{b, \mu}-k_{4}^{b, \mu}\right. & \\
& \left.\quad \frac{\mu \tau w(z)}{2} \frac{\left\langle 1^{b} 4^{b}\right\rangle}{\left\langle 1^{b} 3^{b}\right\rangle}\left\langle 3^{b,-}\left|\gamma^{\mu}\right| 4^{b,-}\right\rangle-\frac{\left[1^{b} 4^{b}\right]}{\left[1^{b} 3^{b}\right]}\left\langle 4^{b,-}\left|\gamma^{\mu}\right| 3^{b,-}\right\rangle\right\}
\end{aligned}
$$




$$
\begin{aligned}
& \left.\ell_{1}^{\mu}\right|_{\mathcal{S}_{2}}=\left(1-m_{1}^{2} / s_{12}\right)\left\{k_{1}^{b, \mu}+\frac{z}{2} \frac{\left[1^{b} 4^{b}\right]}{\left[2^{b} 4^{b}\right]}\left\langle 1^{b,-}\left|\gamma^{\mu}\right| 2^{b,-}\right\rangle\right\} \\
& \left.\ell_{2}^{\mu}\right|_{\mathcal{S}_{2}}=-\frac{1+\tau z}{1-\tau}\left\{\mu \tau w(z) \frac{k_{1}^{b} \cdot k_{4}^{b}}{k_{1}^{b} \cdot k_{3}^{b}} k_{3}^{b, \mu}-k_{4}^{b, \mu}\right. \\
& \left.+\frac{\left\langle 1^{b} 4^{b}\right\rangle}{\left\langle 1^{b} 3^{b}\right\rangle}\left\langle 3^{b,-}\left|\gamma^{\mu}\right| 4^{b,-}\right\rangle+\frac{\mu \tau w(z)}{2} \frac{\left[1^{b} 4^{b}\right]}{\left[1^{b} 3^{b}\right]}\left\langle 4^{b,-}\left|\gamma^{\mu}\right| 3^{b,-}\right\rangle\right\}, \\
& \left.\ell_{1}^{\mu}\right|_{\widetilde{\mathcal{S}}_{3}}=\left(1-m_{1}^{2} / s_{12}\right)\left\{k_{1}^{b, \mu}+\frac{z}{2} \frac{\left\langle 1^{b} 4^{b}\right\rangle}{\left\langle 2^{b} 4^{b}\right\rangle}\left\langle 2^{b,-}\left|\gamma^{\mu}\right| 1^{b,-}\right\rangle\right\}, \\
& \left.\ell_{2}^{\mu}\right|_{\widetilde{\mathcal{S}}_{3}}=-\frac{1+\tau z}{1-\tau}\left\{\mu \tau w(z) \frac{k_{1}^{b} \cdot k_{4}^{b}}{k_{1}^{b} \cdot k_{3}^{b}} k_{3}^{b, \mu}-k_{4}^{b, \mu}\right. \\
& \left.-\frac{\mu \tau}{2} \frac{\left\langle 1^{b} 4^{b}\right\rangle}{\left\langle 1^{b} 3^{b}\right\rangle}\left\langle 3^{b,-}\left|\gamma^{\mu}\right| 4^{b,-}\right\rangle+\frac{w(z)}{2} \frac{\left[1^{b} 4^{b}\right]}{\left[1^{b} 3^{b}\right]}\left\langle 4^{b,-}\left|\gamma^{\mu}\right| 3^{b,-}\right\rangle\right\}, \\
& \left.\ell_{1}^{\mu}\right|_{\widetilde{\mathcal{S}}_{4}}=\left(1-m_{1}^{2} / s_{12}\right)\left\{k_{1}^{b, \mu}+\frac{z}{2} \frac{\left\langle 1^{b} 4^{b}\right\rangle}{\left\langle 2^{b} 4^{b}\right\rangle}\left\langle 2^{b,-}\left|\gamma^{\mu}\right| 1^{b,-}\right\rangle\right\} \\
& \left.\ell_{2}^{\mu}\right|_{\widetilde{\mathcal{S}}_{4}}=-\frac{1+\tau z}{1-\tau}\left\{\mu \tau w(z) \frac{k_{1}^{b} \cdot k_{4}^{b}}{k_{1}^{b} \cdot k_{3}^{b}} k_{3}^{b, \mu}-k_{4}^{b, \mu}\right. \\
& \left.+\frac{w(z)}{2} \frac{\left\langle 1^{b} 4^{b}\right\rangle}{\left\langle 1^{b} 3^{b}\right\rangle}\left\langle 3^{b,-}\left|\gamma^{\mu}\right| 4^{b,-}\right\rangle-\frac{\mu \tau}{2} \frac{\left[1^{b} 4^{b}\right]}{\left[1^{b} 3^{b}\right]}\left\langle 4^{b,-}\left|\gamma^{\mu}\right| 3^{b,-}\right\rangle\right\}, \\
& \left.\ell_{1}^{\mu}\right|_{\widetilde{\mathcal{S}}_{5}}=\left(1-m_{1}^{2} / s 12\right)\left\{k_{1}^{b, \mu}-\frac{1}{2} \frac{\left[1^{b} 4^{b}\right]}{\left[2^{b} 4^{b}\right]}\left\langle 1^{b,-}\left|\gamma^{\mu}\right| 2^{b,-}\right\rangle\right\}, \\
& \left.\ell_{2}^{\mu}\right|_{\widetilde{\mathcal{S}}_{5}}=k_{4}^{b, \mu}+\frac{z}{2} \frac{\left\langle 1^{b} 4^{b}\right\rangle}{\left\langle 1^{b} 3^{b}\right\rangle}\left\langle 3^{b,-}\left|\gamma^{\mu}\right| 4^{b,-}\right\rangle, \\
& \ell_{1}^{\mu}{\mid \mathcal{S}_{6}}=\left(1-m_{1}^{2} / s_{12}\right)\left\{k_{1}^{b, \mu}-\frac{1}{2} \frac{\left\langle 1^{b} 4^{b}\right\rangle}{\left\langle 2^{b} 4^{b}\right\rangle}\left\langle 2^{b,-}\left|\gamma^{\mu}\right| 1^{b,-}\right\rangle\right\}, \\
& \left.\ell_{2}^{\mu}\right|_{\widetilde{\mathcal{S}}_{6}}=k_{4}^{b, \mu}+\frac{z}{2} \frac{\left[1^{b} 4^{b}\right]}{\left[1^{b} 3^{b}\right]}\left\langle 4^{b,-}\left|\gamma^{\mu}\right| 3^{b,-}\right\rangle \\
& \left.\ell_{1}^{\mu}\right|_{\widetilde{\mathcal{S}}_{7}}=\left(1-m_{1}^{2} / s_{12}\left\{k_{1}^{b, \mu}-\frac{\bar{\xi}_{1}}{2 \tau} \frac{\left[1^{b} 4^{b}\right]}{\left[2^{b} 4^{b}\right]}\left\langle 1^{b,-}\left|\gamma^{\mu}\right| 2^{b,-}\right\rangle\right\},\right. \\
& \left.\ell_{2}^{\mu}\right|_{\widetilde{\mathcal{S}}_{7}}=\mu \frac{k_{1}^{b} \cdot k_{4}^{b}}{k_{1}^{b} \cdot k_{3}^{b}} k_{3}^{b, \mu}+\frac{z}{2} \frac{\left[1^{b} 4^{b}\right]}{\left[1^{b} 3^{b}\right]}\left\langle 4^{b,-}\left|\gamma^{\mu}\right| 3^{b,-}\right\rangle, \\
& \left.\ell_{1}^{\mu}\right|_{\widetilde{\mathcal{S}}_{8}}=\left(1-m_{1}^{2} / s_{12}\right)\left\{k_{1}^{b, \mu}-\frac{\bar{\xi}_{1}}{2 \tau} \frac{\left\langle 1^{b} 4^{b}\right\rangle}{\left\langle 2^{b} 4^{b}\right\rangle}\left\langle 2^{b,-}\left|\gamma^{\mu}\right| 1^{b,-}\right\rangle\right\}, \\
& \left.\ell_{2}^{\mu}\right|_{\widetilde{\mathcal{S}}_{8}}=\mu \frac{k_{1}^{b} \cdot k_{4}^{b}}{k_{1}^{b} \cdot k_{3}^{b}} k_{3}^{b, \mu}+\frac{z}{2} \frac{\left\langle 1^{b} 4^{b}\right\rangle}{\left\langle 1^{b} 3^{b}\right\rangle}\left\langle 3^{b,-}\left|\gamma^{\mu}\right| 4^{b,-}\right\rangle \text {. }
\end{aligned}
$$

\section{B Structure of global poles}

With the discussion of the previous section in mind, we explain that the global poles are inherited through chiral branchings. To that end, define the following eight Jacobian global 
poles in class (a),

$$
\begin{aligned}
& \mathcal{G}_{1} \equiv \mathcal{S}_{1} \cap S_{4}, \quad \mathcal{G}_{2} \equiv \mathcal{S}_{1} \cap S_{6}, \quad \mathcal{G}_{3} \equiv \mathcal{S}_{1} \cap S_{3}, \quad \mathcal{G}_{4} \equiv \mathcal{S}_{1} \cap S_{5}, \\
& \mathcal{G}_{5} \equiv \mathcal{S}_{2} \cap S_{3}, \quad \mathcal{G}_{6} \equiv \mathcal{S}_{2} \cap S_{5}, \quad \mathcal{G}_{7} \equiv \mathcal{S}_{2} \cap S_{4}, \quad \mathcal{G}_{8} \equiv \mathcal{S}_{2} \cap S_{6},
\end{aligned}
$$

along with the additional poles in numerator insertions,

$$
\begin{aligned}
& \left.\mathcal{G}_{5} \equiv \mathcal{S}_{1}\right|_{z=0},\left.\quad \mathcal{G}_{10} \equiv \mathcal{S}_{1}\right|_{z=0},\left.\quad \mathcal{G}_{11} \equiv \mathcal{S}_{1}\right|_{z=\infty},\left.\quad \mathcal{G}_{12} \equiv \mathcal{S}_{2}\right|_{z=\infty}, \\
& \left.\mathcal{G}_{13} \equiv \mathcal{S}_{3}\right|_{z=\infty},\left.\quad \mathcal{G}_{14} \equiv \mathcal{S}_{4}\right|_{z=\infty},\left.\quad \mathcal{G}_{15} \equiv \mathcal{S}_{5}\right|_{z=\infty},\left.\quad \mathcal{G}_{16} \equiv \mathcal{S}_{6}\right|_{z=\infty}
\end{aligned}
$$

As the total sum of residues for a meromorphic differential form on $\mathbb{C P}^{1}$ vanishes, only ten residues evaluated at, say, $\left\{\mathcal{G}_{1}, \ldots, \mathcal{G}_{10}\right\}$ are independent.

Moreover, in class (b) we define the global poles

$$
\begin{aligned}
& \mathcal{G}_{1}^{\prime} \equiv \mathcal{S}_{1}^{\prime} \cap S_{6}^{\prime}, \quad \mathcal{G}_{2}^{\prime} \equiv \mathcal{S}_{1}^{\prime} \cap S_{8}^{\prime}, \quad \mathcal{G}_{3}^{\prime} \equiv \mathcal{S}_{3}^{\prime} \cap S_{5}^{\prime}, \quad \mathcal{G}_{4}^{\prime} \equiv \mathcal{S}_{3}^{\prime} \cap S_{7}^{\prime}, \quad \mathcal{G}_{5}^{\prime} \equiv \mathcal{S}_{1}^{\prime} \cap S_{3}^{\prime}, \\
& \mathcal{G}_{6}^{\prime} \equiv \mathcal{S}_{2}^{\prime} \cap S_{5}^{\prime}, \quad \mathcal{G}_{7}^{\prime} \equiv \mathcal{S}_{2}^{\prime} \cap S_{7}^{\prime}, \quad \mathcal{G}_{8}^{\prime} \equiv \mathcal{S}_{4}^{\prime} \cap S_{6}^{\prime}, \quad \mathcal{G}_{9}^{\prime} \equiv \mathcal{S}_{4}^{\prime} \cap S_{8}^{\prime}, \quad \mathcal{G}_{10^{\prime}} \equiv \mathcal{S}_{2}^{\prime} \cap S_{4}^{\prime} .
\end{aligned}
$$

All primed branches are obviously holomorphically parametrized and hence none of them have singularities for additional finite values of $z$. However, there are possibly eight residues at infinity,

$$
\begin{aligned}
& \left.\widetilde{\mathcal{G}}_{11} \equiv \mathcal{S}_{1}\right|_{z=\infty},\left.\quad \widetilde{\mathcal{G}}_{12} \equiv \mathcal{S}_{2}\right|_{z=\infty},\left.\quad \widetilde{\mathcal{G}}_{13} \equiv \mathcal{S}_{3}\right|_{z=\infty},\left.\quad \widetilde{\mathcal{G}}_{14} \equiv \mathcal{S}_{4}\right|_{z=\infty}, \\
& \left.\widetilde{\mathcal{G}}_{15} \equiv \mathcal{S}_{5}\right|_{z=\infty},\left.\quad \widetilde{\mathcal{G}}_{16} \equiv \mathcal{S}_{6}\right|_{z=\infty},\left.\quad \widetilde{\mathcal{G}}_{17} \equiv \mathcal{S}_{7}\right|_{z=\infty},\left.\quad \widetilde{\mathcal{G}}_{18} \equiv \mathcal{S}_{8}\right|_{z=\infty} .
\end{aligned}
$$

At this stage, it is not hard to realize that the eight Jacobian poles in class (a) are inherited by class (b). The map goes as follows,

$$
\left(\mathcal{G}_{1}, \mathcal{G}_{2}, \mathcal{G}_{3}, \mathcal{G}_{4}, \mathcal{G}_{6}, \mathcal{G}_{7}, \mathcal{G}_{8}, \mathcal{G}_{9}\right) \stackrel{\bar{\xi}_{2} \rightarrow 0}{\longrightarrow}\left(\mathcal{G}_{1}, \mathcal{G}_{2}, \mathcal{G}_{3}, \mathcal{G}_{4}, \mathcal{G}_{6}, \mathcal{G}_{7}, \mathcal{G}_{8}, \mathcal{G}_{9}\right)
$$

and similarly for the residues at $z=0$ and $z=\infty$,

$$
\left(\mathcal{G}_{11}, \mathcal{G}_{12}, \mathcal{G}_{5}, \mathcal{G}_{10}, \mathcal{G}_{13}, \mathcal{G}_{14}, \mathcal{G}_{15}, \mathcal{G}_{16}\right) \stackrel{\bar{\xi}_{2} \rightarrow 0}{\longrightarrow}\left(\widetilde{\mathcal{G}}_{11}, \widetilde{\mathcal{G}}_{12}, \widetilde{\mathcal{G}}_{13}, \widetilde{\mathcal{G}}_{14}, \widetilde{\mathcal{G}}_{15}, \widetilde{\mathcal{G}}_{16}, \widetilde{\mathcal{G}}_{17}, \widetilde{\mathcal{G}}_{18}\right) .
$$

The remaining two Jacobian global poles $\widetilde{\mathcal{G}}_{5}$ and $\widetilde{\mathcal{G}}_{10}$ are located at the nodal points $\mathcal{S}_{1} \cap \mathcal{S}_{3}$ and $\mathcal{S}_{2} \cap \mathcal{S}_{4}$ respectively, and are thus generated by chiral branching from $6 \rightarrow 8$ hepta-cut solutions. By the one-dimensional Global Residue Theorem, the number of independent residues is clearly invariant.

Open Access. This article is distributed under the terms of the Creative Commons Attribution License (CC-BY 4.0), which permits any use, distribution and reproduction in any medium, provided the original author(s) and source are credited.

\section{References}

[1] Z. Bern, L.J. Dixon, D.C. Dunbar and D.A. Kosower, Fusing gauge theory tree amplitudes into loop amplitudes, Nucl. Phys. B 435 (1995) 59 [hep-ph/9409265] [INSPIRE]. 
[2] Z. Bern, L.J. Dixon, D.C. Dunbar and D.A. Kosower, One loop $n$ point gauge theory amplitudes, unitarity and collinear limits, Nucl. Phys. B 425 (1994) 217 [hep-ph/9403226] [INSPIRE].

[3] R. Britto, F. Cachazo and B. Feng, New recursion relations for tree amplitudes of gluons, Nucl. Phys. B 715 (2005) 499 [hep-th/0412308] [INSPIRE].

[4] R. Britto, F. Cachazo, B. Feng and E. Witten, Direct proof of tree-level recursion relation in Yang-Mills theory, Phys. Rev. Lett. 94 (2005) 181602 [hep-th/0501052] [InSPIRE].

[5] Z. Bern and A.G. Morgan, Massive loop amplitudes from unitarity, Nucl. Phys. B 467 (1996) 479 [hep-ph/9511336] [INSPIRE].

[6] Z. Bern, L.J. Dixon and D.A. Kosower, One loop amplitudes for $e^{+} e^{-}$to four partons, Nucl. Phys. B 513 (1998) 3 [hep-ph/9708239] [INSPIRE].

[7] R. Britto, F. Cachazo and B. Feng, Generalized unitarity and one-loop amplitudes in $N=4$ super-Yang-Mills, Nucl. Phys. B 725 (2005) 275 [hep-th/0412103] [INSPIRE].

[8] R. Britto, F. Cachazo and B. Feng, Computing one-loop amplitudes from the holomorphic anomaly of unitarity cuts, Phys. Rev. D 71 (2005) 025012 [hep-th/0410179] [INSPIRE].

[9] Z. Bern, N.E.J. Bjerrum-Bohr, D.C. Dunbar and H. Ita, Recursive calculation of one-loop QCD integral coefficients, JHEP 11 (2005) 027 [hep-ph/0507019] [INSPIRE].

[10] S.J. Bidder, N.E.J. Bjerrum-Bohr, D.C. Dunbar and W.B. Perkins, One-loop gluon scattering amplitudes in theories with $N<4$ supersymmetries, Phys. Lett. B 612 (2005) 75 [hep-th/0502028] [INSPIRE].

[11] R. Britto, E. Buchbinder, F. Cachazo and B. Feng, One-loop amplitudes of gluons in SQCD, Phys. Rev. D 72 (2005) 065012 [hep-ph/0503132] [InSPIRE].

[12] R. Britto, B. Feng and P. Mastrolia, The Cut-constructible part of QCD amplitudes, Phys. Rev. D 73 (2006) 105004 [hep-ph/0602178] [INSPIRE].

[13] P. Mastrolia, On Triple-cut of scattering amplitudes, Phys. Lett. B 644 (2007) 272 [hep-th/0611091] [INSPIRE].

[14] A. Brandhuber, S. McNamara, B.J. Spence and G. Travaglini, Loop amplitudes in pure Yang-Mills from generalised unitarity, JHEP 10 (2005) 011 [hep-th/0506068] [INSPIRE].

[15] G. Ossola, C.G. Papadopoulos and R. Pittau, Reducing full one-loop amplitudes to scalar integrals at the integrand level, Nucl. Phys. B 763 (2007) 147 [hep-ph/0609007] [INSPIRE].

[16] C. Anastasiou, R. Britto, B. Feng, Z. Kunszt and P. Mastrolia, Unitarity cuts and Reduction to master integrals in d dimensions for one-loop amplitudes, JHEP 03 (2007) 111 [hep-ph/0612277] [INSPIRE].

[17] Z. Bern, L.J. Dixon and D.A. Kosower, On-Shell Methods in Perturbative QCD, Annals Phys. 322 (2007) 1587 [arXiv:0704.2798] [INSPIRE].

[18] D. Forde, Direct extraction of one-loop integral coefficients, Phys. Rev. D 75 (2007) 125019 [arXiv:0704.1835] [INSPIRE].

[19] S.D. Badger, Direct Extraction Of One Loop Rational Terms, JHEP 01 (2009) 049 [arXiv:0806.4600] [INSPIRE].

[20] W.T. Giele, Z. Kunszt and K. Melnikov, Full one-loop amplitudes from tree amplitudes, JHEP 04 (2008) 049 [arXiv: 0801.2237] [InSPIRE]. 
[21] R. Britto and B. Feng, Unitarity cuts with massive propagators and algebraic expressions for coefficients, Phys. Rev. D 75 (2007) 105006 [hep-ph/0612089] [INSPIRE].

[22] R. Britto and B. Feng, Integral coefficients for one-loop amplitudes, JHEP 02 (2008) 095 [arXiv: 0711.4284] [INSPIRE].

[23] Z. Bern, J.J. Carrasco, T. Dennen, Y.-t. Huang and H. Ita, Generalized Unitarity and Six-Dimensional Helicity, Phys. Rev. D 83 (2011) 085022 [arXiv:1010.0494] [INSPIRE].

[24] C. Anastasiou, R. Britto, B. Feng, Z. Kunszt and P. Mastrolia, D-dimensional unitarity cut method, Phys. Lett. B 645 (2007) 213 [hep-ph/0609191] [INSPIRE].

[25] P. Mastrolia, Double-Cut of Scattering Amplitudes and Stokes' Theorem, Phys. Lett. B 678 (2009) 246 [arXiv:0905.2909] [INSPIRE].

[26] R.K. Ellis, W.T. Giele and Z. Kunszt, A Numerical Unitarity Formalism for Evaluating One-Loop Amplitudes, JHEP 03 (2008) 003 [arXiv:0708.2398] [INSPIRE].

[27] C.F. Berger, Z. Bern, L.J. Dixon, F. Febres Cordero, D. Forde et al., An Automated Implementation of On-Shell Methods for One-Loop Amplitudes, Phys. Rev. D 78 (2008) 036003 [arXiv: 0803.4180] [INSPIRE].

[28] G. Ossola, C.G. Papadopoulos and R. Pittau, CutTools: A Program implementing the OPP reduction method to compute one-loop amplitudes, JHEP 03 (2008) 042 [arXiv:0711.3596] [INSPIRE].

[29] P. Mastrolia, G. Ossola, C.G. Papadopoulos and R. Pittau, Optimizing the Reduction of One-Loop Amplitudes, JHEP 06 (2008) 030 [arXiv:0803.3964] [INSPIRE].

[30] W.T. Giele and G. Zanderighi, On the Numerical Evaluation of One-Loop Amplitudes: The Gluonic Case, JHEP 06 (2008) 038 [arXiv: 0805.2152] [inSPIRE].

[31] C.F. Berger, Z. Bern, L.J. Dixon, F. Febres Cordero, D. Forde et al., Precise Predictions for $W+3$ Jet Production at Hadron Colliders, Phys. Rev. Lett. 102 (2009) 222001 [arXiv:0902.2760] [INSPIRE].

[32] S. Badger, B. Biedermann and P. Uwer, NGluon: A Package to Calculate One-loop Multi-gluon Amplitudes, Comput. Phys. Commun. 182 (2011) 1674 [arXiv:1011.2900] [INSPIRE].

[33] C.F. Berger, Z. Bern, L.J. Dixon, F. Febres Cordero, D. Forde et al., Precise Predictions for $W+4$ Jet Production at the Large Hadron Collider, Phys. Rev. Lett. 106 (2011) 092001 [arXiv: 1009.2338] [INSPIRE].

[34] V. Hirschi, R. Frederix, S. Frixione, M.V. Garzelli, F. Maltoni et al., Automation of one-loop QCD corrections, JHEP 05 (2011) 044 [arXiv: 1103.0621] [INSPIRE].

[35] Z. Bern, J.S. Rozowsky and B. Yan, Two loop four gluon amplitudes in $N=4$ super Yang-Mills, Phys. Lett. B 401 (1997) 273 [hep-ph/9702424] [INSPIRE].

[36] Z. Bern, L.J. Dixon and D.A. Kosower, A Two loop four gluon helicity amplitude in QCD, JHEP 01 (2000) 027 [hep-ph/0001001] [INSPIRE].

[37] E.W.N. Glover, C. Oleari and M.E. Tejeda-Yeomans, Two loop QCD corrections to gluon-gluon scattering, Nucl. Phys. B 605 (2001) 467 [hep-ph/0102201] [INSPIRE].

[38] Z. Bern, A. De Freitas and L.J. Dixon, Two loop helicity amplitudes for gluon-gluon scattering in QCD and supersymmetric Yang-Mills theory, JHEP 03 (2002) 018 [hep-ph/0201161] [INSPIRE]. 
[39] C. Anastasiou, E.W.N. Glover, C. Oleari and M.E. Tejeda-Yeomans, Two-loop QCD corrections to the scattering of massless distinct quarks, Nucl. Phys. B 601 (2001) 318 [hep-ph/0010212] [INSPIRE].

[40] C. Anastasiou, E.W.N. Glover, C. Oleari and M.E. Tejeda-Yeomans, Two loop QCD corrections to massless identical quark scattering, Nucl. Phys. B 601 (2001) 341 [hep-ph/0011094] [INSPIRE].

[41] C. Anastasiou, E.W.N. Glover, C. Oleari and M.E. Tejeda-Yeomans, Two loop QCD corrections to massless quark gluon scattering, Nucl. Phys. B 605 (2001) 486 [hep-ph/0101304] [INSPIRE].

[42] E.I. Buchbinder and F. Cachazo, Two-loop amplitudes of gluons and octa-cuts in $N=4$ super Yang-Mills, JHEP 11 (2005) 036 [hep-th/0506126] [INSPIRE].

[43] F. Cachazo, Sharpening The Leading Singularity, arXiv:0803.1988 [INSPIRE].

[44] J. Gluza, K. Kajda and D.A. Kosower, Towards a Basis for Planar Two-Loop Integrals, Phys. Rev. D 83 (2011) 045012 [arXiv: 1009. 0472] [INSPIRE].

[45] R.M. Schabinger, A New Algorithm For The Generation Of Unitarity-Compatible Integration By Parts Relations, JHEP 01 (2012) 077 [arXiv:1111.4220] [INSPIRE].

[46] D.A. Kosower and K.J. Larsen, Maximal Unitarity at Two Loops, Phys. Rev. D 85 (2012) 045017 [arXiv: 1108.1180] [INSPIRE].

[47] S. Caron-Huot and K.J. Larsen, Uniqueness of two-loop master contours, JHEP 10 (2012) 026 [arXiv: 1205.0801] [INSPIRE].

[48] H. Johansson, D.A. Kosower and K.J. Larsen, Two-Loop Maximal Unitarity with External Masses, Phys. Rev. D 87 (2013) 025030 [arXiv:1208.1754] [INSPIRE].

[49] H. Johansson, D.A. Kosower and K.J. Larsen, Maximal Unitarity for the Four-Mass Double Box, Phys. Rev. D 89 (2014) 125010 [arXiv:1308.4632] [inSPIRE].

[50] M. Søgaard, Global Residues and Two-Loop Hepta-Cuts, JHEP 09 (2013) 116 [arXiv: 1306.1496] [INSPIRE].

[51] M. Søgaard and Y. Zhang, Multivariate Residues and Maximal Unitarity, JHEP 12 (2013) 008 [arXiv: 1310.6006] [INSPIRE].

[52] M. Sogaard and Y. Zhang, Unitarity Cuts of Integrals with Doubled Propagators, JHEP 07 (2014) 112 [arXiv: 1403.2463] [INSPIRE].

[53] K.J. Larsen, Global Poles of the Two-Loop Six-Point N=4 SYM integrand, Phys. Rev. D 86 (2012) 085032 [arXiv: 1205. 0297] [INSPIRE].

[54] S. Badger, H. Frellesvig and Y. Zhang, Hepta-Cuts of Two-Loop Scattering Amplitudes, JHEP 04 (2012) 055 [arXiv: 1202.2019] [INSPIRE].

[55] P. Mastrolia and G. Ossola, On the Integrand-Reduction Method for Two-Loop Scattering Amplitudes, JHEP 11 (2011) 014 [arXiv:1107.6041] [INSPIRE].

[56] S. Badger, H. Frellesvig and Y. Zhang, An Integrand Reconstruction Method for Three-Loop Amplitudes, JHEP 08 (2012) 065 [arXiv: 1207.2976] [INSPIRE].

[57] Y. Zhang, Integrand-Level Reduction of Loop Amplitudes by Computational Algebraic Geometry Methods, JHEP 09 (2012) 042 [arXiv:1205.5707] [INSPIRE]. 
[58] S. Badger, H. Frellesvig and Y. Zhang, A Two-Loop Five-Gluon Helicity Amplitude in QCD, JHEP 12 (2013) 045 [arXiv: 1310.1051] [INSPIRE].

[59] B. Feng and R. Huang, The classification of two-loop integrand basis in pure four-dimension, JHEP 02 (2013) 117 [arXiv: 1209.3747] [INSPIRE].

[60] P. Mastrolia, E. Mirabella, G. Ossola and T. Peraro, Scattering Amplitudes from Multivariate Polynomial Division, Phys. Lett. B 718 (2012) 173 [arXiv:1205.7087] [InSPIRE].

[61] P. Mastrolia, E. Mirabella, G. Ossola and T. Peraro, Integrand-Reduction for Two-Loop Scattering Amplitudes through Multivariate Polynomial Division, Phys. Rev. D 87 (2013) 085026 [arXiv: 1209.4319] [INSPIRE].

[62] P. Mastrolia, E. Mirabella, G. Ossola, T. Peraro and H. van Deurzen, The Integrand Reduction of One- and Two-Loop Scattering Amplitudes, PoS(LL2012) 028 [arXiv:1209.5678] [INSPIRE].

[63] R.H.P. Kleiss, I. Malamos, C.G. Papadopoulos and R. Verheyen, Counting to One: Reducibility of One- and Two-Loop Amplitudes at the Integrand Level, JHEP 12 (2012) 038 [arXiv: 1206.4180] [INSPIRE].

[64] R. Huang and Y. Zhang, On Genera of Curves from High-loop Generalized Unitarity Cuts, JHEP 04 (2013) 080 [arXiv: 1302.1023] [INSPIRE].

[65] P. Mastrolia, E. Mirabella, G. Ossola and T. Peraro, Multiloop Integrand Reduction for Dimensionally Regulated Amplitudes, Phys. Lett. B 727 (2013) 532 [arXiv:1307.5832] [INSPIRE].

[66] B. Feng, J. Zhen, R. Huang and K. Zhou, Integral Reduction by Unitarity Method for Two-loop Amplitudes: A Case Study, JHEP 06 (2014) 166 [arXiv:1401.6766] [INSPIRE].

[67] Z. Bern, J.J.M. Carrasco, H. Ita, H. Johansson and R. Roiban, On the Structure of Supersymmetric Sums in Multi-Loop Unitarity Cuts, Phys. Rev. D 80 (2009) 065029 [arXiv:0903.5348] [INSPIRE].

[68] M. Sogaard, Supersums for all supersymmetric amplitudes, Phys. Rev. D 84 (2011) 065011 [arXiv:1106.3785] [INSPIRE].

[69] F. Caola, J.M. Henn, K. Melnikov and V.A. Smirnov, Non-planar master integrals for the production of two off-shell vector bosons in collisions of massless partons, JHEP 09 (2014) 043 [arXiv: 1404.5590] [INSPIRE].

[70] A.V. Smirnov and V.A. Smirnov, FIRE4, LiteRed and accompanying tools to solve integration by parts relations, Comput. Phys. Commun. 184 (2013) 2820 [arXiv:1302.5885] [INSPIRE].

[71] P. Griffiths, J. Harris, Principles of Algebraic Geometry, Wiley-Interscience [John Wiley \& Sons], New York, 1978.

[72] R. Hartshorne, Algebraic Geometry, Springer-Verlag, New York, 1977, graduate texts in mathematics, no. 52 .

[73] D.R. Grayson and M.E. Stillman, Macaulay2, a software system for research in algebraic geometry, available at http://www.math.uiuc.edu/Macaulay2/.

[74] E. Cattani and A. Dickenstein, Introduction to residues and resultants: Solving polynomial equations, Springer Berlin Heidelberg, 2005.

[75] D.A. Cox, J. Little and Donal. O'Shea, Using algebraic geometry, Springer, New York, 2005. 